Portland State University

PDXScholar

$1-1-1975$

\title{
The relationship between the housing environment and the child's behavior: strategies for adapting to multi-family housing
}

Anthony J. Filipovitch

Portland State University

Follow this and additional works at: https://pdxscholar.library.pdx.edu/open_access_etds Let us know how access to this document benefits you.

\section{Recommended Citation}

Filipovitch, Anthony J., "The relationship between the housing environment and the child's behavior: strategies for adapting to multi-family housing" (1975). Dissertations and Theses. Paper 840. https://doi.org/10.15760/etd.840

This Dissertation is brought to you for free and open access. It has been accepted for inclusion in Dissertations and Theses by an authorized administrator of PDXScholar. Please contact us if we can make this document more accessible: pdxscholar@pdx.edu. 
THE RELATIONSHIP BETWEEN THE HOUSING ENVIRONMENT

AND THE CHILD'S BEHAVIOR: STRATEGIES FOR

ADAPTING TO MULTI-FAMILY HOUSING

by

ANTHONY J. FILIPOVITCH

A dissertation submitted to the Faculty of Urban Studies in partial

fulfillment of the requirements for the degree of

DOCTOR OF PHILOSOPHY

Portland State University

1975 
TO THE OFFICE OF GRADUATE STUDIES AND RESEARCH:

The members of the Committee approve the dissertation of Anthony J. Filipovitch presented July 30, 1975.
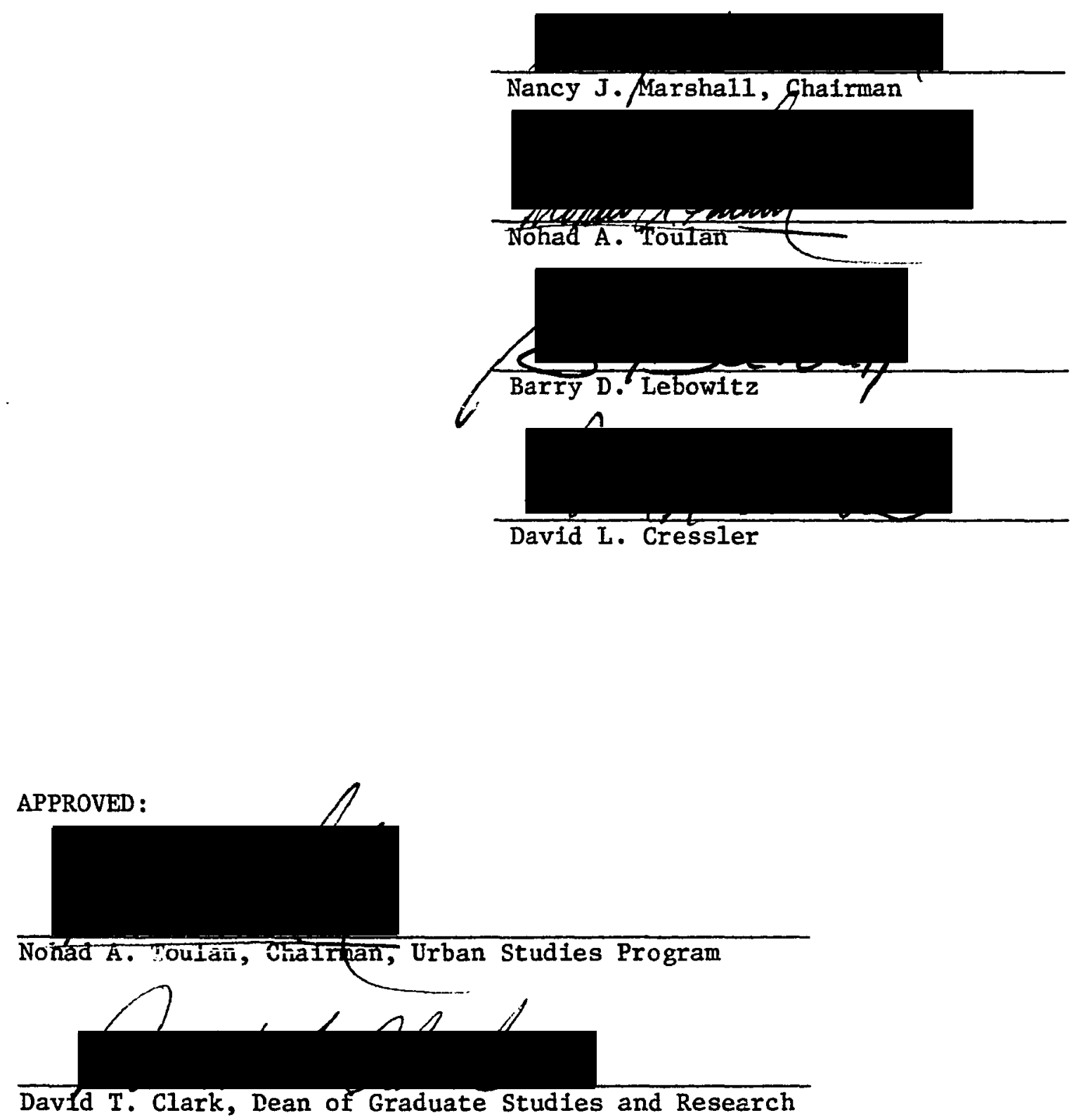

August 8, 1975 
AN ABSTRACT OF THE DISSERTATION OF Anthony J. Filipovitch for the Doctor of Philosophy in Urban Studies presented July 30, 1975.

TITLE: The Relationship Between the Housing Environment and the Child's Behavior: Strategies for Adapting to Multi-family Housing

APPROVED BY MEMBERS OF THE DISSERTATION COMMITTEE:

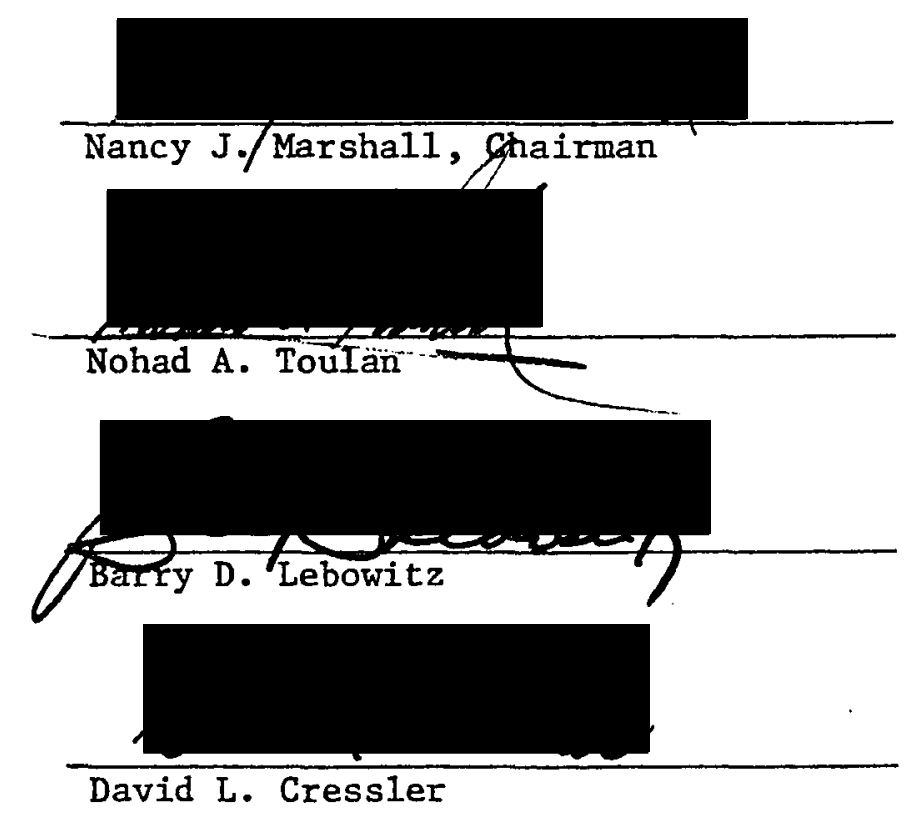

This study is an inquiry into some of the effects of the housing environment on the behavior of children. The city can be seen as a structure which facilitates communication and interaction because of the large number of intersecting individual pathways which the city represents. Young children, however, have little experience of this city: for the most part, their microcosm extends only a few blocks from home. Clties are a function of density and large numbers of pecple, and these two characteristics are aiso descriptive of multi-family structures. 
In the child's microcosm, multi-family dwellings could be considered as the type of communication-facilitating structure which is analogous to the city. The housing environment was examined along its physical dimension (housing environment as a piece of territory), socio-spatial dimension (social characteristics which are descriptive of the territory), and social dimensions (characteristics which are not tied to a specific spatial location). Children's behaviors were examined in terms of strategies for adapting to sensory load produced by the housing environment.

Three procedures were used to obtain data: observation of the children, at home and at a day-care center; interviews of both the child and a parent; and a test constructed by J.A. Desor to measure tolerance for social density. The subjects were 32 4- and 5-year-old children enrolled in the day-care center at Portland State University. Protocols of behavior episodes collected in field observation were coded by two coders, and inter-coder reliability was computed. The coefficient for a completeIy naive coder was 0.76 ; for a coder familiar with the concept of 'behavior episodes,' the coefficient was 0.86 .

Convergent validity of the behavioral measures was evaluated using a multitrait-nultimethod matrix. While the measures show high face validity, convergent validity was not established for similar measures drawn from the home and school environments. The exception to this was the measures of play-group size, which showed a consistent pattern of convergence. The matrix of correlations of measures which were descriptive of the housing environment showed a fairly clear clustering of the measures along the expected dimensions. A t-Test showed that there were significant differences between single-family and multi-family homes. 
In analyzing the hypotheses, it was found that children living in buildings with more peers show a greater acceptance of social density than children living in buildings with fewer peers. This strategy expressed itself in behavior also, for children living in buildings with more peers also played with more people. The relationship was crosssituational, suggesting that this strategy is a 'functional unity,' or a consistent pattern of the child's personality. It was also found that the number of people in the neighborhood was related to the extent to which the child rejected offers for interaction. However, since there were no significant differences in number of people in the neighborhood between single-family and multi-family dwellings, this relationship does not distinguish between apartment and single-family dwellers. It was also found that, if length of time in the present housing unit is controlled, there is a negative relationship between the number of people in the housing environment and the duration of play activity. No differences were found in the amount of solitary play or the number of settings used. 
To my parents, who were present at the creation. 


\section{ACKNOWLEDGEMENTS}

This study has been germinating for many years, its first seeds planted before I had even heard of Urban Studies or Portland State University. It bears a debt of gratitude to many people, too many to be mentioned here, many who probably are not even aware of what they wrought. My debt to them is paid by sharing with an open hand what they gave so freely.

Some people share the dubious honor of having helped me write this study. My Committee--Nancy Marsha1l, Nohad Toulan, Barry Lebowitz, and David Cressler--gave me support when I got discouraged, and gave me a kick when I got over-confident. I am grateful for the confidence they showed in me by serving as advisors, letting me work out my own problems. Credit should be given to John Hanson and John O'Brien for their damnable questions that kept me up nights; and to my friends and classmates, especially Colleen Greer Acres, Michael McCoy, Scott Klag, and Patrick Hampton for helping me answer them.

The research for this study was done while $I$ was the Dan Davis Fellow at Portland State. I am deeply grateful for that aid, not only for the time it freed, but for the freedom it gave me to follow ideas uninterrupted to their end.

Finally, I must acknowledge the kindness and cooperation of the children, their parents, and the staff at the Helen Gordon Child Development Center. Without their help, this study literally could not have been done. 
ACKNOWLEDGEMENTS ...................... . . . . . . . . .

LIST OF FIGURES AND TABLES . . . . . . . . . . . . . vii

CHAPTER

I. INTRODUCTION ................. 1

Definitions ................. 6

Limitations . . . . . = . . . . . 8

Organization of Study .......... 9

Notes ................ . . . 11

II. REVIEW OF THE LITERATURE 13

The Housing Environment and Residents' Desires 16

The Housing Environment and Activity Patterns 20

Density and Crowding .......... 26

The Child's Housing Environment . . . . 32

Notes ............... . . . 37

III. CONCEPTUAI, FRAMEWORK . . . . . . . . . . 44

Theoretical Background ........... 44

Specification of Key Variables . . . . . 58

The Hypotheses . . . . . . . . . 64

Notes ..................... 66

IV. METHODS .................. 70

Choice of Design . . . . . . . . . 70

Subjects ................. . . 75 
Procedures for Collecing Data . . . . . . 78

Operational Definitions . . . . . . . . 88

Procedures for Analyzing Data . . . . . . . 92

Notes . . . . . . . . . . . . . 105

V. RESULTS: ANALYSTS OF THE MEASURES . . . . . . . 108

Multitrait-Multimethod Matrix: Dependent Variables 108

Matrix of Correlations: Independent Variables 117

Summary Statistics: Dwelling-Type . . . . 120

Qualitative Analysis of Measures . . . . . 123

Summary : . . . . . . . . . . . . 128

Notes................. 130

VI. RESULTS: ANALYSIS OF HYPOTHESES . . . . . . . 131

Cognitive Structuring .......... 131

Withdrawal from Interaction . . . . . . 134

Filtering Potential Interaction . . . . . 137

Other Findings . . . . . . . . . . 146

Discussion . . . . . . . . . . 156

Notes............... . . . 162

VII. SUMMARY AND SUGGESTIONS FOR ADDITIONAL RESEARCH . • 163

Summary of Findings . . . . . . . . . 163

Suggestions for Additional Research . . . . 165

Notes . . . . . . . . . . . 172

REFERENCES ...................... . . . . . 173

APPENDICES . . . . . . . . . . . . . . . . 183 


\section{LIST OF FIGURES AND TABLES}

FIGURE

PAGE

1. Model of the effect of the housing environment on the child's behavior................

2. Schematic Design of a Multitrait-Multimethod Matrix.

TABLE

I. Matrix of Correlations: Dependent Variables ...

II. Matrix of Correlations: Independent Variables . .

III. Summary Statistics of Independent Variables by Dwelling Type . . . . . . . . . . . . . . . . . .

IV. Correlations: Cognitive Structuring and Communication Characteristics . . . . . . . . . . .

V. Correlations: Refusing Interaction and Communication Characteristics . . . . . . . . . . . .

VI. Correlations: Ignoring Interaction and Communication Characteristics . . . . . . . . . . . .

VII. Curve Fitting: Solitary Play and Communication Characteristics . . . . . . . . . . . .

VIII. Correlations: Duration and Communication Characteristics . . . . . . . . . . .

IX. Correlations: \% Peers in Play-Group and Communication Characteristics . . . . . . . . . . . .

X. Correlations: \% People in Play-Group and Communication Characteristics ... . . . . . . . . .

XI. Correlations: \# Peers in Play-Group and Communication Characteristics . . . . . . . . . . . .

XII. Correlations: \# People in Play-Group and Communication Characteristics . . . . . . . . . . . .

XIII. Correlations: \# Settings and Communication Characteristics . . . . . . . . . . . . .

XIV. Correlations: Cognitive Structuring and Control Variables . . . . . . . . . . . . . . . 
viit

TABLE

PAGE

XV. Regression Analysis: Cognitive Structuring . . .

XVI. Correlations: Dependent Measures and \% Life in Building Type ................

XVII. Correlations: Dependent Measures and Time in Building Type ............... .

XVIII. Correlations: Dependent Measures and Time in Building 152

XIX. Correlations: Dependent Measures and Time at School 153

XX. t-Test: Duration of Episodes for Single-Family and Multi-Family Children . . . . . . . . . .

XXI. t-Test: Differences in Dependent Measures Between Boys and Girls ...............

XXII. Chi-Square: Interaction Choices. All Choices .

XXIII. Chi-Square: Interaction Chosces. Minus Numbers . 
CHAPTER I

\section{INTRODUCTION}

It is too often forgotten in our brash, practical modern world that twilight, shadow, and beauty are as important to a growing child as food and air. 1

Marjorie Allen, Lady of the Hurtwood

There is a curious ambivalence in many Americans' attitudes about the City: they are both attracted and repelled by it. Many seem to feel that it's a nice place to visit, and even a convenient place to work, but they don't want to live there. It has not always been this way. For most of Western recorded history, the City has been regarded as a positive influence in the lives of men. Aristotle said that, "Men are animals who belong to a polis."2* At one time, the claim, "I am a citizen of Rome!"** was a claim to privilege and prestige anywhere in the (Western) civilized world. And any Medieval serf knew that, "City air makes men free. "***

But with the decline of the city-state (polis, civitas, Stadt), and the coming of the Industrial Revolution, this glowing image of the City tarnished. In the United States, a relative newcomer in matters of

*"Politikon ho anthropos zoon." This is more frequently translated, "Man is a political animal," but the word politikos--"political"-means "belonging to the polis (city-state)."

**"Cives Romani sum." The word cives--"citizen"--is derived from civitas, the word for city-state.

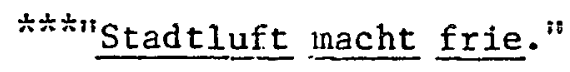


urbanization, the City has never been completely trusted. 3 The American mythos has emphasized the wholesomeness of the pastoral and feared the 'corrupting influence' of the City. The Big City was the means not only of Carrie's success but also of her loss on innocence in Dreiser's novel; Sister Carrie. 4 The development of this ambivalence in literature about the City has been traced out in detail in Leo Marx's study, The Machine in the Garden. 5

Increasingly, this ambivalence is developing into a conviction that cities are nasty places to live. When pressed to support this conviction, critics of the City give many reasons, such as air pollution, crime, and noise. A commonly expressed complaint is the unacceptability of city housing, especially multi-family structures. Dissatisfaction is expressed by city residents: "Whether the speakers were young parents or ancient grandads, they seemed to feel instinctively that multi-storey life was somehow alien to the children."6 It is voiced in the popular press:

Town planners and architects, too, come in for a good deal of blame [for rising crime rates] for creating skyscraper apartment blocks and vast, anonymous new towns where there is no space for children to play. 7

The charge is supported by Paul Friedberg, an urban designer:

...the urban environment has the power to desensitize the perceptions, cause an unnecessary physical strain, create a lingering disorientation, intensify a growing apathy and lack of involvement, limit the capacity to communicate with others, reduce the ability to learn and develop. 8

And it is supported by Holme \& Massie, social scientists:

Serious studies come up with different answers, but on balance, especially where young families are involved, the evidence suggests the view that this planners' expedient [high flats] has proved disadvantageous for the people who live there. 9

In other words, there is broad support, at all levels of opinion, that 
urban housing, especially apartment housing, is not a good place to live, especially for children. To return to an earlier quotation:

Whether the speakers were young parents or ancient grandads, they seemed to feel instinctively that multi-storey life was somehow alien to the children. So did most of the Glasgow doctors, psychologists and social workers consulted in this study, and the housing officials, architects and sociologists met with here and abroad. It was noted that the literature on the social aspects of multi-storey housing mostly concentrated on children and generally with misgivings. 10

But while there is all this conviction about the negative effects of the urban housing environment, especially on children, there is little hard evidence to support it. As early as 1951, Catherine Bauer had called for research on how people live and how the housing environment influences their behavior. 11 Although some work has been done in the area, twenty years later Jephcot could say: "Despite the furor, little hard statistical evidence has been obtained in this country or elsewhere about the effects of this new type of home on children...."12 Holme and Massie, cited earlier, can refer to only three studies, two of which "have not found as marked a difference as might be expected,"13 and the other showed simply that children in high flats don't play outside as much as children in detached dwellings. However, it is not shown in what sense it is "disadvantageous" for young children to play inside. Given our present knowledge, it appears that much of the conviction about the unsuitability of the urban housing environment, especially for raising children, is an assertion which, while perhaps not unfounded, finds little support in existing research.

This is not to say that urban-dwellers are not dissatisfied with their housing. Dissatisfaction is an attitude rather than an assertion which rests on empiricai verifiabilicy. Nor is it to say that the urban 
housing environment is not unacceptable to many people. Dissatisfaction with housing is enough to make it unacceptable. But it is to suggest that, if the reasons offered in support of the contention that urban housing is bad for children do not stand up to scrutiny, perhaps the dis:satisfaction expressed by that contention is misplaced and is more appropriately directed towards some other object. People may be dissatisfied not with apartment living per se, but with some of its concomitants (poor city planning, perhaps, or inadequate supply of public open space). Or perhaps the dissatisfaction occurs not because of the constraints apartment living puts on behavior, but because of the social significance of apartment 1iving. Chombart de Lauwe claimed that, "It is not possible to change the habitat and housing in any durable way except by changing the society and the family."14 And Cooper suggested that apartment structures will be given less status, given the tendency to attribute status in part on the basis of housing as a public presentation of an individual's tastes. 15 Perhaps the dissatisfaction expressed about apartment-dwelling could be due to culture lag: the social system may not yet have devised a way to satisfy the status and prestige needs of apartment-dwellers.

From another point of view, there is in fact no reason why urban housing and city living must be necessarily either good or bad. First, the city is neither a uniform nor a unidimensional environment. Not all of a city is apartment districts, and not all apartment districts are Midtown Manhattan. Second, people are neither uniform or unidimensional. Papanek scolds industrial designers for acting as if everyone fits the specifications of a World War II Dutch fighter-pilot ${ }^{16}$ (the group on which design specifications were originally standardized). Just as people 
are physical.1y distinguishable, they are also emotionally and intellectually distinguishable. Some people (like Jane Jacobs) really like New York City. And some people (1ike Carl Sandburg) really like Galesburg, Illinois. Space and landscape requirements cannot be universally specified because individuals differ in their expectations and cultures differ in the values which are ascribed to physical details, as Sonnenfeld has pointed out. 17

This study is an inquiry into some of the effects of the housing environment on children's behavior. It is a step in the direction of understanding how people experience the cities they live in and how they respond to that experience. It is not an attempt to evaluate either the urban environment or the urban experience; only after a phenomenon is understood may we then consider whether the ramifications of that phenomenom are desirable. Basic research into the effect of housing on behavior, represented by this study and others, is important in forming housing policy, in establishing a basis for some zoning law, 18 and in urban planning. A primary contribution of this study lies in the attempt to carefully explore some dimensions along which housing may have an effect, in order to better understand the dissatisfaction which people express. As Studer and Stea claim,

An environmental problem exists if and only if we can detect a malfunction between the designed environment and the system of human behaviors which is to be accommodated.19. 


\section{DEFINITIONS}

There are several key terms in this study that are commonly used in many different ways. The precise formulation of these terms will be done in Chapter III, but some preliminary discussion now will avoid confusion later.

The first of these terms is "housing environment." Not all urban housing is in apartment structures, and yet discussion of urban housing frequently reverts to discussion of apartment living. 20 In part, this is because apartment-living is typical of the city: as Fischer has pointed out, cities are a function of density and large numbers of people, 21 and these two characteristics are also descriptive of apartment structures (in comparison with single-family structures). Nor is all housing either single-family housing or high-rise apartment structures. There is a continuum from single-family homes on a one-acre wooded 1ot; to singlefamily homes on smaller lots; to duplexes and row houses; to town-houses and other two-story, four- or six-unit structures; to low-rise apartment structures of two or three stories; through high-rise apartment structures which are so tall that an elevator is a necessity. For the purposes of this study, the continum will be divided into two categories, single-family and multi-family dwellings located in urban areas (including the suburbs).

A second term that should be discussed is "behavior." Technically, behavior is "the observable activity of muscles and glands of external secretion."22 It is generally used in a broader sense, intending human activities which are in principle not directly observable, such as thoughts and attitudes. Any given behavior can be analyzed at many dif- 
ferent levels, from a consideration of small units of muscular activity (such as studies of pupillary reaction) to a consideration of patterns of much larger units of bodily activity (such as studies of courting behavior). Also, behavior is under conscious control to a greater or lesser degree. Most adults tie their shoes without thinking about each operation, or write a paragraph without focusing on the formation of each letter of each word. Breathing is usually not consciously controlled, yet breathing is responsive to the environment: in thinner air, the breath usually becomes shallower and quicker, automatically. All of these aspects of behavior will be touched on in this study, but the primary concern is with those behaviors that are not usually under conscious, formal control. People tend to grow accustomed to their environment, and in the process their response to the environment becomes habitual: no longer a focal, consciously controlled process, although conscious control could be exercised at any time.

Finally, the term, "children," should be discussed. The obvious distinction is that a 10-year-old is not a 1-year-old, nor is the difference between them 'ten times' as great (whatever that would mean). Piaget has shown that children at different ages perform in qualitatively different fashions--the differences are incommensurable. 23 It is important, then, to study children who are at roughly the same stage of development, unless one intends to consider developmental differences. The second distinction is that behavior is a function not only of developmental stages but also of prior experience. Not all children will have had the same variety (or range of varieties) of experiences with the housing environment. A similar point (in relation to adults) was made by Sonnenfeld in his discussion of native/nonnative differences in responding 
to the environment.24 Esser suggested also that some environmental effects may be due to cumulative experience, rather than immediate exposure. 25 The children used in this study were all pre-schoolers, ages 4 to 6. At this age, they are just beginning to play alone outside the house; thus, prior experience of a housing environment different from the present one is minimized, and the children as a group should be at roughly the same developmental stage.

\section{LIMITATIONS}

There are several limitations which had to be dealt with in designing this study.

The first limitation is the absence of unambiguous empirical knowledge in the area. As will be seen in the next chapter, there are no studies of children's housing environments which have used carefully controlled designs. Those studies that have been carefully designed have dealt with short-term effects of the immediate environment, usually a schoolroom. As a result, the present, state of the art is not supportive of large-scale carefully designed analysis. A first step must be to determine which aspects of which variables are most likely to yield productive data for a large-scale study. This limitation is discussed in greater detail in Chapter IV.

A second limitation has to do with the location of subjects. It is difficult to find a large, relatively homogeneous group of apartment children, perhaps because of the conviction of the unsuitability of apartments for childrearing. A sample could have been constructed from all over the city of Portland, but each additional apartment child would have differed from the rest of his group on so many other characteristics that 
an effect due to housing could conceivably have been suppressed by a massive within-group variance due to extreme heterogeneity. The alternative was to find a relatively homogeneous group with a large number of apartment-dwellers. This would have the advantage of highlighting any effect due to housing differences, but carries with it the limitation that the results cannot necessarily be generalized to a larger population. However, since the study is already limited to determining a potentially productive variable-space in which to carry out larger-scale studies, this limitation of generalizabiltiy was considered secondary to arriving at as clear an understanding as possible of the dimensions of such a variable-space.

A third limitation has to do with the choice of subjects. By limiting the study to one developmental stage, it is not possible to determine whether and how any effect continues its influence into adulthood. As children's home-ranges increase in size, does the effect of the housing environment decrease? Is there a critical stage, up to which the child 'imprints' on a home schema, and beyond which the housing environment has no effect? These and similar questions, necessary for a complete understanding of the effect of the housing environment on children's behavior, must await the clear and unambiguous determination of an effect at one developmental stage.

\section{ORGANIZATION OF THE STUDY}

The points raised in this introduction will be taken up and refined in the following chapters. These hapters are organized as follows:

Chapter II will review selected literature bearing on this study. The object is not to do an exhaustive review of all the extant literature, 
but to position this study within the research tradition.

Chapter III will bring the literature and the problem of this study into relation. It will develop the conceptual basis of this study, leading to a specification of the variables under consideration. Hypotheses will then be put forward.

Chapter IV will examine how the problem is best analyzed, given the current state of research. The procedure used in this study will be specified and the variables will be operationally defined.

Chapter V will consist of an analysis of the measures used in the study, examining their validity and their interrelationships.

Chapter VI will consist of an analysis of the hypotheses and other findings which relate to the hypotheses.

Chapter VII will consist of a summary and suggestions for future research. 
NOTES p. 11 .

1. Marjorie Allen, Planning for Play, Boston: MIT Press, 1969,

2. Aristoteles, Politics, Cambridge, Mass.: Harvard University Press, 1959, I, ii, 9 (1253 a).

3. Morton White \& Lucia White, The Intellectual Versus the City, New York: Mentor, 1962. 1900 .

4. Theodore Dreiser, Sister Carrie, New York: Doubleday, Page,

5. Leo Marx, The Machine in the Garden, New York: Oxford University Press, 1964.

6. Pearl Jephcot, Homes in High Flats, Edinburgh: Oliver \& Boyd, 1971, p. 80 .

7. Granville Wilson, "British blame rise in youth crime on greedy, indulgent, affluent parents," The Oregonian, September 29, 1974, p. 3-E.

8. M. Paul Friedberg, with Ellen Perry Berkeley, Play and Interplay, New York: Macmillan, 1970, p. 15.

9. Anathea Holme \& Peter Massie, Children's Play: A Study of Needs and Opportunities, London: Michael Joseph, Ltd., 1970, p. 73 ff.

10. Jephcot, 오. cit.

11. Catherine Bauer, "Social questions in housing and community planning," Journal of Social Issues, 1951, $7(1-2), 1-33$.

12. Jephcot, 오. cit., p. 81 .

13. Holme \& Massie, op. cit., p. 74.

14. Paul Chombart de Lauwe, Famille et Habitation, Tome I, Paris: Editions du Centre National de la Recherch Scientifique, 1959 (my translation). The French passage reads: "Changer I'habitat et la logement n'est possible d'une maniere durant qu'en changeant la societe et la famille," p. 11.

15. Clare Cooper, "The House as Symbol," Design and Environment, $1972,11,30-37$. 1971.

16. Victor Papanek, Design for the Real World, New York: Pantheon,

17. Joseph Sonnenfeld, "Variable values in space and landscape: An inquiry into the nature of environmental necessity," Journal of 
Social Issues, $1966,22(4), 71-82$. 1959.

18. Cf. Charles M. Haar, Land Use Planning, Boston: Little, Brown,

19. Raymond Studer \& David Stea, "Architectural programming and human behavior," Journal of Social Issues, 1966, 22(4), p. 131.

20. Cf. Elizabeth Coit, Report on Family Living in High Apartment Buildings, U.S. Government Printing Office, 1965.

21. Claude S. Fischer, "Urbanism as a Way of Life': A review and an agenda," Sociological Methods and Research, 1972, I, 187-242.

22. Donald 0. Hebb, A Textbook of Psychology, 2nd. Ed., Philade1phia: W.B. Saunders, 1966.

23. Jean Piaget \& Barbel Inheldex, The Child's Conception of Space, New York: Norton, 1967.

24. Sonnenfeld, op. cit.

25. Aristide H. Esser, "A biosocial perspective on crowding," in J.F. Wohlwill \& D.H. Carson, eds., Environment and the Social Sciences, Washington, D.C.: American Psychological Association, 1972. 
CHAPTER II

REVIEW OF THE LITERATURE

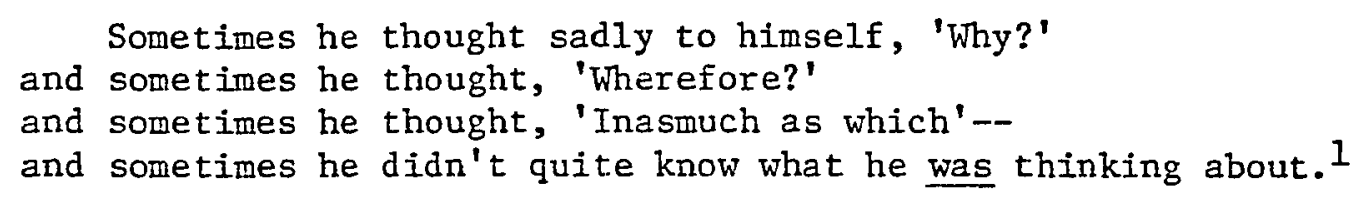

Eeyore

There is very little literature dealing directly with the effect of the housing environment on children's behavior. There is a larger body of literature dealing with the more general question of the effect of housing on the residents' behavior, although much of it is conflicting and fragmentary. These studies will be reviewed in order to better 1ocate this study within the research tradition.

Much of this literature seems to fall into two different camps. Some approach the housing environment as a natural phenomenon, something to be observed and analyzed in its own right. For such authors, the housing environment is a substructure which supports a given social system. As Chombart de Lauwe wrote:

To study the habitat... is to observe the image of society inscribed on the ground. To study the layout of a house is to analyze the relations between family life and the limits it imposes on itself or which society imposes on it.... It is not possible to change the habitat and housing in any durable way except by changing the society and the family. ${ }^{2}$

He went on to point out that, for the most part, it is more useful to consider the housing environment, society, and the family as a whole which is transformed simultaneously, rather than looking for unidirectional effects of one on the other. The weakness of this approach is 
that it can become a mere cataloguing of existing conditions and fail to consider the impact--functional and dysfunctional--of those conditions. Others approach the housing environment as it is dysfunctional for man's relation to the physical world. For such authors, 'housing' is the product of human activity, a product whose imperfections must be repaired. Paul Friedberg, the urban designer quoted in Chapter I, Iisted an assortment of ills due to the urban environment, and concluded that, "The environment batters us so devastatingly that no number of basketball games or picnics or bowling matches can neutralize the inpact." 3 From this point of view, housing is an independent variable which can be modified in order to transform the social environment. The weakness of this approach is that it can fail to consider the functional aspects of a (partially) dysfunctional physical situation, with the result that the 'cure' causes further disruption of the social environment.

The ideal, perhaps, would be a position midway between these two: to consider the housing environment as a social and physical ensemble which is more or less well-integrated among its parts. There would still remain, however, the question of whether change is accomplished by manipulation of discrete parts or by transformation of the ensemble--a question which will not be resolved here.

The task of understanding the effect of housing on the residents is further complicated, not only by the problem of finding a common perspective, but by the difficulty of clearly delineating the field of inquiry. The area is, of course, subject to all the pitfalls of research dealing with individual experience: perception is a selective process, and it cañ be difficult to ascertain just what an individual is perceiving; responses are filtered through the structure of the individual's per- 
sonality and past experience. As Parr put it:

Our task is admittedly complicated by the fact that the actual response to the environment is always a response to the total environment within the range of perception at the moment, and to all previous experience as well. Every moment of life is an expression of the entire life up to that moment. The total milieu is also more than the sum of its parts, both objectively and conceptually; and the perception of the environment involves all the senses at all times, either positively or negatively. 4

There are also, however, difficulties in delineating the inquiry that are specific to the problem-area in question. There is not, as yet, a commonly accepted use of the term 'housing environment.' As Gutman pointed out, some way must be found to deal with the housing environment as a physical structure and as a social network, preferably a way to deal with both aspects simultaneously. 5 A similar point was underlined by

\section{Thomas Fraser:}

Since man is the ultimate arbiter of any habitable state, the nature of his response to that state will act as a measure of its quality in terms of his physiological homeostasis, social relationships, and productivity. ${ }^{6}$

Fraser's statement also points to a second difficulty in delineating the effect of housing on residents: what is intended by the term 'response'? Parr pointed out that some responses may be emitted even if the individua? is unaware of them. ${ }^{7}$ Dean pointed out that in the category of 'needs' which individuals become aware of in their housing environment, some are clearly recognized explicit needs, some are taste preferences, and some are dimly apprehended wants. 8

Further, there is even a problem in categorizing the individuals who are responding. Sonnenfeld suggested that the significant dimensions of the response to the housing environment should be categorized, not in terms of culture, society, or economics, but rather in terms of whether 
the individual is a native or a nonnative. 9 He suggested that what really distinguishes individual responses to an environment is the individual's prior experience with different environments.

Finally, care must be taken in interpreting the significance of an individual's responses to his environment. Riemer pointed out that adjustment for dysfunctions in the housing environment can be accomplished either by changing the physical structure or by changing the individual's (and his social network's) attitudes.10 Sonnenfeld went further, suggesting that the adaptability of the individual can be presumed, and even demanded, necessitating less drastic adjustments in the physical characteristics of the housing environment. 11 This implies that the observable behavior of an individual may not indicate the total adjustment he has made in response to his environment. And, as Dubos pointed out, these hidden adjustments may have serious consequences:

Although man is highly adaptable and can therefore achieve adjustments to extremely undesirable conditions, such adjustments often have long-range, indirect effects that are deleterious. 12

\section{HOUSING ENVIRONMENT AND RESIDENT'S DESIRES}

The relationship between the housing environment and the desires of the residents (explicit needs, preferences, and dimly apprehended 'wants') appears to be on the order of Chombart de Lauwe's wholes ("ensembles"). For the most part, there seems to be a 'fit' between the residents and their environment. As we have seen, Sonnenfeld suggested that this is characteristic of 'native' residents: they are satisfied with where they are, in part because they are not familiar with anything else, in part because those who were not satisfied have left. Franke and Bartz, in a 
study of new housing projects in Germany, found that most people were satisfied with their environment--no matter which of the different housing types they sampled.13 This suggests that Wolpert's ecological model of migration ${ }^{14}$ is applicable: individuals move from one housing environment to another until the strain that results from mismatch of individual and habitat is reduced to acceptable levels.

The exception to the general 'fit' of housing environment and residents occurs in the case of substandard housing. Rosow pointed out:

...there is little evidence that satisfaction with new housing is directly related to livability resulting from design per se except when there is a significant improvement in housing, especially where people came from substandard housing....15

In other words, an increase in satisfaction with housing can be expected if the individual came from an unusually poor prior housing environment (and thus, is a nonnative to that environment). This position is supported by Chapin, who pointed to an improvement in housing satisfaction with a decrease in the number of people per room (and a concomitant increase in privacy); 16 and by Wilner and his colleagues, who noted a significantly greater degree of positive affect towards housing (compared to a control group) when slum-dwellers were movad to federal housing projects. 17 It is not known, however, whether this is a temporary or a permanent effect.

Also, the mere fact of substandard housing was not necessarily enough to evoke dissatisfaction. Hartman noted that the residents of Boston's West End expressed satisfaction with their housing, although they lived in apartments at high densities. He concluded that, "...for certain peopie the accepted standards of housing quality and quantity may be of secondary importance in determining residential satisfaction or 
dissatisfaction."18 In one particularly well-designed study, Chapin found no change in the morale of slum families who were rehoused in federal projects.19 Explanations for these differences point to the effect of social networks. Hartman suggested that, "the diversity and intensity of high-density living, which were so attractive in the West End, had little value in a situation where a sense of privacy, selectivity, and free choice were felt to be lacking."20 Yancey made a similar point in discussing the ill-fated Pruitt-Igoe project, suggesting that the working lower-class were less trustful and more dependent on social networks to establish friendships, and as a result the architectural space set aside for common use was not used, and became dangerous. 21

This recognition of social class differences in housing satisfaction and usage of physical living space leads to a second aspect of the relation between the housing environment and residents' desires, the aspect of cultural and subcultural styles in housing. The point is that, in a situation of reciprocal influence, the housing environment and the culture or subculture 'evolve' until each supports the other, until the housing environment is "the society inscribed on the ground." Thus, people want and expect a certain kind of housing environment which in turn reinforces the life-style of their culture. The hallmark work in this area is Rapoport's House Form and Culture, ${ }^{22}$ which attempted to establish just such a relationship in a cross-cultural perspective.

Willmott and Young identified a similar relationship by comparing a new estate outside London and an older working class neighborhood.23 They found that kinship ties were less important at the new estate, that the children moved away from their parents and set up independent households. They also found that the large houses at the new estate supported 
this situation, making it difficult for parents to live with their children (since the houses were not divided into apartments) or to live near them (since the houses were too large for just two people). The older neighborhood, however, was organized around kinship ties, and it also had a much greater diversity of building types which provided greater flexibility of living arrangements. Rosow also noted that there is evidence of important class differences in the meaning and valuation of privacy, that the lower-class place less emphasis on privacy than the middleclass. 24 Brower offered the suggestion that city dwellers. who lack private yard space, use the sidewalks for recreational space 25 --further reinforcing the high-density, public-life-style of urbanites.

This interrelationship of culture and the housing environment can be conceptualized, following Calhoun, 26 as a homeostatic system. Assuming that there is an optimal level of social stimulation for the species, a housing environment that encourages social contact must be countered by a culture that limits it; conversely, a culture that encourages social contact must be countered by a housing environment that filters or blocks out the overload. Eventually, a balance is reached between environmental limitations on social contact and cultural expectation of social contact. A suggestion that this is an appropriate conceptualization can be derived from Kiefer's study of a Japanese danchi. 27 In a situation where the housing environment was rapidly and greatly changed (the introduction of high-rise apartments), social organization was undermined. Kiefer was not, however, explicitly testing Calhoun's hypothesis, and the research is no more than a suggestion in this regard.

In summary, it can be seen that, generally speaking, the housing environment and the cultural milieu are fitted to each other and inter- 
penetrate each other. If individual residents find thenselves mismatched to their housing-cultural environment, they move until they find a congenial location. The exceptions to this general rule occur in times of radical change (in either the culture or the housing environment) and in situations where migration is not possible (i.e., the very poor, or where there are failures in housing market).

\section{HOUSING ENVIRONMENT AND ACTIVITY PATTERNS}

There is a considerably larger body of literature dealing with the relationship between the housing environment and observable residential behavior. An exceptionally large proportion is devoted to questions of density and crowding, and will be considered in the next section. The remaining literature, dealing with other aspects of the housing environment, will be considered according to Gutman's categorization of processes by which the housing environment influences behavior. 28 He pointed out that amenities in the housing environment can influence human survival; this process will be labeled "Health." Second, housing environments can facilitate or impede the use of sensory modalities, and thus can facilitate or impede transmission of information; this process will be labeled "Communication." Third, housing environments can interplay with the human capacity to endow objects with symbolic meaning; this process will be labeled "Social Symbolism." Finally, the housing environment can enable residents to see and visualize individual artifacts and organize them into spatial forms; this process will be labeled "Aesthetics."

\section{Health}

Wilner and his coileagues showed that improvement from slum housing results in decreased morbidity for children. 29 They found no significant 
effect for adults. Schorr, in his exhaustive review of studies of slum housing, concluded as well that there is substantial evidence linking poor housing and poor health. 30 He suggested that some of the relationship is due to the physical condition of the housing (peeling lead-base paint, structural and safety inadequacies); some is due to density (and the increased exposure to infection that comes with higher densities). Although he failed to mention it, some of the relationship is probably also due to the correlates of low income (less education, less medical and dental care, etc.).

There is also some suggestion in the literature that the housing environment can have some effect on the psychological health of its residents. The classic study is by Faris and Dunham, which showed that the distribution of psychopathologies is related to the ecological zones of the city. 31 Grootenboer offered the suggestion that high residential density (crowding) may be related to psychopathology. 32 Both of these studies, however, suffer the limitation that the causal linkages were not specified, and the effect may in the end turn out to be due to some other covariates of the housing environment.

There is a third fashion by which the housing environment has an effect on survival: through survival of the species. As Dubos said:

Because man is shaped by environmental forces, it is desirable that a wide range of experiences be made available to him so as to favor the phenocypic expression of various types of genetic potentialities. Diversity is an essential aspect of functionalism. 33

In other words, housing environments which supply only a limited range of stimulation will, over the long run, select against a diversified genetic pool. The end result would be a breed which is highly selected for a sin- 
gle environment--a sort of greenhouse flower which cannot survive in the wilds from which its forebearers came.

\section{Communication}

The process of communication can be considered from the viewpoint of the facilitation of sensory modalities or from the viewpoint of ensuing social contacts. The classic study on the relation of the housing environment to social contacts is the study by Festinger and his colleagues on the social ecology of group formation. 34 They found that the ecological basis of friendship was passive contacts which were determined by the required paths followed in entering or leaving one's home. The key variables here were physical and functional distances. As Keller pointed out, 35 and as Festinger, et a1., were ready to admit, this effect obtains only when the social aspects of the housing environment are relatively homogeneous, and even then the effect becomes weaker over time. Since the study was first published, it has been confirmed in other settings. Wells found that dividing large offices into smaller sections substantially changed the interaction patterns of the office.36 Blake and his colleagues found that soldiers bunked in cubicle arrangements made fewer but more intense acquaintances, mostly among their cubiclemates. 37 Jacobs pointed out, in a context broader than the house, that "passive contacts" are a function not only of one's own paths, but also of the paths of others. 38 Paths that have a high number of potential contacts attract others, thus further increasing the potential for passive contact. People attract people; eyes on the street promote safety which attracts more people. Smith summarized and generalized this: 
"It is the siting of domestic activities in a territory which patterns encounters between members of a household or between households in a neighborhood." 39

The housing environment can also be considered as it facilitates or obstructs the sensory modalities of perception. The key work in this area is Ha11's The Hidden Dimension, 40 where he discussed space as it presents itself to the senses, and the manner in which space can obstruct them. He pointed out, for instance, that a visual barrier is not necessarily an acoustical one. Smith took Hall's idea a step further, pointing out that barriers may be set up not only to sensory modalities but also to normative systems. As she put it, "The physical barriers which restrict [surveillance by outsiders] are thus boundaries to the scope or jurisdiction of norms identified with others than those present." 41

Another side to the relation between the housing environment, perception, and the social system is developed by Bechtel. He pointed out that the cultural definition of objects as "public" or "not-to-be-touched" can modify the relation between sensory facilitation and the housing environment:

The behavior settings available to city residents are nearly three times as many as those available to town residents. Yet the town residents have control over their settings while the city block residents are mostly onlookers or audience. Based on past research this means the city environment is largely over-manned and fosters passivity and apathy. 42

Plant pointed out that the too-great facilitation of perception that goes with large families living in few rooms can result in lack of self-sufficiency, destruction of lllusions, sexual maladjustment, negativism and irritability, and lack of objectivity.43 It was his contention 
that large families live in few rooms only if they are poor and have no choice. Wolpert pointed out that the usual response to a mismatch between the individual and his housing environment is for the individual to move to a new location, 44 presumably one where the facilitation of sensory modalities of perception is optimal, or at least more suitable.

Social Symbolism

Another process by which the housing environment influences activity patterns is through the symbolic value of the housing environment. According to Becker:

The concept of an environnental message, the way in which people interpret--consciously or unconsciously--the meaning of the physical environment as an indicator of the physical attitudes and intentions, values and status, of those who live in the environment or control its form is a useful way of trying to understand some of the behavioral consequences of environmental decisions. In situations where little verbal communication exists between groups, the physical environment can become the most viable communication medium. 45

The literature discusses the symbolic process of the housing environment in terms of identity and in terms of status.

One of the earliest indications of the value of the housing environment for identity was Fried and Gleicher's study of Boston's residents of the West End slum who were displaced by urban renewa1.46 They discovered a profound sense of dislocation and a feeling of loss for the old environment. Coit noted that a loss of identity frequently accompanies a move to high apartments, 47 apparently as a function of the separation from the ground and the absence of private territory. Parr suggested that "...identification depends upon a certain amount of physical distinction of the nook that is mine and the trail that leads to it."48 These points are summarized by Cooper: 
The high-rise apartment building is rejected by most Americans as a 'home' because it gives one no private territory on the ground, violates the archaic image of what a house is, and I would suggest, is perceived unconsciously as a threat to one's self-image as a separate and unique personality. 49

The value of housing for social status has been shown in studies of slum families who have moved to better housing. Both Chapin and Wilner, et al., report that such a change resulted in a significant increase in the residents' social status, household furnishings, social participation, and self-concept.50 Haar pointed out that the idea that housing has an identifiable character and status value has been enshrined in the Law as sufficient grounds for regulating what an individual can do with his property. 51

\section{Aesthetics}

The organization of spatial form has not been as extensively studied as the other three processes. The groundbreaking work has been done by Lynch who explored those characteristics of the environment that facilitate its imagability. 52 Lynch's work, however, deals with the entire urban environment rather than just the housing environment. On a smaller scale, Parr suggested that architectural ornamentation could serve a positive function as "compensation for the loss of sensory satisfaction."53 LeCompte pointed out that settings which appear adequate, even impressive, from an outsider's perspective, might be unsatisfactory from the inhabitants' point of view if it fails to involve them in it. 54 In fact, Pillorge criticized one study of the livability of a housing environment precisely for failing to consider this dimension.55 Brolin offered a process of designing housing environments which is focused primarily on drawing residents and their social needs into the design.56 
Surimary

It is a difficult task to sumarize these four processes, for the evidence is conflicting. On the one hand, Gutman has stated that:

Taken altogether, empirical studies do not make a very conpelling case for the argument that the site plan is an important influence on individual behavior and collective socialization. 57

On the other hand, Schorr has stated that:

Though the evidence is scattered, taken as a whole it is substantial. The type of housing occupied influences health, behavior and attitudes, particularly if the housing is desperately inadequate. 58

Neither of these men is without support for his position. Behind this conflict, as was pointed out in the beginning of this Chapter, is the lack of a common perspective, the lack of a universally accepted viewpoint. Gutman is speaking about site plans (a purely physical variable), and Schorr is concerned with housing (a variable with many social and psychological components).

\section{DENSITY AND CROWDING}

The difficulty in comparing the research on the relationship between the housing environment and the response of its residents lies, at least in part, in the confusion of terms. Even when differences between the physical and social environment of housing is considered, confusion frequently remains. A commonly occurring source for some of this confusion can be found in the understanding of "density."

Density--a large number of people per unit of space--is one of the three terms, along with heterogeneity and a large number of people, that Wirth considered sufficient to define an urban place.59 Simmel said that the psychological basis of the metropolitan type is the intensification 
of nervous stimulation, the "rapid crowding of changing images," which results from the urban environment. 60 Fischer pointed out that, of Wirth's three terms, density is the most crucial for social-psychological questions (like the effect of the housing environment on the residents' ' behavior).61 Nonetheless, there is no agreement or commonly accepted usage for the concept of density, or the related concept of crowding.

Almost fifteen years ago, Jane Jacobs pointed out that a distinction needed to be made between density (population per acre) and crowding (population per room).62 Park Avenue in New York City has sections of very high residential densities; Jacobs was suggesting that the people in those apartments probably are not crowded. Her suggestion was supported in the planning journals almost immediately. 63 There have been studies published since then, however, that fail to take this consideration into account .64

Other research has considered the differences between density and crowding to be a purely physical variable: the divisor is changed from acres to total floor area. Schmitt, comparing physical density and physical crowding measures, found that morbidity, mortality, and social breakdown were more closely related to density than crowding. 65 However, Winsborough reported that when other covariates were held constant the effect of density is less clear-cut;66 and an earlier study by Loring found no overail trend, either.67 Chapin reported that the ratio of persons per room("physical crowding") is significantly related to housing satisfaction; ${ }^{68}$ Hutt and Vaizey reported that physical crowding affects the nature and frequency of social encounters; 69 and Marshall suggested that there might be a relation between physical crowding and privacy preference, although the results were too scattered to lend strong support. 70 . 
Galle and his colleagues, comparing density and crowding, found that they were only moderately related, and found that physical crowding accounts for much of the variance in social pathology. 71 However, Carnahan and his colleagues, in a similar study with more statistical controls, found no effect. 72 Apparently, this formulation is not adequate, either.

As Lawrence pointed out, "crowding" is a social variable, dependent on the attitude of the individuals involved.73 This same point was made by Fraser when he pointed out that "man is the ultimate arbiter of habitable states,"74 and by Choldin who suggested that the significant dimension might be the number of interacting individuals rather than a body count. 75 Choldin went on to suggest the use of the terms "spatial density" for population per acre and "social density" for population per unit of floor space.

Several studies have employed this conceptualization. Freedman and his colleagues, studying the effect of density on cognitive tasks, varied both population and room sizes. They found no significant effect. 76 Griffit and Veitch, studying the effect of density on affective responses, influenced the Subjects' experience of the environment by varying the temperature. 77 They found a significant relation between negative affective responses and being hot and crowded. Bates, studying the effect of density on social interaction, maintained constant room size but varied the group sizes. He found a significant difference, varying by sex, in types of interaction. 78

The significance of the human meaning of a situation has been found in studies that are concerned with something other than social density, as well. Hartman pointed out that the socially dense West End was preferred to newer, more "adequate" housing because the residents had worked 
out a social structure that made the social density acceptable. 79 In other words, while socially dense, they were not crowded. A similar point was made by Mitchell when he pointed out that in Hong Kong doubling-up of occupancy results in strain when the residents are not related, but rarely is it experienced between relatives. 80 He also points out that quite high social densities are made more bearable when the parents permit their children to run free outside the home, easing the strain on space inside the home.

This research on the social determinants of crowding has resulted recently in a theoretical reformulation of crowding. One approach focuses on available space as the potentially manipulable variable. Briefly put, in this approach crowding occurs when the demand for space exceeds supply. Stokols has presented a systems-model of the variables at issue, pointing out that the experience of crowding is an interaction of personal and environmental factors. 81 He suggested that, "The limitations of space will engender an experience of crowding to the extent that it introduces noxious physical effects or places constraints on personal or social activities."82 Zlutnik and Altman divided the factors which determine crowding into three rather than two groups: the situation or environment (not just density, but also duration and richness); interpersonal determinants (control of interpersonal interaction); and psychological factors (past experience, subjective experience, expectations).83 The interaction of these three factors results in the demand. for more space. Although they recognized the possibility of coping strategies other than increased space, 84 and thus stand between Stokols' position and the one to be discussed next, the bulk of their discussion is concerned with the manipulation space. 
A second approach focuses on social stimulation as the potentially manipulable variable. Briefly put, in the approach "being crowded" means "receiving excessive stimulation from social sources." 85 Desor stated the implications of the two positions quite nicely:

In particular, the definitions of 'being crowded' differ for the two, and methods of alleviating it differ accordingly. If animals require a personal space, then 'being crowded' will be defined as 'not having the necessary amount of personal space' or 'having one's personal space invaded.' 'Crowded conditions' will be those in which individuals cannot maintain their respective personal spaces. If, on the other hand, animals require some level of stimulation from conspecifics, then 'being crowded' will be defined as 'reception of too much stimulation from conspecifics,' and 'crowded conditions' will be those in which individuals cannot sufficiently reduce the degree to which they perceive each other. If 'being crowded' is the former, then its reduction can be accomplished only by increasing available space or reducing the number of individuals--that is, by reducing density. If 'being crowded' is the latter, then its reduction can be affected by any method of reducing interindividual perception, of which decreasing density is only one. 86

Desor's work has found that the experience of being croweded is affected by the activity that is ongoing and by the architectural barriers present. Her work has been confirmed by Baum and Valins, who concluded that, "...a crowded environment may be characterized by large group sizes which result in unwanted social interaction and social overload.87 Esser also suggested that crowding is a result of sensory overload, and went on to point out that the pathological effects of crowding may be the result of cumulative crowding experience. 88

The difference between these two approaches is not as great as Desor suggested at first, for Stokols' modification of the personal-space position included the level of social stimulation as one of the determinants of the need for space, and Desor included the need for space as one of the buffers for social stimulation. In these milder forms, the difference between the two approaches is merely one of emphasis. 
An alternate to Desor's social-stimulation approach to crowding is Chalsa Loo's suggestion that crowding is the result of the structuring of stimulation. 89 She pointed out that density is the result of the interaction between the number of individuals (social density) and the amount of space (physical density). Thus, a very large room may not be very dense physically but may be socially dense because of the number of people it can comfortably hold. The experience of crowding is influenced by the degree of structure imposed on the density. The greater the structure, the less likely one is to feel crowded. Both Loo and Desor have identified architectural design and the activity involved as relevant dimensions of their models. The difference lies in whether the significant factor is the absolute level of stimulation or the ability to organize the stimuli. Perhaps Loo's model can be seen as a specification of Desor's.

These two models of crowding, which emphasize the effect of social stimulation, suggest that density (the physical state of numbers of individuals and the amount of space) and crowding (its affective or attitudinal component) can be considered as measures of the interaction potential of a place. In other words, the density of an area is a measure of the potential contacts an individual can make in that area, and crowding is a measure of the individual's responsiveness to that potential. Several of the studies which we have already examined support the hypothesis that the built environment has such an effect on interaction. The study by Festinger, et al., showed that functional distance (which is a function of the site-plan) influences the formation of friendships in marriedstudent housing. 90 Wells showed the same effect in a business office, and Blake, et al., showed it in an army barracks. 91 Smith pointed out that the siting of domestic activities patterns encounters between individuals, 
and Yancey pointed out that the failure to appropriately locate domestic activities had a part in the failure of Pruitt-Igoe.92 Keyfitz suggested that increased mobility has increased the effective density of the city by increasing the potential contacts.93 For a theoretical discussion of the relation between numbers of individuals and potential contacts, the reader is referred to Bossard for a mathematical discussion and Terrien and Mills for an organizational discussion. 94

\section{THE CHILD'S HOUSING ENVIRONMENT}

Much of the work dealing with the child's housing environment has focused on the play situation. The general consensus of the playground designers is that the needs of children have not been taken into account in the design of the city. Their reasons differ. Lady Marjorie Allen said :

The fact has to be faced that modern civilization interferes with a hard and heavy hand in the spontaneous play of children. The use made of land around buildings is still, almost always, totally unsuitable for children. Most of the vast rebuilding schemes in many countries are horrible places, planned without love and understanding. This arrogance, this paucity of innovation, this disregard of the worth and scale of the individual represents a world-wide disease and is one of the tragedies of affluence. The designer must devise the means for establishing a connection between the buildings he creates and the people on the ground. 95

Friedberg pointed out how difficult Lady Allen's prescriptions can be:

The environment is not the same for all age groups. The preschool child is taken by the hand on his limited travels near his home; his public environment is primarily the stoop, the sidewalk, the street, the little park. When he goes to school he has a whole new environment, perhaps five to ten blocks in diameter. The teenager's environment is broader still; the adult's is the entire city; and the elderly person's is again relatively restricted, his lessened mobility keeping him near home. 96 
Bengtsson offered specific design criteria:

In the ideal dwelling for a new family with children a boundary between indoors and outdoors hardly exists. The child moves freely from one to the other, enlarging the radius of its activities as its feelings of safety and selfreliance grow. As it investigates, the outside world increases the child's own experience. The escape route back to the mother, or her substitute, is always there, and this strenghtens the child's self-confidence....

The conclusion from all such investigations is the same. Living in a tall block has grave consequences for the family, and we must not shut our eyes to this. If high rise development is continued it will be necessary to accept the implications and arrange communal child supervision in an appropriate manner because this can, to a certain degree, help overcome the problem of contact between children. Lack of contact is a serious matter and can be the cause of neuroses, with unfortunate effects on the development of the personality. 97

Wade, rather than prescribing for children's play, attempted to delineate what children actually do on playgrounds.98 He found that, in free play, the patterns of activity are of short duration; elementary-school-aged children prefer moving to stable or realistic play equipment; girls use playground apparatus more; but he found no significant difference in patterns of play in relation to social class, sex, or type of apparatus used. Besides Wade's study of children's play on playgrounds, several studies have been made of children's spontaneous play around their home building, Winter found that apartment children were more supervised in their play--supervised indoors because of smaller dwelling size, supervised outdoors because of greater amounts of street traffic-and that they had fewer regular playmates than single-family children. 99 She found no differences in reported frequency of age-mate play. It should be noted that Winter's data was gathered from interviews of parents; no direct observation of the children was reported. Holme and Massie, in a review of several studies, came to the conclusion that high-density apartment living is disadvantageous for the children: the parents find it difficult to 
keep an eye on their children, and they are concerned about the safety of their children when the children are playing outside.100 The children, Holme and Massie contended, spend less time playing outside as a result. This conclusion was also reached by Lipman.101 But it is not clear how . Holme and Massie's own research supports their contention. They compared a planned, mediun-density pedestrian-segregated housing project (with apartments, some high-rise) with an old, unplanned, overcrowded and traffic congested neighborhood (with flats and row-houses). They found the apartment children playing at home, individually and "passively," while the children from the older neighborhood played away from home in groups, and more actively. They did not explain in what way either pattern is "disadvantageous." Further, White, in a study of a high-rise project in England, found a wide variety of play activity in the project.102 And on the basis of his observations in Hong Kong, Mitchell suggested that in situations of high residential density the parents will encourage the children to play outside, unsupervised, in order to ease the density in the home. These two studies raise doubts about Holme and Massie's conclusions.

There have also been other, more general studies of the relationship between the child's housing environment and his behavior. Merton pointed out that,

The dwelling unit is the locus of the initial socialization of the child: it is there that his character-structure is largely shaped. Not only are patterns of socialization typically enacted within the home; they appear in part to be oriented toward the house and its contents... 104

Parr further specifies what sort of shaping might occur: "...the more variable of two early environments produces an adult organism that is perceptually and behaviorally more alert, flexible, and able to cope with 
change."105 Field research has found more discrete, readily apparent effects. Winsborough, reviewing the studies of high population density, concluded that children are especially susceptible to illness and death as a result of dense housing environments. 106 Chombart de Lauwe found that the mothers reported that their children were more nervous (nerveux) since they moved into high-rise apartment projects. 107 Plant reported abnormal personality development as a concomitant of high residential density, 108 although his report was the result of clinical experience, was poorly controlled, and failed to consider the possibility of spurious results due, for instance, to the effect of poverty or social disorganization. In a more carefully designed study, Jephcot found that:

Despite the fears, little hard statistical evidence has been obtained in this country or elsewhere about the effects of this new type of home on children, nor has this Glasgow study added much. There were certainly no overt signs that their health suffered. 109

Stevenson reported that, "Although the data obtained were limited and tentative, they did indicate that the estate children were not lacking in things to do and places to go..."110 She noted the importance of daycare at the estate she studied: 65 percent of the families were either using day-care or planning to use it. She noted that apartment children obtained more pleasure from playing in the company of other children, rather than from playing with toys, possibly because toys were easily stolen when left laying around the relatively public grounds of the building. Dattner, on the other hand, attributed the lack of socializing which is typical of adult Parisians to the childhood training they receive as a consequence of living in high-density apartments.111

The difficulty with the field studies is that both the environments and the groups which were studied were so hetergeneous that no clear 
effect could be proven. There have been several more carefully controlled studies involving children at school. Hutt and Vaizey varied social density and found that, depending on personality differences, aggression increased as social density increased and total interaction decreased.112. Bates also varied social density in a day-care classroom, and found differences in social interaction which varied according to sex.113 Austin, studying the amount of ground covered by children both in classrooms and on the playground, found no relation between use of space and cognitive style, although she did find sex differences in the use of space.114 Loo found that high density resulted in more interrupted activity, less interaction, and less aggression.115 As Loo has pointed out elsewhere, 116 these studies of children are not comparable with most of the studies of the effect of density on adults, because the children were in unstructured free-play situations, whereas most studies of adults have used structured situations. Another difficulty with these studies is that they have all involved schoolroom situations, which may not be the sane class of environment as the housing environment. 
NOTES

p. 45 .

1. A.A. Milne, Winnie-the-Pooh, New York: E.P. Dutton, 1926,

2. Paul Chombart de Lauwe, Famille et Habitation, Tome I, Paris: Edition du Centre National de la Recherche Scientifique, 1959, p. 11 (my translation). The French text reads: "Etudier 1'habitat...c'est observer l'image de la societe les rapports entre la vie d'une famille et le cadre qu'elle a pu se donner ou que la societe lui impose.... Changer 1'habitat et la logement n'est possible d'une maniere durant qu'en changeant la societe et la famille."

3. M. Paul Friedberg, with Ellen Perry Berkeley, Play and InterPlay, New York: Macmillan, 1970.

4. A.E. Parr, "Environmental design and psychology," Landscape, $1964,14(2), 15$.

5. Robert Gutman, "Site planning and social behavior," Journal of Social Issues, $1966, \underline{22}(4), 104-105$.

6. Thomas M. Fraser, "Density, intensity, and stress," Ekistics, $1972, \underline{33}, 269$.

7. A.E. Parr, "Psychological aspects of urbanology," Journal of Social Issues, $1966, \underline{22}(4), 39-45$.

8. John P. Dean, "Housing design and family values," Land Economics, 1953, 29, 128-141.

9. Joseph Sonnenfeld, "Variable values in space and landscape: An inquiry into the nature of environmental necessity," Journal of Social Issues, 1966, 22(4), 71-82.

10. Svend Riemer, "Sociological theory of home adjustment," American Sociological Review, 194.3, ㅇ, 272-278.

11. Sonnenfeld, op. cit.

12. Rene Dubos, "Environmental determinants of human life," in D. Glass, ed., Environmental Influences, New York: Russell Sage, 1968.

13. J. Franke \& J. Bortz, "Contributions to the application of psychology in city planning, I," "Zeitschrift fur experimentelle und Angewandte Psychologie," 1972, 19, 76-78.

14. Julian Wolpert, "Migration as an adjustment to environmental stress," Journal of Social Issues, 1966, 22(4), 92-102.

15. I. Rosow, "The social effects of the physicai environment," Journal of the American Institute of Planners, 1961, 27, 127-133. 
16. F. Stuart Chapin, "The psychology of housing," Social Forces, $1951,30,11-15$.

17. Daniel M. Wilner, et al., The Housing Environment and Family Life, Baltimore: John Hopkins, 1962.

18. Chester W. Hartman, "Social values and housing orientation," Journal of Social Issues, 1963, 19(2), 130.

19. F. Stuart Chapin, "An experiment on the social effects of good housing," American Sociological Review, 1940, $\underline{5}, 868-879$.

20. Chester $W$. Hartman, "The limitations of public housing: relocation choices in a working-class community," Journal of the American Institute of Planners, 1963, 29, 294.

21. W.I. Yancey, "Architecture, interaction, and social control," in J. Wohlwill \& D. Carson, Environment and the Social Sciences: Perspectives and Applications, Washington; D.C.: American Psychological Association, 1972.

22. Amos Rapoport, House Form and Culture, Englewood Cliffs: Prentice-Hall, 1969.

23. Peter Willmott \& Michael Young, Family and Class in a London Suburb, London: Routledge \& Kegan Paul, 1960 .

24. I. Rosow, "The social effects of the physical environment," Journal of the American Institute of Planners, 1961, 27, 127-133.

25. Sidney Brower, "Streetfront and sidewa1k," Landscape Architecture, $1973,63(4), 364-369$.

26. John B. Calhoun, "The role of space in animal sociology," Journal of Social Issues, $1966, \underline{22}(4), 46-58$.

27. Christie W. Kiefer, "Personality and Social Change in a Japanese Danchi," Dissertation Abstracts, 1968, 29, 1246-B.

28. Gutman, op. cit.

29. Wilner, et al., op. cit.

30. Alvin L. Schorr, Slums and Social Insecurity, Washington, D.C.: Government Printing Office, 1963.

31. Robert E.L. Faris \& H. Warren Dunham, Mental Disorders in Urban Areas, Chicago: University of Chicago Press, 1939.

32. E.A. Grootenboer, "The relation of housing to behavior disorder," American Journal of Psychiatry, 1962, 119, 469-472.

33. Dubos, 오. cit. 
34. Leon Festinger, et a1., Social Pressure in Informal Groups, New York: Harper, 1950. 1968.

35. Suzanne Keller, The Urban Neighborhood, New York: Random House,

36. Brian W.P. Wells, "The psycho-social influence of building environment: Sociometric findings in large and small office spaces," Building Science, 1965, 1, 153-165.

37. Robert Blake, et al., "Housing architecture and social interaction," Sociometry, $195 \overline{6,19}, 133-139$.

38. Jane Jacobs, The Death and Life of Great American Cities, New York: Vintage, 1961.

39. Dorothy Smith, "Household space and family organization," Pacific Sociological Review, $1971,14,53-78$ (p. 56). 1966.

40. Edward T. Ha11, The Hidden Dimension, Garden City: Doubleday,

41. Smith, op. cit., p. 57.

42. Robert B. Bechtel, "A behavioral comparison of urban and small town environment," in J. Archer \& C. Eastman, eds., EDRA2,

Pittsburgh: Environmental Design and Research Association, 1970.

43. J.S. Plant, "Family living space and personality development," in N.W. Bell \& E.F. Vogel, eds., A Modern Introduction to the Family, New York: Macmillan, 1960.

44. Wolpert, 으. cit.

45. Franklin D. Becker, Design for Living: The Residents' View of Multi-Family Fousing, Ithaca, NY: Center for Urban Development, Corne11 University, May, 1974, p.3.

46. Marc Fried \& Peggy Gleicher, "Some sources of residential satisfaction in an urban slum," Journai of the American Institute of Planners, $1961,27,305-315$.

47. Elizabeth Coit, Report on Family Living in High Apartment Buildings, Washington, D.C.: U.S. Government Printing Office, 1965.

48. Parr, 1964, op. cit., p. 18.

49. Clare Cooper, "The house as symbol," Design and Environment, $1972, \underline{11}$, p. 32 .

50. Chapin, 1940, op.cit.; Wilner, et a1., op. cit.

51. Charles M. Haar, "Wayne Township: Zoning for whom?--In brief reply," Harvard Law Review, 1954, 67, 986. 
52. Kevin Lynch, The Image of the City, Cambridge: MIT Press, 1960.

53. Parr, 1964, op. cit., pp. $16 \mathrm{ff}$.

54. William F. LeCompte, "Behavior-settings as data-generating units for the environmental planner and architect," in J. Lang, et al., Designing for Human Behavior, Stroudsburg, $\mathrm{Pa}$ : Dowden, Hutchinson, \& Ross, Inc., 1974.

55. George Pillorge, "Intensity of Development and Livability of Multi-Family Housing Projects: A review," Journal of the American Institute of Planners, 1964, 30, 257-259.

56. B. Brolin, "Mass housing: Social research and design," Architectural Forum, 1968, 129, 66-71.

57. Gutman, ㅇp. cit., p. 31 .

58. Schorr, ㅇp. cit., p. 31 .

59. Louis Wirth, "Urbanism as a way of 1ife," American Journal of Sociology, 1938, 44, 1-24.

60. Georg Simmel, "The Metropolis and Mental Life" in The Sociology of Georg Simmel, Kurt Wolff, ed., Glencoe: Free Press, 1950.

61. Claude S. Fischer, "Urbanism as a Way of Life': A review and an agenda," Sociologica1 Methods and Research, 1972, 1 , 187-242.

62. Jacobs, op. cit.

63. J. Marshall Miller, "Residential density: Relating to space rather than to ground area," Journal of the American Institute of Planners, $1961,27,77-78$.

64. Calhoun, op. cit., and "Space and the strategy of life," in A. Esser, ed., Behavior and Environment, New York: Plenum, 1971; George M. Carstairs, "Overcrowding and human aggression," in H.D. Graham \& T.R. Curr, eds., Violence in America: Historical and Comparative Perspectives, Washington, D.C.: U.S. Government Printing Office, 1969; Halsey M. Marsden, "Crowding and animal behavior," in J.F. Wohlwil1 \& D.H. Carson, eds., Environment and the Social Sciences: Perspectives and Applications, Washington, D.C.: American Psychological Association, 1972.

65. Robert C. Schmitt, "Density, health, and social disorganization," Journal of the American Institute of Planners, $1966,32,38-40$.

66. Halliman $H$. Winsborough, "The social consequences of high population density," Law and Contemporary Problems, 1965, 30, 120-126.

67. William C. Loring, Jr., "Housing characteristics and social disorganization," Social Problems, 1956, 3 , 160-168. 
68. Chapin, 1951, op. cit.

69. C. Hutt \& M. Vaizey, "Differential effects of group density on social behavior," Nature, 1966, 209, 1371-1372.

70. Nancy C. Marsha11, Orientations Toward Privacy: Environmental. and Personality Components, unpublished Ph.D. dissertation, University of California at Berkeley, 1969.

71. 0.R. Galle, et al., "Population, density, and pathology: What are the relations for man?" Science, 1972, 23-30.

72. Douglas Carnahan, et al., "Urbanization, population density, and overcrowding: Trends in the quality of life in urban America," Social Forces, 1974, 53, 62-72.

73. John E.S. Lawrence, "Science and sentiment: Overview of research on crowding and human behavior," Psychological Bulletin, 1974, $\underline{81}, 712-720$.

74. Fraser, op. cit., p. 269.

75. Harvey M. Choldin, "Population density and social relations," Paper presented at a meeting of the Population Association of America, Toronto, April 14, 1972.

76. Jonathan Freedman, et a1., "The effect of crowding on human task performance," Journal of App1ied Social Psychology, 1971, 1, 7-25.

77. William Griffit \& Russe11 Veitch, "Hot and crowded: Influences of population density and temperature on interpersonal affective behavior," Journal of Personality and Social Psychology, 1971, 17, 92-98.

78. Brian C. Bates, Effects of Social Density on the Behavior of Nursery School Children, unpublished Ph.D. dissertation, University of Oregon, 1970 .

79. Hartman, op. cit.

80. Robert E. Mitchell, "Some social implications of high density housing," American Sociological Review, 1971, 36, 18-29.

81. Daniel Stokols, "A social-psychological model of human crowding phenomena," Journal of the American Institute of Planners, 1972, 38, 72-83.

82. Ibid., p. 82 .

83. Steven Zlutnik \& Irwin Altman, "Crowding and human behavior," in J.F. Wohlwill \& D.H. Carson, eds., Environment and the Soclal Sciences, Washington, D.C.: American Psychological Association, 1972.

84. Ibid., p. 54 . 
85. Jeanette A. Desor, "Toward a psychological theory of crowding," Journal of Personality and Social Psychology, 1972, 21, 79-83, p. 79.

86. Jeanette A. Desor, The Psychology of Crowding: An Experimental Investigation, unpublished Ph.D. dissertation, Cornell University, 1969, p. 20 .

87. Andrew Baum \& Stuart Valins, "Architecture, social interaction, and crowding," Transactions of the New York Academy of Science, 1974, 36, 793-799, p. 793 .

88. Aristide H. Esser, "A biosocial perspective on crowding," in J.F. Wohlwill \& D.H. Carson, eds., Environment and the Social Sciences, Washington, D.C.: American Psychological Association, 1972.

89. Chalsa Loo, "Important issues in researching the effects of crowding on humans," Representative Research in Social Psychology, 1973, 4, 219-226.

90. Festinger, et al., op. cit.

91. Wells, op. cit.; Blake, et al., op. cit.

92. Smith, op. cit.; Yancey, op. cit.

93. Nathan Keyfitz, "Population density and style of social life," Bioscience, $1966, \underline{16}, 868-873$.

94. James H.S. Bossard, "The law of family interaction," American Journal of Sociology, 1945, 50, 292-294: Frederic W. Terrien \& Donald L. Mills, "The effects of changing size upon the internal structure of organizations," American Sociological Review, 1955, 20, 11-13. p. 11 .

95. Marjorie Allen, Planning for Play, Boston: MIT Press, 1969,

96. Friedberg, op. cit., p. 16.

97. Arvid Bengtsson, Environmental Planning for Children's Play, New York: Praeger, 1970, p. 13.

98. Glenn R. Wade, A Study of Free Play Patterns of Elementary School Age Children on Playground Equipment, unpublished Master's Thesis, The Pennsylvania State University, 1968.

99. Mary Winter, Apartment and Single Family Dwellings as They Affect the Play Activities of Pre-School Children, unpublished Master's Thesis, The Pennsylvania State University, 1966.

100. Anathea Holme \& Peter Massie, Children's Play: A Study of Needs and Opportunities, London: Michael Joseph, Ltd., 1970. $2-6$.

101. Marvin Lipman, "Housing and Environment," Habitat, 1969, 12(2), 
102. L.E. White, "The outdoor play of children living in flats," in L. Kuper, ed., Living in Towns, London: Cresset, 1953.

103. Mitche11, op cit.

104. Robert K. Merton, "The Social Psychology of Housing," in Wayne Dennis, ed., Current Trends in Social Psychology, Pittsburgh: University of Pittsburgh Press, 1948.

105. Parr, 1966, op. cit., p. 42.

106. Winsborough, o․ cit.

107. Paul Chombart de Lauwe, Famille et Habitation, Tome 2, Paris: Centre National de la Recherche Scientifique, 1960.

108. J.S. Plant, "Family living space and personality development," in N.W. Bell \& E.F. Vogel, eds., A Modern Introduction to the Family, New York: Macmillan, 1960. 1971.

109. Pearl Jephcot, Homes in High Flats, Edinburgh: Oliver \& Boyd,

110. Anne Stevenson, et al., High Living: A Study of Family Life in Flats, Melbourne: Melbourne University Press, 1967. 1969.

111. Richard Dattner, Design for Play, New York: Van Nostrand,

112. Hutt \& Vaizey, 으. cit.

113. Bates, op. cit.

114. Martha C. Austin, "Relationship between play area and cognitive style in pre-school children," Child Study Journal, 1974, 3, 51-67.

115. Chalsa Loo, "Effects of Spatial Density on Social Behavior of Children," Dissertation Abstracts, 1971, 32, 4189-B.

116. Loo, 1973, op. cit. 


\section{CHAPTER III}

CONCEPTUAL FRAIEWORK

Dr. Pell was wont to say that in the Solution of Questions, the Maine Matter was the well-stating of them; wch requires mother-witt \& Logick...; for let the question be but wellstated, it will worke almost of itselfe.1

John Aubrey

\section{THEORETICAL BACKGROUND}

The reconceptualization of urban density in terms of a measure of potential contacts, whether it is considered as the level of social stimulation or as the structuring of stimulation, offers what appears to be a promising approach to the problem of understanding at least some of the effects of the housing environment on its residents. As we have seen in the previous chapter, other approaches have yielded contradictory evidence, in part because of the lack of agreement on conceptualizations of the environment. On the other hand, this approach, which was first suggested by Simmel, 2 has not yet been extensively tested, although the early studies are promising. ${ }^{3}$ Further, it has the advantage of dealing with urban housing in terms of one of the major characteristics of urban life; density, ${ }^{4}$ while clarifying the relationship between density and housing. The earliest statement of this approach to urban living in terms of its potential for contact was Simmel's "The Metropolis and Mental Life."5 Simmel formulated the problem on a more general level, of which social contact is a special case. He contended that the typical urban type of personality is the result of the intensification of nervous stimulation that is a concomitant of the urban environment. As a result of this bom- 
bardment, the urban type develops behaviors which permit him to survive in such an environment--he accomodates himself to the city. This accommodation, Simmel suggested, takes the form of an increased intellectualization or depersonalization of activity. Urban types are blase, they keep each other at an arm's length, they are indifferent to each other, they exaggerate their differences. Simmel used the example of the plethora of stimuli that strike anyone who is just standing on the corner in a city. It is not entirely clear from his analysis what the role of face-to-face contact is in the urban overstimulation. It is apparent that social contacts are at least part of the stimulation, although non-personal contacts are also pointed to.

Around this same time, Burgess developed his concentric-zone hypothesis of the structure of the city. ${ }^{6}$ His purpose was to show that there was an interrelationship between the physical and social form of the city, and that this relationship was mediated by the demographic form of the city. His concern is in many ways similar to the concern of this study, but he focused on the effect of demographic-ecological variables on social variables, positing a unidirectional effect. Research done since then, some of which was considered in the previous chapter, has shown that the effect of either population per acre or population per unit of floor area (both demographic variables) has no simple or unmediated relationship to social structure: the effect is complex, probably the result of the interaction of several variables. At least the assumption of unidirectionality must be relaxed. Second, this study is concerned with the effect of the housing environment on individual behavior, rather than its effect on social groups. The significance for this study of Burgess' work is that the core of the city represents the maximum possible density of inter- 
secting pathways. Just as all diameters of a circle intersect in the center, so do journeys through the city tend to converge on the core.

The next real advance along these lines came with Lewis Mumford.7 Mumford stressed that the complexity of the city, Simmel's "intensification of nervous stimulation," is also the reason that cities exist. For Mumford, the city serves as the repository for Civilization. It must be arranged so as to have the maximum of facilities for storing the products of civilization in the least amount of space in order to encourage the transmission of those products. At the same time it must be flexible enough to contain a variety of different products and be able to enlarge enough to have a place for newer products. Although he was concerned with the physical products of civilization in this part of his analysis, ${ }^{8}$ he expanded it later to include the social products of civilization: "The translation of ideas into common habits and customs, of personal choices and designs into urban structures, is one of the prime functions of the city." 9 In other words, the city is a place where maximal storage and transmission of ideas and choices occurs. This can be seen as a synthesis of Burgess' realization that the core of the city represents the maximization of intersecting pathways and Simmel's realization that the individual responds to the complexity of the city (the maximation of paths), to which Mumford adds that this complexity, this intensity of stimulation, is actively sought by the individual, that it is the very reason for the existence of the city.

This conception of the city as a place for the transmission and storage of ideas was examined in some detail by Richard Meier. ${ }^{10}$ He examined the city as a structure for maintaining a high frequency of contacts. He pointed out that communication can be considered as a substi- 
tute for space or time in many urban activities. He contended that the attractiveness of a city is determined by the frequency of contacts, and that the usual measures used in determining attraction--distance and cost of movement--are surrogates for the first measure, which is more difficult to obtain. This relation between the attractiveness of a place and distance was explained by Carrothers in his analysis of gravity and potential models. Il The import of Meier's analysis for this study is that, as a structure for maximizing contacts, the urban environment must provide for both a high frequency of potential communications and a means to protect urbanites from the arrival of repetitious, redundant, and trivial communications. It should be noted that Meier was concerned only with communication, in the sense of exchange of information. He does not deal with the fact that contacts involve more than interchange of information, but are interactions and that what may be trivial in terms of exchange of information ("Good morning. Nice day, isn't it?") may be a very necessary function of human contacts. In part, this is because Meier had focused on the city in terms of the system of institutions rather than in terms of the individuals who are part of the institutions.

Milgram continued this line of thinking and applied it to the behavior of individuals.12 He pointed out, following Simmel, that the city represents a high level of stimulation. The experience of this stimulation is one of overload, and of adaptation to this overload. Milgram identified six strategies for adapting to it: 1) less time may be allocated to each input; 2) low priority inputs may be disregarded; 3) the burden of interaction can be shifted to the other party; 4) reception of stimuli can be blocked prior to input; 5) intensity of overload can be diminished by filtering; and 6) specialized institutions can be developed 
to deal with overload.

In summary, the city can be seen as a structure which facilitates communication and interaction because of the large number of intersecting individual pathways which the city represents. That is, the intersecting pathways facilitate contacts between people and thus represent potentials for interaction. This potential for interaction is experienced as overstimulation or overload, and necessitates adaptive strategies for dealing with it. These strategies can be embodied in the structure of the city (Meier) or in the individual (Milgram). Following Chombart de Lauwe, it could be expected that eventually there would evolve a fittedness between the urban structure and the residents there. This study is an examination of the relationships between these adaptive strategies and children's housing environments.

\section{The Housing Environment and Children's Behavior}

While the city can be seen as facilitating communication and interaction because of the large number of intersecting pathways which it represents, young children have little direct experience of an entire city: their microcosm usually extends only a few blocks from home. For a preschool-age child, the world revolves around the family dwelling unit. At first, he is never out of sight of it. In time, the child's range may expand to include a whole block, or several blocks if there are no busy streets between them. In such a microcosm, where the home range is the family dwelling unit and its imediate environs, multi-family dwellings could be considered as the type of communication-facilitating structure analogous to the city.

Compared to single-family dwellings, multi-family dwellings repre- 
sent family units which are unbuffered from each other. An apartment building is a more dense milieu, in terms of the absolute number of people in the building. The greater number of people in the building offers the possibility of a greater number of different contacts, more differences • in the individuals contacted, and the probability that public settings will be overmanned. In other words, the greater number of people increases the potential number of intersecting pathways. This is particularly true in the case of those multi-family structures that have a common inside hallway or staircase. In this case, the door to the building serves as a funnel for the individual paths, greatly increasing the probability of intersecting paths (or, as Festinger, et a1., would put it, 13 greatly decreasing the functional distance between individuals). Nor is the increase in potential contacts only in the public spaces of the housing milieu. Although multi-family dwellings do not necessarily imply crowding in Stokols' sense of the demand for space exceeding the supply, the interior arrangement of spaces in multi-family dwellings is frequently more compact. There are generally fewer rooms per unit, or smaller rooms per unit, and they are Erequently arranged in a single plane (i.e., there are few two-story apartment units). All of these characteristics of multi-family dwellings relative to single-family dwellings can be seen to facilitate greater numbers of contacts and interactions.

There are, of course, more ways to categorize a housing environment than just the communication configuration of the housing structure (single- or multi-family dwellings). The size of a dwelling unit can be expected to vary commonly from one bedroom to four bedrooms. The number of people inhabiting any given-sized dwelling can also be expected to vary from less than one person per room to almost two people per room. The 
physical quality of any given type of structure can be expected to vary from dilapidated to brand new. And even the communication-configuration of structures need not be considered as a simple dichotomy. Housing structures can be categorized on a scale from rural estates, to suburban developments, to single-family detached dwellings on small city lots, to row houses and duplexes, to townhouses and small apartments, to low-rise apartment structures, to high-rise apartment towers. It is obvious that the communication configuration of a housing structure is only one component of the housing environment; it is the component on which this study Is focused.

\section{Discrimination in Responding to Stimuli}

To this point, the discussion of the theoretical framework has dealt specifically with the potential for conmunication and interaction that is represented by the housing environment. The reasons for that qualification w111 now be discussed.

Simply put, the problem is that perception is a selective process. Responses are filtered through the structure of the individual's personality and past experience. In Parr's words, "Every moment of life is an expression of the entire life up to that moment."14 There are two implications to this. The first, as Zlutnik and Altman pointed out, 15 is that the experience of crowding is a function not only of the physical and interpersonal situation in which the individual finds himself, but it is also a function of the past experience of the individual. The second implication follows from this. The requirements for physical space and for human contact ("social space," as it were) cannot be universally specified because people adapt dffferently to spatial situations; and because 
within any given strategy for adapting, different values can be ascribed to the same empirical situation. 16 These points are summarized by Lowenthal:

Yet in daily practice, we all subordinate reality to the world we perceive, experience, and act in. We respond to and affect the environment not directly but through the medium of a personally apprehended milieu. The milieu differs for each of us according to his personal history, and for each of us it varies also with mood, with purpose, with attentiveness. What we see, what we study, and the way we shape and build the landscape is selected and structured for each of us by custom, culture, desire, and faith. To understand perceptual processes requires examination of all these facets of human behavior.17

That this process of selection is ubiquitous and multi-dimensional has been shown experimentally. Kaplan and his colleagues studied the relationship between rated preference and complexity for natural and urban visual material. They found that there is a definite preference for the more complex material; that there is also a preference for natural visual material (pastoral scenes); and that the natural visual material was not rated as more complex than the built environment.18 In other words, preference was found to be a function of both the complexity of the material and the ascribed value of the material. Robbins attempted to apply to urban areas the cross-cultural studies that have shown a relation between the perceptual environment one is used to and preference for patterns which are different from what one is usually exposed to. He found no relation, but suggested that the media was able to counterbalance the expected differences in pattern preferences between large cities and small towns. 19 In other words, he posited an effect due to prior experience. In other directions, both Wines and Maslow and Mintz studied the effect of the surroundings or the situation in which the perception is occurring. Wines pointed out that architectural design is perceived differantly 
by pedestrians and motorists, due to the angle at which the design is presented, the speed at which it moves by, and due to the angle of vision (the automobile-window frames the view and limits the angle of vision).20 Maslow and Mintz found that the aesthetic characteristics of a room could influence the subjects' rating of photographs of faces. 21 Piaget and Inhelder have shown that the response to space (and, by inference, the perception of space) is a developmental process which proceeds by recognizable stages. 22 Laurendeau and Pinard have confirmed those findings in empirical studies. 23

The point underlying all of these dimensions of selectivity is that discrimination occurs in regard to what is not perceived. This same point was made by Natsoulas: "How things look (and why) needs to be distinguished from the fact that things do look or appear." 24 A similar point was made by Hebb when he distinguished between perception, which is a process, and a percept, which is a result of the process. 25 In other words, between the stimulation of the sensory organs and an awareness of the world, there is a process of interpreting or construing the sensation. In discussing the relation between geography and a lived-world, Lowenthal used the term "appreciation" for this--the perceiving of what is not directly given in the sensation.26 When presented with a ba11, I "perceive" it to be spherical although this is not directly given in the sensory input: the side I cannot see could be pushed in or completely absent. Yet, because in the past whenever a ball was presented it turned out to spherical, I perceive this ball to be a sphere and respond to it in that expectation. In other words, what is perceived is the end result of a discriminative process of giving meaning to a raw sensory input; as Beck put it, "Indeed, meaning and perception are iriseparable."27 And Beck further 
pointed out that the meaning given in perception is at least in part a function of individual and developmental factors:

The spatial field is differentially charged with meaning from individual to individual; and particular configurations of the spatial field may be important clues to personality.... As these spatial styles become more and more a part of the personality structure of the individuals, space is slowly divided into definitive zones and directions with intuitive meanings and an expressive character of their own. 28

Although the importance of this question of apperception has been thoroughly explored in phenomenological philosophy, 29 it is not unknown to social science. It lies behind the Gestaltists' concern with good form. 30 It is implied in Hull's analysis of reaction potential $\left({ }_{s} E_{r}\right)$ and the forces which influence it. 31 It is supported by Hebb's theory of redounding circuits in the brain which are needed to organize perception. 32 And it is specifically discussed as an issue for cognition by Jerome Bruner under the rubric of "going beyond the information given."33 In fact, it was precisely the realization that perception is a selective process that led Woodworth to modify the traditional S-R (Stimulus-Response) formula into S-O-R (Stimulus-Organism-Response). 34

In summary, this study deals with the housing environment as it represents a potential for comminication and interaction because the housing environment constitutes a stimulus-field which must first be perceived before it can have an effect. There is a firm tradition in the social sciences which is aware of the fact that perceiving is, at least in part, a selective and an individually determined process. This means that an identity between the potential for contact and the actually perceived contacts cannot be assumed, but is instead an empirical question to be researched. 
Implications of the Choice of Children

There are several substantive considerations which have led to focusing this study on the effects of the housing environment on the behavior of young children.

In the first place, there have been suggestions in the literature, and even some research which supports the suggestion, that early experiences of the environment weigh heavily on later adult responses. While few go as far as Sommer who referred to this effect "imprinting,"35 several theorists have noted the effect. Dubos suggested that environmental effects are "...most profound and lasting--indeed often irreversible-when they take place early in life, during the developmental periods..."36 Parr, quoted earlier, suggests that the variability of the early environment has a formative influence on later adult cognitive style. 37 And Sonnenfeld's native/nonnative distinction 38 clearly implies some sort of formative influence of prior, if not early, experience. There has also been some empirical support for this position. Schooler found that childhood experience of the housing environment (measured in terms of its complexity) has a broader effect on adult psychological functioning than do variables such as adult social class.39 Witkin and his colleagues found that, while the absolute level of measures of cognitive style changed with age, the relative level was quite stable through adulthood. 40 Perhaps the explanation for such an effect would be that the first schema one develops for the housing environment serves as the yardstick against which all later environmental experience (including experience of the same environment at a later age, when one's needs are different) is measured.

Second, aside from the question of the continuing effect of early environmental experience, there is also some evidence that young children 
are more sensitive to any potential effect of the immediate environment. This has already been discussed in terms of the child's 'microcosm.' The young child has a smaller range and so is more tied to the immediate environs of his dwelling. This suggests that, if the dwelling-unit and its environs are to have an effect on the residents, $1 t^{\circ}$ would be most likely to show up in those residents who are most tied to it. Friedberg pointed out that the choice is then between the very young and the very old.41 If prior experience is a confounding factor, the very old would be a poorer choice. Also, Klausner cited evidence which suggests that "the human ecological pattern is more affected by a physical feature when the culture is less developed."42 While this is not intended to be a return to Heinz Werner's theory that "primitive people" are developmentally at the same level as "civilized children,"43 this particular case appears to be one in which the analogy is.appropriate. The child's command of the culture's techniques for modifying the impact of physical factors is certainly less developed than the adult's (e.g., a child cannot get in his car and drive to the country, or even to the park), and so we could anticipate that the child would be more reactive to the immediate physical environment than the adult, if Klausner's interpretation of the evidence is correct.

There are also reasons of application which led to the choice of young children as Subjects. Merton pointed out that the house is the locus of initial socialization. 44 Any negative effect of housing would have more serious consequences at this stage than later, when there would be an initial socialization which the child could draw on to deal with whatever problems the environment raises. Further, the older the child the more the socialization process is over-determined, at least as far as any ef- 
fect housing could have. Second, both Esser and Parr have pointed out that many of the effects of the housing environment are due to cumulative experience with the environment. 45 By choosing young children, the cumulative effects can at least be minimized in order to obtain something like a baseline measure from which to evaluate responses at later ages.

Behavior and Behavior Episodes

Finally, there are specific problems which are raised by the concern with "behavior," problems that bear on the conceptualization of behavior. In the first place, behavior may be a response to a situation of which the individual is not consciously aware. This issue was considered in the last chapter, where it was pointed out that responses may be emitted even if the individual is unaware of them, 46 and that 'needs' for housing vary from specific demands to dimly apprehended wishes to needs that are not considered by the residents at all but are supplied by the designer. 47 The implication is that sone effects of the housing environment may be nonfocal and unattended; the pressure of the environment might result in unmediated responses in some situations.

In considering behavior, then, it is not enough to determine what the residents are aware that they are doing. In fact, there is good reason to attempt to study behaviors that they do not focus on, that they are not aware of. When the residents are aware of their behavior, they can attempt to exercise conscious control of it should the behavior not accord with their cultural norms for such behavior. Unattended behavior, on the other hand, permits an environmental response unmediated (or at least less mediated) by social constraints. With this in mind, the focus of this study will be on behaviors which are not usually the object of 
awareness: the duration of an activity, the number of people and the number of places involved in play activity.

There is a second problem, which is the delimitation of the units of behavior. There must be some reasonably consistent fashion of deciding what is the unit of behavior, and when it begins and ends. Fortunately, the groundwork has already been done by Barker and Wright. 48 According to them, a 'behavior episode' is the smallest coherent behavioral entity in the behavior stream, and is determined by three attributes: constancy of direction, normal behavior perspective, and equal potency. These characteristics of behavior episodes will be examined in greater detail in the following chapter.

The use of Barker and Wright's behavior episodes carries with it, however, the problem of determining the setting in which the behavior is occurring, for a change of setting is frequently a clue to a change of behavior episode. LeCompte, a student of Barker and Wright, summarized the definition of a behavior setting as "...one or more extra-individual patterns of behavior and milieu, with the milieu being circumjacent to and synomorphic with the behavior pattern."49 In other words, a behavior setting is a recurring pattern of behavior-in-a-location, where the location encloses the activity and supports it. This is a useful definition for extensive field-studies.

But this study is concerned with very few settings: the home and its immediate surroundings, and a day-care center (or rather, the classrooms inside the day-care center). Most of the interest will be in microsettings which occur inside the larger setting (home or day-care center). The difference lies in the scope of analysis. Barker and Wright used the more complete name of standing behavior settings, or the technical refer- 
ence, K-21 behavior settings (from the formula used to determine whether a given setting met the defining criteria). They were concerned with recurring patterns of fittedness between behavior and environment.

In this study, the analysis is much more fine. As will be discussed in the next chapter, the "normal perspective" is measured in terms of seconds rather than minutes. Thus, while the settings will be circumjacent and synomorphic with the behavior, they will not not necessarily be part of a standing pattern. They will indicate simply the intensity, the fineness of discrimination of environmental detail, with which the child is involved in his environment. Since Barker and Wright always use the phrase "behavior setting," these smaller units, these micro-settings, will be referred to as simply "settings," to acknowledge the debt to Barker and Wright and yet to distinguish between the two concepts.

\section{SPECIFICATION OF KEY VARIABLES}

Housing Environment

It has already been shown that, among the components of a housing environment, the facilitation of communication and interaction is a useful component for consideration. The dimensions of this component must still be laid out.

Lee suggested that the physical dimension of the housing environment (housing environment as a piece of territory) should be distinguished from the socio-spatial dimension (social charateristics which are desciptive of the territory), and both should be distinguished from the social dimension (characteristics wich are not tied to a specific spatial location. 50 The idea here is that the physical characteristics of the environment are a different order of event than those social characteristics 
which are tied to a specific physical location. This was seen, for instance, in Loo's distinction between spatial and social density:51 a room of 100 square feet with ten people in it is experienced differently than a room of 50 square feet with five people in. it (holding spatial density constant), and differently than a room of 50 square feet with ten people in it (holding social density constant). The amount of free space available is a different order of event than the total number of contacts one is exposed to in a given setting.

This study focuses on the behavioral effect of single-family and multi-family dwellings, emphasizing the physical (dwelling-type, dwellingsize) and socio-spatial (social density) dimensions of the housing environment (i.e., those dimensions that are spatially bound). The social dimensions (family size, social class) will not be examined in detail in this study.

Communication Facilitation

The conception of "facilitation of communication and interaction" must be further specified.

This concept was introduced earlier through the work of Mumford and Meier. 52 They did not distinguish between "contact potential" and "interaction potential." In terms of adult behavior, this is probably an adequate formulation. In the case of children, however, it may be the case that there is a class of contacts that are not potential interactions. There is the possibility that children do not perceive adults (or older children) as suitable subjects with whom to initiate interaction. A similar suggestion was made by Harvey $\operatorname{Cox}$ in the case of contacts between adults of different statuses 53 ("you don't carry on meaningful interaction 
with the parking-lot attendant"). This possibility of strictly functional contacts has been examined in depth by Lofland.54 If children do limit their interactions with adults, then a distinction must be made in this study between total potential contacts (which include adults), and potential Interactions (which would be limited to children). For the purposes of this study of young children, a peer is anyone ten years of age or younger; an 'adult' (i.e., a 'non-peer') is anyone over ten years of age.

\section{Behavior}

Finally, the conception of a child's behavior in the housing environment must be specified. Milgram suggested that the city, by reason of its high level of communication-facilitation, is a stressful situation due to the resultant sensory overload.55 Following the reasoning that multifamily dwellings are analogous to the city in the case of young children, young children living in multi-family dwellings would be expected to develop strategies for dealing with sensory overload, strategies which should be less common in children from single-family dwellings.

Milgram divided the strategies for adapting to sensory overload into six categories. The six categories are: allocation of less time to each input; selection of inputs to disregard low priority ones; shifting the burden of interactions to the other party; blocking reception prior to input; diminishing the intensity of overload by filtering; developing specialized institutions to deal with overload. For the purposes of this study, the six can be synthesized into three categories: Withdrawal from interaction; Filtering of potential interactions; and Cognitive structuring.

The first five of Milgram's strategies are concerned with adapting 
to the stress of sensory overload by modifying the sensory input. Both "selection of inputs to disregard low priority ones" and "blocking reception prior to input" entail cutting off the possibility of stimulation. In the first, interaction is cut off by Ignoring it; Milgram used the example of unlisted telephone numbers. These strategies can be summarized as "Withdrawal from interaction." Children can withdraw from interaction by playing alone or by rejecting other children's overtures for interaction.

The remaining three strategies, "allocation of less time to each input," "shifting the burden of interaction to the other party," and "diminishing the intensity of overload by filtering," entail permitting only part of the total stimulation to come into play. Limiting the time devoted to any activity also limits the opportunity for any activity to absorb all of the individual's attention. Shifting the burden of interaction permits the individual to deal with only part of the current situation, that part of it which is put forward by the other party. One need not examine the situation for possible initiations, but can be content to react to the other's initiations. Milgram used the example of bus drivers who used to make change for the passengers; now the passenger has to have the correct fare. Filtering, the interposition of screening devices between individuals, permits weaker and relatively superficial forms of involvement. Milgram used the example of a mayor's office: in a small town, anyone can stop in for a chat with the mayor; in the large cities, the citizen is shunted off to one or another bureau. These strategies can be summarized as "Filtering potential interactions"--rather than cutting off the possibility of stimulation, the filtering strategies admit some of the stimulation, but in manageable doses. 
Milgram's sixth strategy, "developing specialized institutions," appears to be concerned with adapting to the stress of sensory overload by modifying the framework within which the sensations are perceived. This strategy entails the transformation of sensory overloads where the sensations cannot be controlled. Milgram used the example of welfare departments, which serve to control what would otherwise by "an army of mendicants continuously importuning the pedestrian."56 Milgram is speaking here of an adaptation by the entire social system to deal with stress due to overload. In this study, the concern is with the individual rather than the group, and with a type of individual that lives in a microcosm of the city, the young child. Milgram's sixth strategy suggests that the (social) framework can be organized so that impinging stimuli do not constitute an overloading of any part of that framework; by analogy, for a young child this strategy could be seen as the organization of the conceptual framework so that impinging stimuli do not constitute an overloading of any conceptual schema. This study is concerned with the schema for social density. This strategy will be referred to as "Cognitive structuring," and it means that children can adapt to stress due to overload by structuring their cognitive schemata in such a way that the sensation no longer constitutes an overload of any schema.

This third strategy conflicts with the first two strategies: if the overload can be satisfactorily modified, presumably there would be no need to modify the conceptual framework; and conversely, if the conceptual framework has been modified to fit with the level of sensory input there would be no need to modify sensory inputs. These strategies are not, however, logically contradictory. The absence of input modification does not necessarily entail modification of the conceptual framework: the 
possibility of a third alternative has not been ruled out. Further, it is not necessarily the case that any single strategy is sufficient to cope with the totality of sensory overload. It is conceivable that stress due to overload could be reduced primarily with one strategy (e.g., cognitive structuring) while the remaining stress is handled with another strategy (e.g., filtering potential interactions). Thus, this study examined all three strategies in turn, and confinmation or rejection of the use of any single strategy is not to be taken as confirming or denying the use of any other strategy.

The Model

This conceptual framework can be summarized in a model (Figure 1). This model represents the housing environment in its three dimensions, and the relationship of these dimensions to the child's behavior. The model presents the physical dimension of the housing environment as having a direct effect on the socio-spatial dimension. Since this study is not focally concerned with the social dimension of the housing environment, it does not attempt to present the interrelationships of the social dimension with the other dimensions. It presents all three dimensions of the housing environment as having direct but not necessarily indentical effects on the child's behavior in two different environments, one of which is the housing environment itself and the other is the day-care setting. From this model and the conceptual discussion, hypotheses can be derived. 


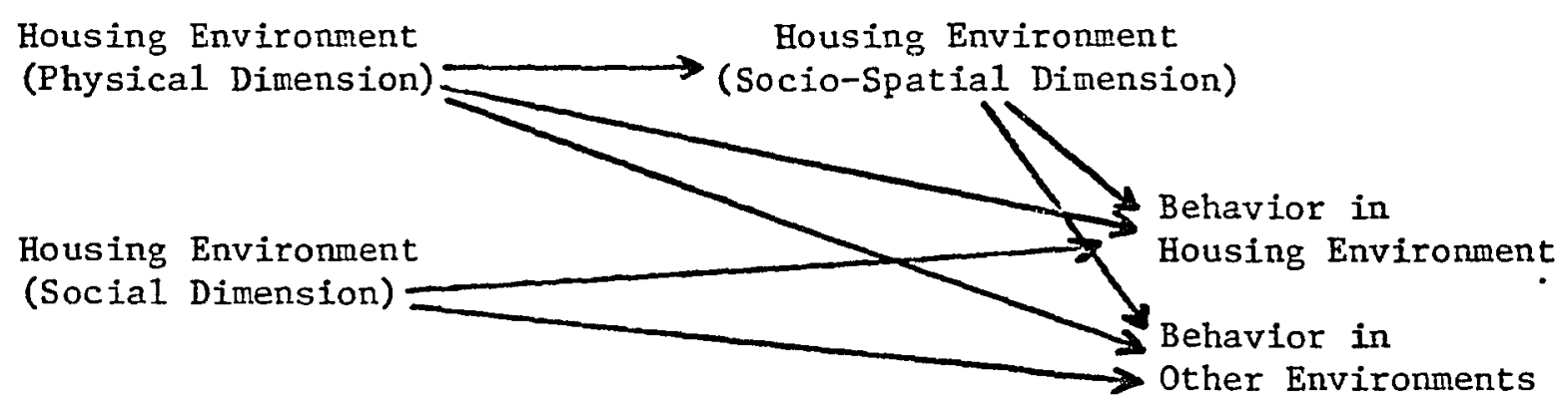

Figure 1. Model of the effect of the housing environment on the child's behavior.

III. HYPOTHESES

The hypotheses are concerned with relation between the socio-spatial-dimension of the housing environment and the child's behavior.

The first hypothesis examines the relation between the contactfacilitating characteristics of the housing environment and modification of the conceptual framework, measured by tolerance for social density. Contact-facilitating characteristics (the number of people) are used because social density is presumed to be as likely to be influenced by oneway contacts (i.e., an adult giving a command to a child) as well as by reciprocal interactions.

1) Children living in housing environments with greater numbers of people show greater acceptance of social density than children living in housing environments with fewer people.

The other two sets of hypotheses examine the relation between the interaction potential of the housing environment and modification of sensory inputs. Since the strategies deal with interaction, measures of interaction-potential (the number of peers) are called for. The second set of hypotheses deals with the strategy of withdrawal from interaction.

2a) Children living in housing envirouments with a greater number 
of peers reject overtures for interaction more frequently than children living in housing environments with fewer peers.

2b) The relationship between the number of peers in the housing environment and solitary play is described by an inverted-U function: at both very low and very high numbers of peers, solitary play occurs more frequently.

The third set of hypotheses deals with a strategy of filtering potential interactions.

3a) Children living in housing environments with a greater number of peers allocate less time to each behavior episode than children living in housing environments with fewer peers.

3b) Children living in housing environments with a greater number of peers interact with a smaller proportion of the potential playmates in the environment than children living in housing environments with fewer peers.

3c) Children living in housing environments with a greater number of peers use fewer settings than children living in housing environments with fewer peers. 
NOTES

1. John Aubrey, Brief Lives, Oliver Lawson Dick, ed., Ann Arbor: University of Michigan Press, 1957.

2. Georg Simnel, "The metropolis and mental 1ife," in The Sociology of Georg Simme1, Kurt Wolff, ed., Glencoe: Free Press, 1950.

3. Jeanette A. Desor, "Toward a psychological theory of crowding," Journal of Personality and Social Psychology, 1972, 21, 79-83; Andrew Baum \& Stuart Valins, "Architecture, social interaction, and crowding," Transactions of the New York Academy of Science, 1974, 36, 793-799.

4. Louis Wirth, "Urbanism as a way of 1ife," American Journal of Sociology, 1938, 44, 1-24.

5. Simnel, op. cit.

6. Ernest W. Burgess, "The growth of the city: An introduction to a research project," in R.E. Park, E.W. Burgess, \& R.D. McKenzie, The City, Chicago: University of Chicago Press, 1925.

7. Lewis Mumford, The City in History, New York: Harcourt, Brace $\&$ World, 1961.

8. Ibid., p. 30 .

9. Ibid., p. 113 .

10. Richard A. Meier, A Communication Theory of Urban Growth, Cambridge: MIT Press, 1962.

11. Gerald A.P. Carrothers, "An historical review of the gravity and potential concepts of human interaction," Journal of the American Institute of Planners, 1956, 22, 94-102.

12. Stanley Milgram, "The experience of living in cities," Science, $1970,167,1461-1468$.

13. Leon Festinger, et al., Social Pressure in Informal Groups, New York: Harper, 1950.

14. A.E. Parr, "Environmental design and psychology," Landscape, $1964,14(2), 15-18$, p. 15 .

15. Steven Zlutnik \& Irwin Altman, "Crowding and human behavior," in J.F. Wohlwill \& D.H. Carson, eds., Environment and the Social Sciences Washington, D.C.: American Psychological Association, 1972.

16. Joseph Sonnenfeld, "Variable values in space and landscape: an inquiry into the nature of environmental necessity," Journal of Social 
Issues, $1966, \underline{22}(4), 71-82$.

17. David Lowenthal, "Introduction: Environmental perception and behavior," in D. Lowentha1, ed., Environmental Perception and Behavior, Chicago: University of Chicago, Department of Geography, Research Paper 109,1967, p. 1 .

18. Stephen Kaplan, et a1., "Rated preference and complexity for natural and urban visual material," Perception and Psychophysics, 1972, $12(4), 354-356$.

19. Michae1 C. Robbins, "Perceptual environment and pattern preferences," Perceptual and Motor Skills, 1968, 26, 545-546.

20. James Wines, "Notes from a passing car: the problem of art in a mobile environment," Architectural Forum, 1973, 139(2), 66-75.

21. A.H. Maslow \& N.L. Mintz, "Effects of aesthetic surroundings, I," Journal of Psychology, 1956, 41, 247-254.

22. Jean Piaget \& Barbel Inhelder, The Child's Conception of Space, New York: Norton, 1967.

23. Monique Laurendeau \& Adrien Pinard, The Development of the Concept of Space in the Child, New York: International Universities Press, 1970 .

24. Thomas Natsoulas, "The subjective, experiential element in perception," Psychological Bulletin, 1974, 81, 611-631, p. 613.

25. Donald 0. Hebb, "Concerning Imagery," Psychological Review, $1968, \underline{75}, 466-477$.

26. David Lowentha1, "Geography, experience, and imagination: Toward a geographical epistemology," Annals of the Association of American Geographers, 1961 , 51, 241-260.

27. Robert Beck, "Spatial meaning and the properties of the environment," in D. Lowenthal, ed., Environmental Perception and Behavior, Chicago: University of Chicago, Department of Geography, Research Paper 109, 1967, p. 21.

28. Ibid., p. 19.

29. Edmund Husserl, Cartesian Meditations, tr. Dorion Cairns, The Hague: Martinus Nijhoff, 1960; Maurice Merleau-Ponty, The Phenomenology of Perception, London: Routledge \& Kegan Paul, 1962.

30. Wolfgang Kohler, Gestalt Psychology, New York: Liveright, 1929.

31. Clark L. Hull, Principles of Behavior, New York: AppletonCentury-Crofts, 1943. 

1949.

32. Donald 0. Hebb, The Organization of Behavior, New York: Wiley,

33. Jerome S. Bruner, Beyond the Information Given, New York: Norton, 1973.

34. Robert S. Woodworth, Psychology, Rev. Ed., New York: Ho1t, Rinehart, \& Winston, 1929.

35. Robert Sommer, "Man's proximate environment," Journal of Soctal Issues, $1966,22(4), 59-70$.

36. Rene Dubos, "Environmental determinants of human life," in $D$. Glass, ed., Environmental Influences, New York: Russell Sage, 1968.

37. A.E. Parr, "Psychological aspects of urbanology," Journal of Social Issues, $1966,22(4), 39-45$, p. 42.

38. Sonnenfeld, op. cit.

39. Carmi Schooler, "Social antecedents of adult psychological functioning," American Journal of Sociology, 1972, 78, 299-322.

40. H.A. Witkin, et al., "Stability of cognitive style from childhood to young adulthood," Journal of Personality and Social Psychology, $1967, \underline{7}, 291-300$.

41. Paul Friedberg, with E1len Perry Berkeley, Play and Interplay, New York: Macmillan, 1970, p.16.

42. Samuel Z. Klausner, On Man and his Environment, San Francisco: Jossey-Bass, 1971, p. 64.

43. Heinz Werner, Comparative Psychology of Mental Development, Chicago: Follet, 1948.

44. Robert $\mathrm{K}$. Merton, "The social psychology of housing," in $\mathrm{W}$. Dennis, ed., Current Trends in Social Psychology, Pittsburgh: University of Pittsburgh Press, 1948, p. 181.

45. Aristide H. Esser, "A biosocial perspective on crowding," in J.F. Wohlwill \& D.H. Carson, eds., Environment and the Social Sciences, Washington, D.C.: American Psychological Association, 1972; Parr, 1964, op. cit.

46. Parr, 1966, op. cit.

47. John P. Dean, "Housing design and family values," Land Economics, $1953,29,128-141$.

48. Roger G. Barker \& Herbert F. Wright, Midwest and Its Children, Hamden, Conn.: Archon, 1955. 
49. William F. LeCompte, "Behavior-settings as data-generating units for the environmental planner and architect," in J. Lang, et al., eds., Designing for Human Behavior: Architecture and the Behaviora1 Sciences, Stroudsburg, Pa.: Dowden, Hutchinson, \& Ross, Inc., 1974, p.184.

50. Terrence Lee, "Urban neighborhoods as a socio-spatial schema,". Human Relations, 1968, 21, 241-267.

51. Chalsa Loo, "Important issues in researching the effects of crowding on humans," Representative Research in Social Psychology, 1973, 4, 219-226.

52. Mumford, op. cit.; Meier, op. cit.

53. Harvey Cox, The Secular City, New York: Macmillan, 1966.

54. Lyn H. Lofland, A World of Strangers, New York: Basic Books, 1973.

55. Milgram, 으. cit.

56. Ibid., p. 1461 . 
CHAPTER IV

METHODS

The mathematician, Gauss, wrote a formula in the margin of one of his books, and the note:

"Cuius rei demonstrationem mirabilem sane detexi;

hanc marginis exiquitas non carpert!"

( $I$ have found a marvelous proof for this; but this margin is not big enough to hold it!)

Mathematicians have been trying to prove his formula ever since. 1

\section{CHOICE OF DESIGN}

At the heart of this study is the question, "What adaptations do children make to the density of their home environment?" It has already been seen that there is little reliable data dealing with this specific question, and there is only a relatively small body of literature which deals with it in any fashion. As a result, this study was designed to determine which variables might prove useful in exploring this question, and to determine the range of those variables. This was done through interviews, a constructed test, and primarily through field research using a relatively small group of children.

In the usual paradigm of scientific design, a carefully selected (generally large in number) random sample of a population is subjected to a carefully controlled experimental situation; the resultant behavior is reported in a quantified fashion, and appropriate tests of statistical significance are applied; and the results of these tests are unambiguously interpreted as denying, or failing to deny, a specific hypothesis. Such a design is not the paradigm for this study. Neither the relevant 
variables nor their parameters are clearly defined; the use of the most powerful and the most sensitive of scientific tools is premature. Even though the usual paradigm of scientific design is not used in this study, it should be borne in mind that the choice of one design does not necessarily deny the merits of any other design, for as Kaplan said:

What is objectionable is not that some techniques are pushed to the utmost, but that others, in consequence, are denied in the name of science.... It sometimes even happens that a conspicuously successful technique in some area of behavioral science is not only identified with 'scientific method,' but comes to be so mechanically applied that it undermines the very spirit of scientific inquiry. ${ }^{2}$

Another paradigm in social science is the field-research paradigm In which "parameters and properties are conceptual discoveries, and then, only for theoretical and practical working purposes, are they assigned boundaries." 3 This same paradigm is used by Roger Barker:

The aim of the research must be to discover units that will help to bring order to the innumerable data the behavior stream provides. This has to be accomplished on the frontier of knowledge where guidance by pre-established facts and hypotheses is necessarily minimal, and where investigation must follow the canons of discovery rather than those of scientific verification. 4

The paradigm is defined by Junker as "the task of observing and recording and reporting the behavior of living people in contemporary situations... (with) every intention of avoiding disturbance to their natural activities."5 Field research, then, is a research technique which is designed to identify, to organize, and to set the range of variables in a phenomenal field. It is, in Piaget's colorful phrase, "an effort to see," (une experience pour voir). Its hallmarks are observation which is as unobtrusive as possible, recording which is as detailed as possible, and reflection on the recorded observations (protocols) in the attempt to discover common themes among the protocols. It is best suited for the 
first explorations into new areas of inquiry, where the dimensions, and even the universe of discourse which might contain the dimensions, is unknown. Its results are generally expressed qualitatively, in terms of concepts and variables.

Neither of these paradigms is, by itself, suited to this study. There has been some theoretical work on the central question, and the useful concepts and even some variables have been identified. On the other hand, these variables have not been refined to the point that sensitive statistical tests would not be crippled by large measurement error due to the lack of precisely focused measures. It is apparent that some form of synthesis of these two paradigms must be used. As Kaplan said,

What a scientist does in a particular case may be more or less reasonable, sensible, intelligent. What makes it such is not something in his psychology or ours who are appraising what he does, but something in his problem and in the appropriateness to it of the operations of his understanding. 6

This effort at synthesis is encouraged by Glaser and Strauss' belief that:

...there is no fundamental clash between the purposes and capacities of qualitative and quantitative methods or data. What clash there is concerns the primacy of emphasis on verification or generation of theory.... We believe that each form of data is useful for both verification and generation of theory, whatever the primacy of emphasis. 7

This study, then, draws its methods from the intersection of qualitative and quantitative techniques. Once interesting dimensions have been identified for study, quantification serves to discipline the observation of those dimensions. In attributing discrete values to phenomena, a more useful observation is encouraged, at least along the dimensions being measured. At the same time, qualitative techniques serve to keep in focus which dimensions are relavent. As Schatzman and Strauss put it: The researcher must get close to the people whom he studies; 
he understands that their actions are best comprehended when observed on the spot--in the natural, ongoing environment where they live and work. If man creates at least some of the conditions for his own actions, then it can be presumed that he acts in his own world, at the very place and time that he is. The researcher himself must be at the location, not only to watch but also to listen to the symbolic sounds that characterize this world. A dialogue with persons in their natural situtation will reveal the nuances of meaning from which their perspective and definitions are continually forged. 8

Three procedures were used in this study. The children were observed in their everyday environment, both at a day-care center and at home. Although field observation is generally considered a qualitative method, Barker and Wright's technique for quantifying behavior episodes were also used.9 Second, both the children and their parents were interviewed. The interviews elicited both quantifiable and qualitative information. Third, a test constructed by J.A. Desor ${ }^{10}$ was administered to both a parent and the child. While this test was designed to produce quantified results, an inquiry was performed afterwards to try to elicit the meaning-structures of the test situation for the children. Each of these procedures will be discussed in detail later in this chapter. The point to be made here is that each procedure produced both quantified results and qualitative information about those results. The qualitative Information was used to evaluate the quantitative measures; the qualitative data answered the question, "How well do the measures measure what I want?" The quantitative measures, in turn, provided a base from which to examine the interrelationship of the variables this study is concerned with. In other words, both qualitative and quantitative techniques are integral parts of the study.

Another way to locate the methodology of this study within the sctentific tradition is to place it within Wohiwill's "Programmatic Approach 
to Research on Developmental Change."11 While his schema is designed specifically for the study of human development, it is easily made into a general schema with only minor modifications. Wohlwill distinguished five stages in his schema, stages which are ordered but also all interlinked. His five stages are: (1) discovery and synthesis of dimensions; (2) descriptive study; (3) correlational study; (4) study of determinants; (5) study of individual differences. 12 The general movement is from Stage One to Stage Five, although Wohlwill specifically discusses the possibility of dropping back from a higher stage to a lower stage as new concepts and insights arise and must be developed. This study falls within the second and third stages of Wohlwill's schema. The dimensions of the study (Stage One) were suggested by the existing literature. This study is concerned with describing the range and characteristics of those dimensions (State Two) and their relationships with each other (Stage Three). This study, then, is located well within the tradition of the social sciences, albeit more self-consciously than many studies; for the limitations of this study have been clearly admitted, at the same time acknowledging that the limitations arise from the lack of secure, scientific understanding of the central question. The underlying, basic limitations of the only methodology which is appropriate at this stage of understanding cannot be stressed strongly enough: qualitative and field-observational studies cannot establish the 'truth' of an hypothesis, at least not with one study. As Wohlwill pointed out, "There are, in principle, an indeterminate number of ways of interpreting any difference between subject groups in a nonexperimental study..."13 And Barker pointed out:

The only evidence that reported behavior units actually exist, and are, in fact, self-generated, inherent divisions of the be- 
havior stream, and not products of the methods used by the investigator can be obtained by replicating the observations and analyses. It is only when enough knowledge of the structure and dynamics of the behavior stream has been achieved by patient search, and research, that it may be possible to invoke known properties of the behavior continuum to 'prove' some observation of its characteristics.14

\section{SUBJECTS}

The subjects for this study were $324-$ and 5-year-old children, who were enrolled in the Helen Gordon Child Developement Center. The Center primarily serves the children of students, faculty, and staff at Portland State University, although children of former students can be continued at the Center. Sixteen of the children lived in single-family structures; 16 lived in multi-family structures. The families, while not necessarily established middle-class, are likely to share a middle- and upper-middle class ethos. Seven of the children lived in student-housing apartments; the rest lived in varying qualities of single-family and multi-family housing, depending on the financial means of the family. Because of the training they have received in the day-care situation, the children are likely to be advanced over their peers, especially in the area of peergroup interaction.

Obtaining a pool of subjects for this study posed a problem from the outset. Many of Portland's apartments are located in the Northwest area; but census data shows that few children under the age of ten live there.15 Many of the children under ten live in the Southeast and Northeast areas, but there are few apartments there. Using block data from the 1970 Census, 16 all the blocks in the city of Portland were screened to locate those in which at least 20 percent of its population were under ten years of age and which also had at least one structure with more than ten units. 
The blocks were scattered all over the city, with none concentrated together. To attempt to obtain entry and then acceptance in so many scattered sites which had no common focus could have proven an Herculean task; other options were explored.

The Housing Authority of Portland (HAP) was considered. Although HAP leases units in large apartment structures, it prefers not to lease those units to families with young children; with one exception, the largest struture leased to families with children had ten units. Further, the possible identification by the community of HAP apartments as "welfare housing" was considered to be a dangerous confounding variable. The Portland Public Schools were also considered, but gaining entry proved difficult. The Parochial School System of the Portland Diocese was considered, but it proved to have too few children enrolled who lived in apartments.

Perhaps it is because of the mobility of the student lifestyle, perhaps it is because of the limited available income on many students, or perhaps it is because students have more cosmopolitan (and so, less anti-urban) attitudes; for whatever reasons, about half of the children in the Helen Gordon Child Development Center live in multi-family dwel1ings. The ease of access to a relatively large number of children from multi-family dwellings and the relative homogeneity of their families led to the selection of the older half of the children enrolled at the Center For the pool of subjects.

There were two important limitations imposed on the study by this choice and which weighed against the benefits from the use of these children. The first limitation is that the 'group' cannot be construed as a sample of the residents of Portland, or even as a sample of those resi- 
dents who are college students. The Helen Gordon Center provided a pool of Subjects, and an opportunity to see if predicted effects occur within this group; the findings need not necessarily generalize to any larger population. On the other hand, for what must be considered as the early' stages of a long-term program of research, no more feasible option was available. This limitation was not considered to outweigh its benefits. The second limitation is that the children were all enrolled in a preschool, day-long program: they all have signigicant and extended experience outside the home environment. This means that the failure to find a relationship in this group might not be true of apartment children in general: perhaps a real relationship is effaced by the day-care experience. However, if the relationship is such that six hours a day, five days a week, of group experience can eradicate it, we can expect that the effect of apartment living would not be found in children over 6 years of age (the age for compulsory school attendance) anyway. Further, it is not known how widespread the attendance at day-care is among apartment children. The provision for day-care for all apartment children has already been called for, 17 and planned into many European complexes,18 and could be considered as an ideal, albeit not commonly realized, situation. So, while the limitations to generalization are acknowledged, they are not considered to outweigh the benefits of choosing the children at the Helen Gordon Child Development Center as the subjects for this study. The pool of children consisted of 41 children in the four classrooms of older children at the Center. Of these 41, two were absent for more than a week during the recording of protocols, and had to be excluded from the poo1. Three were excluded when the parents expressed a desire not to be interviewed, and two were excluded when the parents could 
not be reached or a convenient time could not be found. Finally, tivo children were excluded because they had moved recently (within one month), with the result that reliable measures of 'normal' home-behavior could not be obtained. The remaining children (32) made up the group of Subjects for this study.

\section{PROCEDURES FOR COLLECTING DATA}

\section{Obtaining Subjects}

In field study, an important concern is how to obtain access to the subjects, for the conditions under which entry is granted will influence the definition of the situation for both the subjects and for the observer.

In this case, entry was first obtained by meeting with Director of the Helen Gordon Child Development Center. The proposed study was explained both in terms of its concepts and in terms of its demands on the Center and its staff. A written summary of the study was also given to her. She, in turn, explained that the Center has been used for research before, and she had no objections to the study; she also explained that the investigator would need the approval of the individual teachers; and that, although the children were not unfamiliar with being observed (students do observation for class projects, from time to time), the investigator would also need the children's acceptance of his presence. It was agreed, then, that the study would be presented at the next staff meeting; in the meantime, a letter would be sent out to the parents explaining the presence of the investigator (Appendix A). The letter to the parents was sent through the Center--a copy was attached to each child's sign-out sheet so the parent got it when he or she picked up the child at the end of the day. After a length of time to permit any parents to voice objec- 
tions (one and a half weeks), the investigator began to visit the classrooms.

Initially, the classroom visits served to acquaint the children with the investigator, and investigator with both the children and their environment. The investigator would enter the room, sit at the periphery and watch. He would briefly answer questions put directly to him, but asked none himself (while in the room), nor did he initiate any activity. The children were frankly told that the investigator was looking at what they do when they play, and that was why he was there in the room, and that he wanted to watch rather than play. After an hour, he would leave. The schedule of observation was rotated through the free-time periods to get as adequate an impression of the children's day as was possible.

After the classroon observation was well underway, the parents were sent another letter, explaining that an interview was being requested (Appendix B) and that the parents would be contacted by phone or through the mail. A time convenient for the parents was arranged, and the investigator began the interview as soon as the schoolroom observation was completed. At the end of the Interview, he explained the purposes of the study, and answered any questions the parents had. He promised to present his findings orally to the parents as a group, and to send a written summary of the findings when the study was completed. He then requested permission to return and observe the child at home while playing, at a time convenient for the parents.

In summary, the permission of the Director of the Center provided a legitimate position from which to ask the participants for their cooperation. It made negotiation with the individual parents and children much easier, in most cases almost a merè formality. But it also identified 
the investigator with the Center; he was in a position of authority for the children, and was "from the school" for the parents. Once entry had been achieved, the investigator made an effort to distinguish himself from the Center: to the parents, he presented himself as "Erom Urban Studies"; to the children, he consistently refused to grant permissions, referring them instead to their teachers.

\section{Field Observation of Behavior Episodes}

One source of data for this study was drawn from protocols of field observation of the children at play. The object of this procedure was to determine what sort of behaviors the children can be observed to engage in; this need not necessarily be the same as what a child's caretakers (parents, teachers, aides) say he engages in, nor need it even be the same as what the child himself says he engages in. The processes of selective attention, differential reinforcement, and decay of memory traces suggest that even under ideal conditions these three operations would consistently produce some differences; and to these inherent operational differences must be added differences due to the demand characteristics which arise in gathering the data. In the case of field observation, the ideal is unobtrusive, nonparticipant observation. The investigator's height and size made it unlikely that such an attempt would succeed. Instead, a three-week period of initial contact at the day-care center served to acquaint the children with him, in the hope of making later observation less obtrusive. During this period of initial contact, no quantitative data were recorded, although impressions and qualitative descriptions were noted, after leaving the setting.

Field observation was carried out in two different setitings: the 
Helen Gordon Child Developement Center, and the child's home. At the day-care center, the children were observed during periods of free play (in the morning, before scheduled activities began; and in the early afternoon, a scheduled period for free play) in their own classrooms or * in either of two activity rooms (the craft shop and the block room). The children were not observed in the outdoor playground because the use of the playground was restricted to only a portion of the time in which the children were observed. It was feared that this restriction would contaminate the data, which was intended to be gathered in a setting to which all children had equal access. At the child's home, each child was observed during what was reported by the parents to be a common time for the child to be free to play as he wished. Accordingly, the child was observed wherever he happened to be in the home setting at that time--inside or outside, alone or with friends.

The purpose of observing the children in the home was to gather data on how the child typically spends his free time at home; since differences in the setting are the independent variables in the study, it made no sense to enforce some sort of commonality of setting across children. An effort was made to hold other environmental factors constant: no child was observed when the weather made outside play impossible; when multiple times were available for observation, apartment and single-family children were matched on the time dimension.

The purpose of observing the children in the day-care setting was to obtain information on their play in a common setting. Such data would permit an estimate of the generalization of typical behavior in the home setting to other settings. Another way of phrasing the same concern is: the data could provide an estimate of a child's behavior in a standard 
environment: changes in behavior between the two settings would be due to environmental pressure while continuities in behavior are more likely to be due to personality organization. Another advantage to field observation in the day-care setting was that it provided an opportunity to observe the children, and develop a schema for each child's typical behavior, without first knowing whether the child lived in an apartment or a single-family home. Such a procedure weakens a chronic complaint against field studies, that the effect observed may be due to the experimenter's expectations.

Protocols of behavior episodes were recorded in each of the two field settings, at the day-care center and at the home. In the case of the day-care center, only one protocol per child was recorded on any given day until a total of ten protocols were collected. In the event that a child was sick for several days, two protocols might be recorded on the same day, but they were always separated by at least five minutes of unrecorded behavior. In the case of the home, five protocols were recorded on two separate days, once inside and once outside, if possible. Each protocol was separated by at least five of unrecorded behavior. The children were observed on only two separate occasions in order to minimize the inconvenience to their parents, and to maximize the investigator's travel time, since in only a few cases did the children live near enough to play with each other. Two occasions were used rather than one. in order to avoid having an entire record distorted by an unusually good or an unusually poor day for any child.

The procedure for recording the protocols was as follows. The investigator entered the setting and chose a position on the periphery of the setting which permitted observation of the entire setting. He carried 
a clipboard and a stopwatch. The investigator first recorded the names of everyone present in the setting. If several children were present (i.e., at the day-care center), the investigator observed each child in a predetermined, random order which was changed for each occasion. A protocol was begun when the child clearly entered a new episode; usually, this involved a change of location or change of materials played with, or change in the direction of behavior. The actions were recorded and their time noted in seconds. The protocol continued until the episode had been clearly and unambiguously ended; usually, this involved a change of location or change of materials played with, or a change in the direction of behavior. Whenever the field situation was ambigous, more rather than less data was recorded. When observing at the home setting, the investigator waited five minutes and then observed the child again. When a new behavior episode was clearly begun, a new protocol was recorded. When observing at the day-care setting, the investigator selected the next child on the list, waited until a new behavior episode was clearly begun, and recorded the protocol. If the child was engaged in a behavior episode that was already ongoing, and the child continued with that episode for at least two minutes and showed no signs of completing the episode shortly, the investigator would select the next child on the list and repeat the procedure. At the end of each day, the protocols were typed up for later coding.

\section{Interviews}

A second major source of data was an Interview in the home with both a parent and the child. The interview was made at the home, rather than at the day-care center, for two reasons: the questions all pertained 
to the home environment, and it was expected that the environment itself would serve as a memory-aid in answering the questions; second, it served to introduce the investigator into the child's home, hopefully accustomIng the child to the investigator's presence there, and minimizing disruption of routine when the investigator returned later to observe the child at play. The interview was arranged with whichever parent was listed at the day-care center as responsible for picking the child up at the end of the day (usually, the student).

The formal interview (Appendix $C$ ) consisted of three sections. The first section obtained factual information about the home environment: the family, the building, and the neighborhood. These questions were all concerned with matters of fact. The only questions that were answered with any hesitation were the size of the unit and the number of children In the neighborhood. Usually, the parent was able to make an estimate of gross floor area that concurred with the investigator's impressions. In the case of the number of children, not infrequently the parent would close his eyes and count the children, visualizing the units in the neighborhood. The only exception was the case of a large student-housing apartment building. In that case, the Information was taken from Portland Student Services, the managers of the building. The second section obtained the parent's opinions of his child's play. Most parents had difficulty estimating how much time the child spent in solitary play. The first response was usually, "He plays alone a lot," or "He almost never plays alone." A more exact estimate was difficult, and frequently represented an arbitrary number--"about a third," "very little, maybe 5 percent." The other two questions, how many children the child plays with and where, were easy to answer. The third section obtained the child's 
perception of how many children he played with and where. At the end of the second section, Desor's test was administer ad to the parent; it was administered to the child at the end of the third section. In order to avoid contaminating the child's responses with the parent's responses, ' the child was given crayons and paper and asked to draw a picture; while the child was drawing, the parent was interviewed.

Following the formal interview, the investigator explained the purpose of the study and answered any questions the parent might have. This also provided the opportunity for an informal, unstructured interview. The parent was asked for his impressions of the child's activity at home, for his opinion of the type of living situation he was in and its effect on the child. Any remarks made were recorded after leaving the home, in order to avoid formalizing the situation.

\section{Desor's Test}

A third source of data was the child's performance on a test for perception of crowding designed by J.A. Desor. 24 The test was originally designed to determine how variations in the architectural design of a room (including area) would influence an adult's perception of the room as being 'crowded.' The test consisted of a box, open at the top, and a number of clothespin-figures. The dimensions of the box were varied, the location of doors and partitions varied. The subject was requested in each instance to put in as many figures as he could, without making the room crowded.

Desor's test for perception of crowding was modified for the purposes of this study. In the first place, this study is focusing on group differences in the perception of crowding, rather than attempting to dis- 
cover architectural correlates of crowding. Accordingly, only one standard stimulus was presented, Desor's "medium-sized" room. Second, adjustments had to be made for the developmental stage of the Subjects. Piaget has shown that young children are not able to shift visual perspectives and still maintain the same schema. 25 Piaget's study suggests that a child, looking from the top into a model of a room, might not be able to translate that perception into his experience of a room, which is generally seen from the side. Support for this suspicion can be found in the common design for a doll's house: one wall is cut away, providing a more nearly identifiable visual perspective for the child. Accordingly, the box, or model of a room, was designed to have both the ceiling and one wall cut away. Also, the figures were scaled to represent a child's height, roughly 45 inches tall. The concern for the developmental stage of the child also resulted in a modification of the instructions. Desor suggested that a common situation be specified, one that can be generalized from children to adults; and she suggested that the instructions be simplified to avoid misunderstanding of the word 'crowding.'26 The room, then, was specified as "a room where children play together"; and the child was asked to "put as many children in the room as you can, without there being too many."

The apparatus consisted of a box, $6 \times 12$ inches and 6 inches high, with a floor, two short sides and one long side. The other side and the top were absent. A door was drawn on the middle of the 1ong wall. The box was designed to collapse flat so it could be easily transported. In a second box were 46 figures, 2 1/2 inches tall, made from 1-inch dowel cut into $13 / 4$ inch lengths and a wooden bead glued on top, with painted faces and arms but no indicators of sex or any other role. Forty-six 
figures were supplied so that all the available figures could not be placed in the room without obviously overloading the room; shoulder-toshoulder, the figures filled almost three quarters of the room.

The apparatus was then pretested, using eight pre-school children, age $33 / 4$ to 6 . The 3 year old could not understand what was requested, no matter how the request was reworded. The 5- and 6-year-olds had no trouble understanding the request at all. The 4-year-olds had some difficulty with the test, two succeeding and one failing to understand the task. Several modifications in the procedure resulted from the pre-test. First, no child who put all 46 figures in the box was able to repeat the instructions. Invariably, they were trying to put all the figures in the box, without regard for crowding the figures. Second, some children who put all the figures in the box, when asked if it was crowded replied, "Yes"; and when asked to take out "just enough children to make it not crowded," proceeded to do so, leaving in less than 30 figures. Thirdly, the instruction "without there being too many," was ambiguous to the children. Two of the eight (one was a 4-year-o1d) spontaneously said, "You mean so it's not crowded?" Even the 3-year-old understood the word "crowded," and could identify a 'crowded room,' even though she couldn't simultaneously "put in as many as (she could)." As a result, the instructions were changed to "Pretend that this is a room where children play together, and that these are children. Put as many children in the room as you can, but don't put in so many that they get in each other's way. Don't make the room crowded." Further, once the child indicated he had finished, he was asked if the room looked crowded. If he replied that it was, he was asked, "Take some out until it isn't crowded any more." If the child still replied that the room was not crowded even though 46 fig- 
ures were in it, no further questions were asked and the response was coded as a failure to understand the directions.

Desor's test was administered first to the parent, at the end of the formal interview with the parent. It was administered to the child at the end of the formal interview with the child. To avoid contaminating the child's response to the test with his parent's, the child was given crayons and paper and asked to draw a picture while the investigator talked to the parents. If the child refused to draw, or if the child finished drawing before the test was administered to the parent, the test was described to the parent and permission was asked to administer the test to the child first.

\section{OPERATIONAL DEFINITIONS}

The terms used in stating the hypotheses will now be defined in terms of the operations used to measure them. The terms are divided into two groups, those relating to the housing environment (independent variables) and those relating to the child's behavior (dependent variables). The housing environment is further categorized in terms of its physical, socio-spatial, and social dimensions. The child's behavior is categorized as withdrawal from interaction, filtering potential interaction, and cognitive structuring.

\section{Physical Dimension of the Housing Environment}

Dwelling-Type. Single-family or multi-family dwellings, determined by the number of individual units in a free-standing structure. It is measured by the number of mailboxes at the entryway of the building. It wiIl be abbreviated as ( $\ddot{F}$ ÜNITS). 
There are other characteristics of multi-family dwellings that are not used for testing the hypotheses, but are used in descriptive sections of the study:

1) Type of external access: Presence or absence of inside stairway or hall to individual units in a multi-family dwelling, It will be abbreviated as (STAIRS).

2) Number of units per hallway: When access to the outside is by means of a stairway or hall, the number of units on the stairway or hall is counted. It will be abbreviated as (\# UNITS/HALL).

3) Number of floors in structure: Includes basement, if housing units are leased there. It will be abbreviated as (非LOORS). Number of rooms in unit. Total number of rooms in dwelling, excluding bathrooms, halls, and unfinished attics or basements, or porches. It will be abbreviated as (\# ROOMS/UNIT).

Size of unit. Estimate of gross floor area, in square feet. It will be abbreviated as (SIZE OF UNIT).

Socio-Spatial Dimension of Housing Environment

Contact-facilitating characteristics.

1) Total number of people in the structure, as estimated from interview of parents. It will be abbreviated as (非 PEOPLE--BLDG.).

2) Total number of people in the child's home range, as determined by the 1970 Census of block statistics. 27 It will be abbreviated as (\# PEOPLE--NBRHD). The child's home range was determined from the interview with the parent.

Interaction-potential characteristics.

1) Number of peers in the structure, as estimated from interview of 
parents. It will be abbreviated as (非 PEERS--BLDG).

2) Number of peers in the child's home range, as estimated from interview of parents. It will be abbreviated as (非 PEERS--NBRHD).

\section{Social Dimension of Housing Environment}

Number of siblings. Children living in same housing unit as the child, as determined from interview of parents. It will be abbreviated as (\#SIBS) .

Number of adults. Adults responsible for daily care of child, as determined from interview of parents. It will be abbreviated as (\#ADULT).

Social class of housing environment. Measured by social-class ranking (in quintiles) of census tract in which dwelling is located. It will be abbreviated as (NBRHD SES). Source of measure: Population Research Center. 28

Social class of origin. Measured by occupation of child's parents, or child's grandparents if parents are students. It will be abbreviated as (SES). Source of measure: McGuire-White Scale (Appendix D).

Financial ranking of family. Measured by source of income for the household. It will be abbreviated as (FINANCE). Source of measure: McGuire-White Scale (Appendix D).

Withdrawal From Interaction

Rejection of overtures for interaction.

1) Frequency of ignoring verbal or unambiguous kinesic initiations of interaction, as determined by field observation. It will be abbreviated as (IGNORE).

2) Frequency of refusing verbal initiations of interaction, as determined by field observation. It will be abbreviated as (REFUSE). 
Solitary play. Any activity, whether alone or in the presence of others, which is carried on without verbal or other behavioral interaction with others. In other words, any activity in which the presence of other is a matter of indifference.

1) Percent of time spent in solitary play, as determined by field observation. It will be abbreviated as (\% SOLITARY).

2) Reports of percent of time in housing environment spent in solitary play, as determined by interview of parent. It will be abbreviated as (P-SOLITARY).

\section{Filtering Potential Interaction}

Time allocated to behavior episodes. Duration in seconds of any activity pattern which manifests constancy of direction, normal behavior perspective, and superior whole potency. It will be abbreviated as (DURATION). For an explanation of the criteria of 'behavior episode,' see the section on "Coding Protocols" (p. 93).

Selection of potential playmates.

1) Ratio of the number of peers in the play-group to the number of peers present in the setting as measured by field observation. It will be abbreviated as (\% PEERS) .

2) Number of peers in the play-group, as determined by field observation. It will be abbreviated as (\# PEERS).

3) Number of peers in play-group, as determined by interview of parent and of the child. They will be abbreviated as (P-PEERS) and (CPEERS), respectively.

Use of Settings. A setting is defined as a physical milieu in which an action occurs and which shapes and supports that action. Any 
action occurs in some physical milieu and must be adapted to the milieu. Since a behavior episode may be composed of several distinct actions, it also may occur in several distinct settings. Since the setting is reciprocally tied to the action, and since space is indefinitely divisible, any given space is comprised of an indefinite number of settings.

1) Number of settings used in play, as determined by field observation. It will be abbreviated as (SETTINGS).

2) Number of settings listed by the child in response to the question, "Where do you play?" It will be abbreviated as (PLACES).

3) Area in square feet of the space described by the parent in response to the question, "Where does your child usually play?" It will be abbreviated as (RANGE).

Cognitive Structuring

Tolerance for social density. Performance by the child on Desor's test. Results are scored as the number of figures placed in the apparatus. It will be abbreviated as (DESOR).

\section{PROCEDURES FOR ANALYZING DATA}

\section{Coding Protocols}

In any observational research of natural settings, an important concern must be to establish the public nature of the data obtained. As in astronomy, a physical science which relies on naturalistic observation, the test of such data is in duplication. A frequent method in astronomy is for two independent observers to observe the same phenomenon from different locations. The results are corrected for difference in location and then compared. Such a procedure was not practical in this study. It 
was feared that two strange adults could not be absorbed by a classroom of only ten children and two teachers: the presence of one investigator was disruption enough. In gathering behavioral data in natural settings, the more common method for establishing the public nature of the data is replication: rather than duplicating the observations at the same time, they are duplicated in series. In fact, Roger Barker claimed that this is the principal method. 29

Since all perception is a process of selection, and since an ambiguous action might be inaccurately labeled in a protocol, the confirmation of observational accuracy is not a trivial question. But it is not a question which is confined to naturalistic observation of human behavior: a meter can be misread, the time at which a star passes a point in the heavens can be poorly estimated, ambiguous behavior in the psychology laboratory can be misinterpreted. The fallibility of human observation (and human observation must occur at some point in any data-gathering) is inherent in any human understanding.

Confirmation of the accuracy of the observations reported in this study must await replication in another study. However, the accuracy of the interpretation of the protocols of the observation can be confirmed in this study, if two investigators simultaneously and independently code the protocols. The coding can then be compared. This requires a set of guidelines by which the protocols must be coded, and a technique for evaluating the agreement of the two investigators.

A formula for estimating the accuracy of the coding of behavior episodes has been provided by Barker and Wright: 30 


$$
\begin{array}{ll}
\text { Estimate of }= & \begin{array}{l}
\text { Number of Episodes Discriminated by X } \\
\text { Which Were Also Discriminated by } Y
\end{array} \\
\text { Accuracy } & \text { Total Number of } X \text { Total Number of } \\
\text { Episodes Dis. by } X+\text { Episodes Dis. by } Y &
\end{array}
$$

This can be more simply stated:

\begin{tabular}{ll} 
Estimate of & $\begin{array}{l}\text { Number of Episodes } \\
\text { Agreed on }\end{array}$ \\
\cline { 2 - 2 } Accuracy & Mean Number of \\
& Episodes Coded
\end{tabular}

In this study, rather than an entire behavior-stream, individual behavior episodes were selected for coding. This means that the "Mean Number of Episodes Coded" was a predetermined number; the meaning of "Number of Episodes Agreed on" comes to be "Number of Episodes Which Included All the Same Actions."

The guidelines for evaluating the protocols were derived from Barker and Wright's Midwest and Its Children. 31 According to them, a behavior episode is determined by three criteria: constancy of direction, occurrence within the normal behavior perspective, and superior whole potency.

Constancy of direction means that all the action in a behavior episode runs toward or is aimed at the same goal. Barker and Wright suggested that the goal is a particular, terminal position, and that the process of episoding has two parts: diagnosing the end toward which the person is moving, and judging whether the 'movement' brings the person nearer to that end. Barker and Wright's discussion of behavior episodes was in the context of dealing with day-long protocols, in which the time was measured in minutes. A pretest of the coding guidelines, which used briefer, five-minute protocols in which time was measured in seconds, indicated 
that not all the behavior of 4 - and 5-year-old children envisioned a clearly intended terminal position. Segments of behavior, some as long as five minutes, could only be described as "standing around," "walking around," or "looking for something to do." This behavior is known to most mothers in the form of the child's question, "How come there's nothing to do?" As a result of the pre-test, undirected activity was coded as a behavior episode if it persisted for more than thirty seconds and its end-point was considered to be the discovery of some specific goaldirected activity that persisted for at least thirty seconds. The thirtysecond criterion was imposed because children were frequently observed to mill around briefly before settling down to a specific activity that had apparently been intended all along; it was also imposed to ensure that what appeared to be non-directed activity was not goal-directed activity for which the goal had not yet become apparent. In summary, then, constancy of direction means that all the action in a behavior episode runs in the same direction, either toward a goal or in search for a goal.

Barker and Wright list seven clues for the recognition of the direction of a behavior episode. 32 They are: 1) action which persists; 2) action is renewed after a forced digression or delay; 3) actions which are preparatory adjustments to the goal accompanying the action; 4) sustained locomotion is abruptly discontinued when the goal is reached; 5) action between beginning and end points shows continuity; 6) action from beginning to end follows the shortest available path; 7) variance in behavior parallels variance in an environmental objest. These seven clues served as the basis for determining the length of a behavior episode. As long as one of them was present, the action was coded as continuing in a constant direction. In the field observation, however, these 
seven clues were too many to help to decide whether to begin and whether to terminate a protocol. Harold Dickman, studying people who are untrained in the use of behavior episodes, found that his subjects used three cues to determine when a train of behavior had been completed. 33 . They are: 1) change in physical direction of behavior; 2) change in behavior cbject 'commerced with'; 3) change in behavior setting. Dickman's three clues were used as a guide for the field observation, with the intention that a more refined analysis would be done on the protocols later. The second guideline is that the action should occur within the normal behavior perspective. It is concerned with the size of the behaviorepisode unit; the smallest unit of the stream of behavior must be able to be immediately recognized as a unity. It is defined as "a molar behavior unit which the person himself perceives to be in progress." 34 In other words, it is what the person would tellyou he is doing if you were to ask him. Barker and Wright point out that, like constancy of direction, a "normal perspective" is defined in terms of what exists for the subject; it cannot be directly observed. At bottom, this is a problem for all classification: to the unitiated, the purpose of an automobile brakedrum puller is not apparent; for that matter, it is not always apparent that some structures are intended to be chairs rather than purely ornamental sculpture. Usually, the problem of classification is encountered in cross-cultural studies where there is less shared context, less apperceptive mass which can be drawn on to surmise another person's intentions. 35 Barker and Wright clearly admitted this, saying that one's skill in determining the length of an episode is influenced by acquaintance with the subjects, common cultural background with the subjects, and familiarity with the behavior settings and behavior objects which are used. 
Two working guidelines for coding the normal behavior perspective of the protocols can be taken from Barker and Wright. First, change in is behavior perspective generally parallels change in the direction of behavior. Second, if there is no positive evidence that the child is aware of a more remote terminal intended position, the episode should be considered ended. In other words, if a child completes an action and begins another, and there is no indication that he perceives the new action as coordinated with the earlier one, then the episode is ended.

The third guideline is that the episode as a whole must absorb more of the actor's attention than any of its constituent parts: the potency of the whole must be greater than the potency of its parts. If any action or set of actions absorbs more attention than the original goal of the episode, a new, more powerful goal has been established, and thus a new behavior-episode has occurred. A flash of anger, resulting in a verbal or physical fight, for example, would result in superior part potency and thus a new episode would be coded; the introduction of a novel stimulus into the setting (a stranger or a new toy) could also lead to superior part potency. It is also possible, however, that within any given goal one or more subgoals may arise: getting dressed could be broken down into "putting on a shirt," "putting on pants," "putting on socks," and "putting, on shoes." These sub-goals would then constituted "enclosed" episodes within the larger behavior episode. The decison, in other words, must be made as to whether the part-goal is going in the same direction or is advancing the same end as the overall goal.

When the protocols were coded, they were coded until the behavior episode was terminated, either by success, failure, or interruption; that Is, until the episode no longer commanded the actor's attention. This 
does not mean, however, that only one behavior episode will be coded.

Human behavior is rarely unidimensional and unambiguous; and even when a specific action is unambiguous, human beings are adept at doing two or more things at once. As Barker put it,

The units of the stream of behavior are not independent and static like a course of stepping stones; they are interdependent to various degrees.... Enclosing-enclosed structures ...are of crucial importance here...."36

Besides enclosing-enclosed episodes, episodes may also be interlinked, where one episode begins before an earlier one has ended. In coding the protocols, when the episode which began the coding is terminated, coding is terminated. Enclosed episodes are coded as such, and that part of an interlinked episode that coincides with the behavior episode in question is also coded.

Once the length of the behavior episode has been determined by following the above three guidelines, the behavior episode was examined and the values of certain variables were coded. The variables were: the number of settings used in the episode, the number of peers and the nunber of people in the child's play-group in the episode, the number of times the child ignored an offer of interastion, and the number of times the child refused an offer on interaction. These measures have already been defined in the section, "Operational Definitions."

In summary, the procedure for coding the protocols was as follows: Two investigators coded ten protocols together, applying the three guidelines for behavior episodes. Following this, fifty protocols were coded separately, using the three guidelines. Inter-coder reliability was measured by Barker and Wright's formula. Enclosed episodes were not included in the estimate of reliability. The instructions to the inde- 
pendent coder are found in Appendix D.

When a completely naive investigator was used in the coding, a student who was familiar with neither Barker's work nor with the situation in which the observations were made, a reliability coefficient of 0.76 was obtained. The author and the independent coder agreed three-quarters of the time. When a second investigator was used incoding, a classmate who was familiar with Barker's work but not familiar with the situation in which the observations were made, a reliability coefficient of 0.86 was obtained. While agreement was not complete, it was considered adequate, especially in light of the minimal training in the specific techniques used.

Descriptive Analysis

Before turning to an analysis of the hypotheses, it is necessary to evaluate the measures that will be used in testing the hypotheses. The data from the field observation consists of ten observations for each child in each environment. These data are then averaged for each child, with the result that all measures drawn from field observation are the mean score of the ten observations of the child. This serves to lesser the weight of extreme scores without totally eradicating them, and it provides a convenient summary score for each child. Measures of the dependent and independent variables will first be analyzed for validity using a multitrait-multimethod matrix. The independent variables will also be analyzed to determine their usefulness as descriptors of dwellingtype (single-family or multi-family structures).

The multitrait-multimethod matrix described by Campbe11 and Fiske 37 is basically a technique for organizing the familiar correlation matrix 
so that the entries fall together in some meaningful pattern. This pattern may then be used to determine whether similar measures cluster and different measures diverge. The specific procedures for analyzing the matrix are more easily grasped when illustrated both schematically and empirically, and will be explained in the next chapter where a matrix will be available for reference. The main importance of the technique, however, is to establish the convergent and the discriminant validity of measures of the same trait. Convergent validity entails confirmation by independent measurement procedures. Discriminant validity entails distinguishing groups of measures from each other.

Some interesting methodological points are brought out in using the multitrait-multimethod matrix. In the first place, such a procedure involves a looser sense of the meaning of operationalism, for it implies that different operations can measure the same variable. This would further suggest that what is expected in a multitrait-multimethod matrix is convergence, not congruence. The same phenomenon, examined from different viewpoints, should result in similar but not identical results. The full impact of this position is not apparent even in Campbel1 and Fiske's paper, for they stated that:

The presence of method variance is indicated by the difference in level of correlation between parallel values of the monomethod block and the heteromethod blocks, assuming comparable rellabilities among all tests. 38

And again they stated: "(A) 1iterary definition... is now best represented In what (the) independent measures of the trait held distinctively in common."39 In other words, what is common is the true defintition, the rest is error. The concept of convergence, however, and the looser understanding of operationalism suggests that variance not in common may not be 
due to error but due to the (necessary) inability of a single operation to grasp all the dimensions of any phenomenon, although human experience can and does take them all into account.

Two points should be made about the interpretation of a multitraitmultimethod matrix. First, the convergent validity values usually will not be interpretable in any absolute fashion. To do so would require that both the traits and the methods were completely independent; it would mean that the relationship to all measures of other variables would be zero. In most cases, this cannot be expected to occur and one would consider instead the relation of the validity values. Second, the failure to arrive at convergent validity between two variables can be due to any of three situations: 1) neither method is adequate for measuring the variable; 2) one of the two methods does not really measure the variable; 3) the variable is not a functional unity. The second possibility can be evaluated by examining the correlation with measures of other variables, to see if there are high values for one of the methods. If measures of a trait by one method are highly correlated with measures of other traits, it would suggest that the method is really measuring one of the other variables. Failure to find this evidence, however, does not rule out the possibility that the method is really a measure of some other variable which is not included in the matrix. There is also no way that the first or third possibility can be evaluated by examination of a multitrait-multimethod matrix.

The second step in the Descriptive Analysis will be to examine the independent variables in terms of the single-family/multi-family dichotomy. The purpose of this procedure is to describe the dwellings in terms of their group-characteristics and to determine whether these character- 
istics are significantly different between them.

The significance of the differences of the characteristics of the groups will be analyzed using a t-Test for the difference of means. Use of this test assumes two independent random samples: that is, two samples must be drawn independently and in a random fashion from the relevant population. Since the test is concerned with the differences from a mean, the assumption of normality is assured given a large enough sample, by the Central Limit Theorem. Guilford suggests that a sample of 30 is of adequate size. 40 The assumption of independence and randomness are necessary to insure that the test does not produce inaccurate results due to the suppression of variance which is the concomitant of correlated samples. These assumptions are not as stringent as they might first appear, for as Blalock pointed out, as long as a single larger sample is drawn randomly from a population, and then conceptualized in terms of several distinct and independent samples, the assumptions of independence and randomness may be considered fulfilled. 41 These two assumptions are also, satisfied for this study.

The use of any significance test, and especially a parametric test, in this study requires some explanation. The problem is that, strictly speaking, the subjects of this study were not a sample drawn from some Identified larger population. Except for those individuals who refused or who could not be contacted, the subjects of this study are the entire population of older children at the Helen Gordon Child Development Center. Thus, any difference observed is a 'significant' difference in the sense that there was no sampling error (since there was no sample).

There is still a sense, however, in which significance tests are appropriate. As Blalock pointed out, significance tests can be used with 
populations to rule out the possibility that the observed differences are due to "chance-processes" as an alternate explanation. 42 In using the significance tests in this fashion, one assumes an infinite, normal universe of chance-processes, of which the population is a 'sample.' The use of an entire population also weakens the importance of the randomness assumptions, since there can be no 'error' due to biased sampling. This does not, of course, do away with the problem of generalizing the results to some other population. Nor does it rule out the possibility, inherent in any 'natural' (as opposed to 'experimental') study, of spurious results (due to covariance with some third variable) which occur because of selfselection. The problems, however, were fully recognized and have not been considered a threat to this study, which is primarily intended to estab1ish the possibility of a relationship.

The model used in this test is that

$$
t=\frac{\bar{x}_{1}-\bar{x}_{2}}{\hat{\sigma}_{\bar{x}_{1}-\bar{x}_{2}}} \quad \text { where } \quad \widehat{O}_{\bar{x}_{1}-\bar{x}_{2}}=\sqrt{\frac{s_{1}^{2}+s_{2}^{2}}{N_{1}-1}+\frac{N_{2}-2}{N_{2}}}
$$

This model assumes as a null hypothesis that the means are equal, but makes no assumptions about the equality of the standard deviations. Although this model can be less efficient than one which also assumes equal standard deviations, it was not considered justifiable in this case. The two groups are of equal size, and the total number of cases is large enough to avoid the usual small-N problems with this form of the t-Test. Since the two outstanding weaknesses of the model are avoided, there is no reason to accept an additional assumption which might introduce additlonal error into the analysis, especially since in cases where the standard deviations are equal, the models are identical. 
Analysis of Hypotheses

The three hypotheses will be analyzed using Pearson's product-moment correlation, $\underline{r}$. For every coefficient the size of the relation and its significance will be reported. The test for significance is to de- ' termine whether the observed relationship can be explained by chance. The correlation coefficient is a measure of the strength and direction of the observed relationship. Where a measure of relationship is required between one of the dichotomous independent variables and one of the dependent variables (all of which were measured on at least an interval scale), a point-biserial correlation was obtained. 43

Hypothesis $2 b$ predicts an 'inverted-U' function of the regression line. This will be analyzed by employing curve-fitting procedures.for second-order models. 44 A regression model ril1 be tested using the formula $Y=b_{0} t b_{1} X+b_{2} X^{2}+e$

After an hypothesis is tested in the form in which it was stated, it will also be tested in alternate forms, using other measures which were highly correlated to the specified measures. It should be borne in mind that measures of those relationships, tailor-made to the data as they are, are intended to be simply descriptive tools. 
NOTES

1. Quoted from Prof. John C. Sallis, classroom lectures in Symbolic Logic, Spring Term, 1972, Duquesne University.

2. Abraham Kaplan, The Conduct of Inquiry, San Francisco: Chandler, 1964, p. 29.

3. Leonard Schatzman \& Anselm L. Strauss, Field Research: Strategies for a Natural Sociology, Englewood-Cliffs, N.J.: Prentice-Hall, 1973 , p. 2 .

4. Roger G. Barker, "The Stream of Behavior as an Empirical Phenomenon," in R. Barker, ed., The Stream of Behavior, New York: Appleton, Century, Crofts, 1963, p. 10 .

5. Buford Junker, Field Work, Chicago: University of Chicago Press, 1960, p. 2 .

6. Kaplan, 으. cit., p. 18 .

7. Barney G. Glaser \& Anselm L. Strauss, The Discovery of Grounded Theory, Chicago: Aldine, 1967, pp. 17-18.

8. Schatzman \& Strauss, op. cit., pp. 5-6.

9. Roger G. Barker \& Herbert F. Wright, Midwest and Its Children, Hamden, Conn.: Archon, 1955.

10. Jeanette A. Desor, The Psychology of Crowding: An Experimenta1 Investigation, unpublished Ph.D. dissertation, Cornell University, 1969.

11. Joachim Wohlwil1, The Study of Behavioral Development, New York: Academic Press, 1973.

12. Ibid., p. 40 .

13. Ibid., p. 37 .

14. Barker, 으. cit., p. 17 .

15. Center for Population Research and Census, "Population Pyramids by Census Tract for Portland Part of Portland SMSA," Portland State University, mimeo, n.d.

16. U.S. Bureau of Census, Census of Population and Housing: 1970 Block Statistics, Washington, D.C.: U.S. Government Printing Office, 1971.

17. Arvid Bentsson, Environmental Planning for Children's Play, New York: Praeger, 1970, p. 13 . 
18. Paul Chombart de Lauwe, Famille et Habitation, Tome II, Paris: Centre National de la Recherche Scientifique, 1960.

19. Barker \& Wright, 으. cit.

20. Ibid., p. 5 .

21. Horace, Odes, I, 28, 16 (my translation). The Latin text reads, "Omnis una manet nox."

22. Barker \& Wright, o․ cit., p. 6.

23. Ibid.

24. Desor, op. cit.

25. Jean Piaget \& Barbel Inhelder, The Child's Conception of Space, New York: Norton, 1967.

26. Private communication.

27. U.S. Bureau of Census, Census of Population and Housing: 1970 Census Tracts, Washington, D.C.: U.S. Government Printing Office, 1972.

28. Center for Population Research and Census, "1970 Census Tract Data for Clackamas, Multnomah, and Washington Counties," Portland State University, mimeo, n.d.

29. Barker, op. cit., p. 17.

30. Barker \& Wright, op. cit., p. 271.

31. Ibid., pp. 236-270.

32. Ibid., pD. 238-239.

33. Harold Dickman, "The perception of behavioral units," in R.Barker, ed., The Stream of Behavior, New York: Appleton, Century, Crofts, 1963, p. 39 .

34. Barker \& Wright, 으. cit., p. 244.

35. Cf. Michael Cole, et al., The Cultural Context of Learning and Thinking, New York: Basic Books, 1971 .

36. Barker, op. cit., p. 12 .

37. Donald T. Campbe11 \& Donald W. Fiske, "Convergent and discriminant validation by the multitrait-multimethod matrix," Psychological Bulletin, $1959, \underline{56}, 81-105$. 
38. Ibid., p. 85 .

39. Ibid., p. 101.

40. J.P. Guilford, Fundamental Statistics in Psychology and Education, 4th Ed., New York: McGraw-Hil1, 1965.

41. Hubert M. Blalock, Social Statistics, 2nd Ed., New York: McGraw-Hill, 1972, p. 220

42. Ibid., pp. 238-239.

43. As Guilford, op. cit., p. 322, pointed out, a point-biserial $\underline{\mathbf{r}}$ is computed automatically by the computer program for product-moment correlations.

44. N.R. Draper \& H. Smith, Applied Regression Analysis, New York: Wiley, 1966, pp. 129-130. 
CHAPTER V

\section{RESULTS: ANALYSIS OF THE MEASURES}

...in war there is no victory which cannot be regarded as unsuccessful, for the objective which one aims at is the total annihilation of the enemy and this result is never attained; yet there are wars which are won and wars which are lost. So it is with any activity; failure and success are two aspects of reality which at the start are not perceptible. 1

Before the hypotheses are analyzed, the measures which will be used in the analysis must be evaluated. The validity of the measures will be evaluated through multitrait-multimethod matrices. The measures of the independent variables will be further evaluated in terms of their relation to the single-family/multi-family dichotomy.

\section{MULTITRAIT-MULTIMETHOD MATRIX: DEPENDENT VARIABLES}

The multitrait-multimethod matrix has been described by Campbell and Fiske as a technique for examining the validity of measures. Validity must be stablished from two directions: both convergent and discriminant validity must be shown. According to Campbell and Fiske:

Any conceptual formulation of a trait will usually include implicitly the proposition that this trait is a response tendency which can be observed under more than one experimental condition and that this trait can be meaningfully differentiated from other traits. 2

Convergent validity then entails confirmation of a trait by independent measurement procedures; discriminant validity entails distinguishing groups of measures from each other. In order to evaluate these concerns, 
a matrix must be developed in which 1) a measure or set of measures is treated as a trait-method unit and 2) more than one trait and more than one method must be employed. In this study, three methods were used to obatin most of the measures: interviews, observation in the home environment (abbreviated, HOME), and observation in the day-care environment (abbreviated SCHOOL). To be able to evaluate the possibility that only the behavior inside the home would be comparable to behavior inside the day-care center, a subset of the home observations were examined separately. This subset was all the observations made inside the home, and is abbreviated (INSIDE). Using these three methods, two variables were measured: withdrawal from interaction, and filtering potential interaction. Each variable is composed of traits: withdrawal is composed of rejecting interaction and solitary play; filtering is composed of duration of play, size of play-group, and number of settings used. In mañzy cases, more than one measure of each trait-method unit is employed (e.g., number of peers in play-group and proportion of peers in play-group). In addition to these multitrait-multimethod units, a third variable, cognitive structuring, composed of only one trait, was measured by only one method, the test for tolerance for social density.

A multitrait-multimethod matrix is analyzed by comparing the values of clusters of measures. For the analysis that follows, the reader is referred to Figure 2 for a schematic presentation of the clusters, and to Table I for the obtained values of the measures of the dependent variables used in this study.

The first step in the analysis is to establish some evidence that convergent validity is occurring. The correlations of the measures of the same trait by different methods (the "validity diagonal" in Figure 2) 


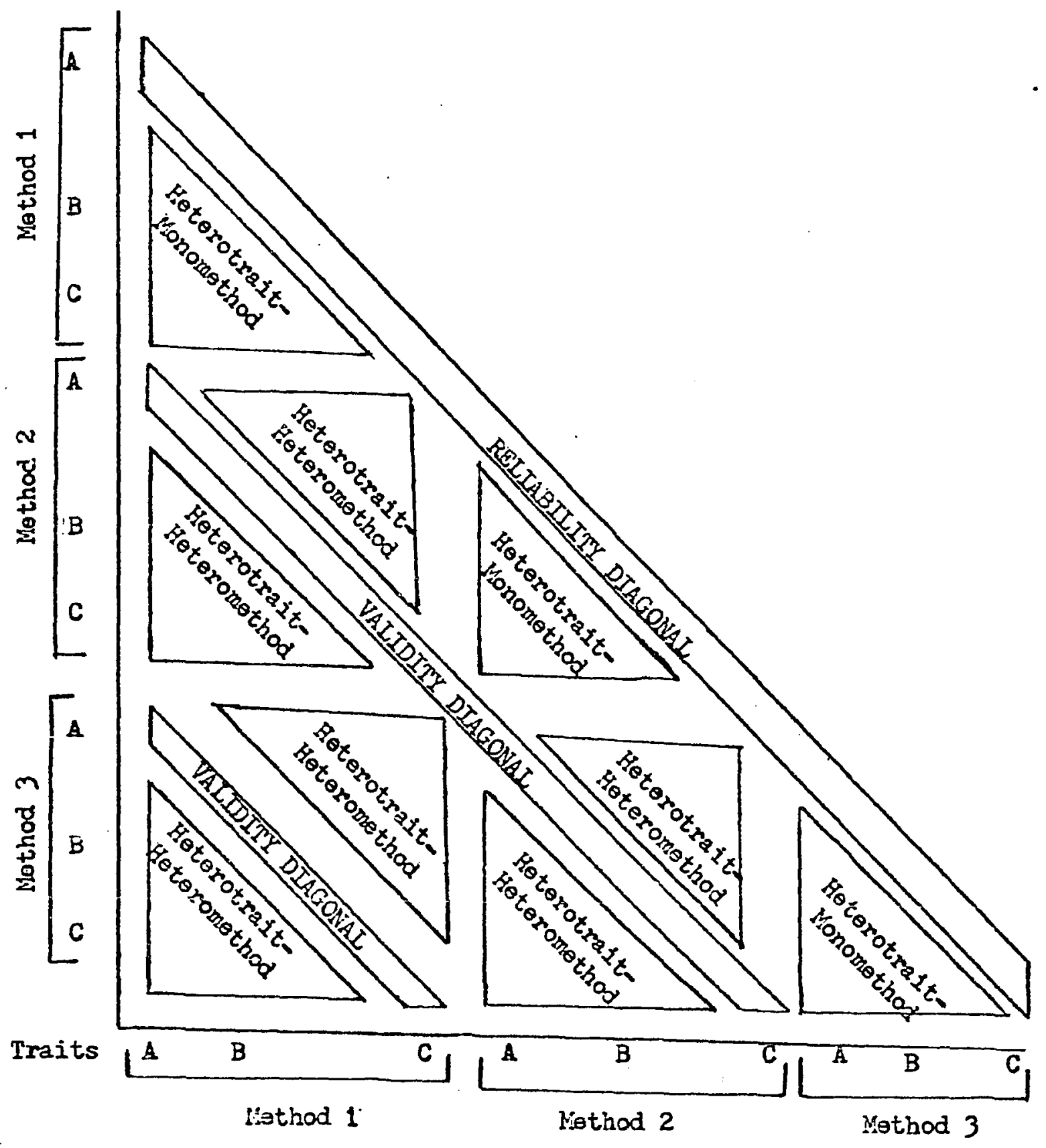

Flgura 2. Scheñatic Design of a cultitrait iultimathod liatrix. 
TABLE I

MAIRIX OF CORRELATIONS: DEPERDENT VARLABLES

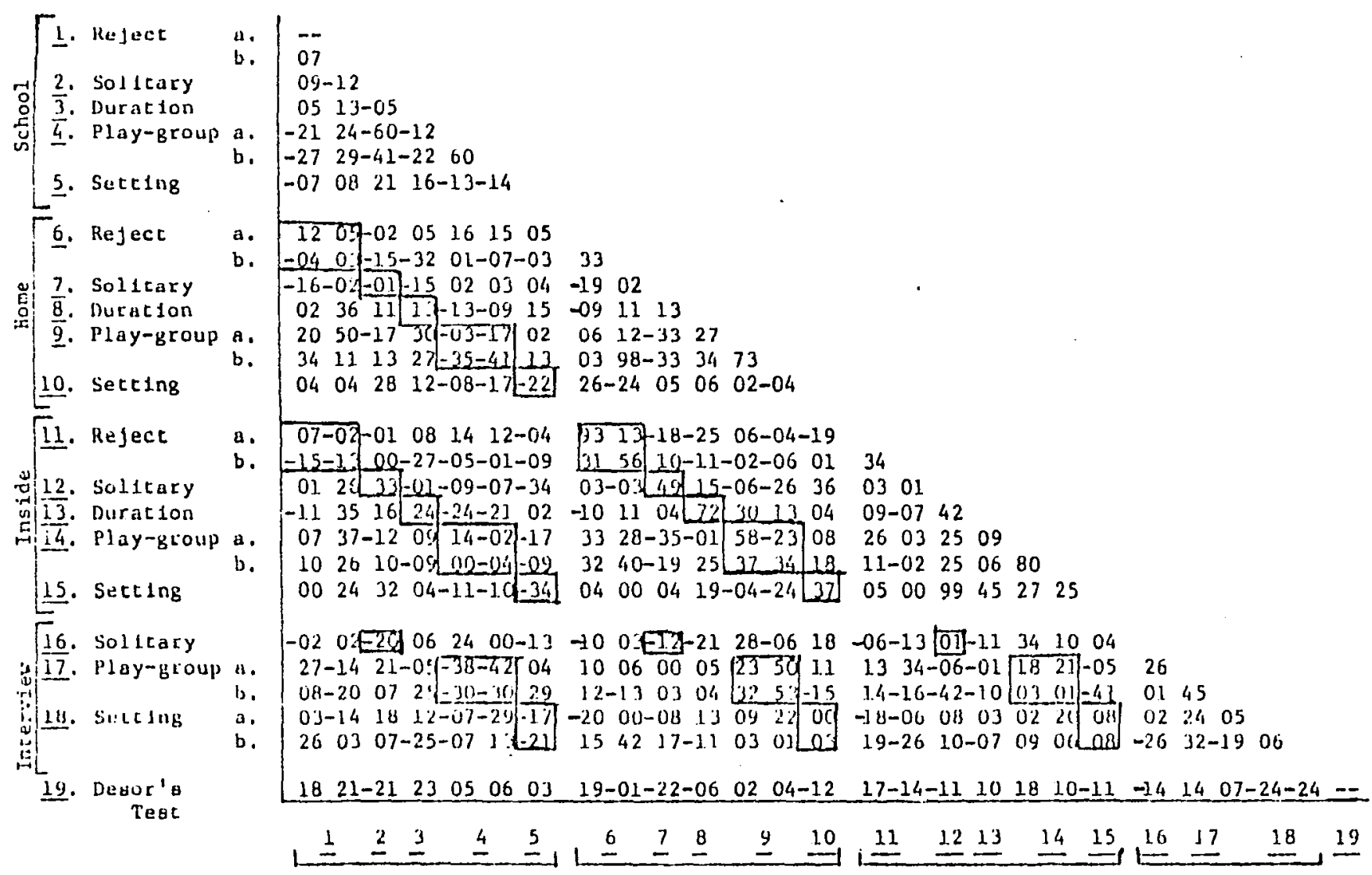


should be significantly different from zero and large enough to encourage further evaluation. In analyzing the validity diagonals in Table $I$, note that the diagonal is sometimes two cells wide (because some trait-method units are composed of two measures), and that the diagonals in the fourth method-block (the "Interview" rows) are not continuous (because the interview method was not used for all traits). The significance level for a correlation is a function of the sample-size from which the statistic was derived, as can be seen from the formula for arriving at the appropriate value of $t:^{3}$

$$
t=\frac{r \sqrt{n-2}}{\sqrt{1-r^{2}}}
$$

For a significance level of .05 and a sample-size of 32 , $\underline{r}$ is significant at 0.349 . Since Table I reports the correlation coefficient only to two significant places, a correlation of 0.35 or greater is necessary to be considered significantly different from zero.

An examination of the validity diagonals in Table I reveals three things. First, there is evidence of convergent validity between measures of traits taken from the HOME method and the INSIDE method. This was to be expected, since the measures observations inside the dwelling are in fact a subset of all observations made in the housing environment. This should lead to some autocorrelation, and would inflate the value of the coefficient of correlation (relative to other coefficients of truly independent variables). Considering the problem of autocorrelation, the values in the validity diagonal are fairly low, with the exception of the measures of setting and of duration. Second, in the remaining validity diagonals, the only significant values are between the play-group measures, 
and even those values are fairly low. Third, in the diagonal between the SCHOOL method and the INSIDE method, the values for the play-group measures are not significant.

Taken together, these three points suggest that, with the exception of size of piay-group, the 'traits' measured in this study are not internally consistent. The only evidence of convergent validity of traits is a moderate relationship between a subset and the group from which it was drawn; and the subset fails to show even the convergent validity with the play-group measures from the SCHOOL method that is shown by the HOME method. There are three possible explanations for this failure to establish convergent validity. It is possible that all but one method failed to "really" measure the trait. This is tantamount to saying that one set of diagonals is of a different class than the others. This would be likely to show up elsewhere in the matrix. If the pattern of off-diagonal (heterotrait-heteromethod) values are widely divergent between one method block and the others, this position would be supported. Even though such a pattern does not occur in Table $I$, there is no way of definitively ruling out the possibility that only one method is "really" measuring the trait. A second possible explanation is that none of the methods is a measure of the traits. A third possibility is that the trait is not a functional unity. These possibilities can be evaluated later in the study: if oniy one method yields significant results, the first possibility would be supported. If all the methods yield some significant results, but without any pattern, the last possibility would be supported. If no significant results are found, the possible alternate explanation would remain that the "real" traits were never measured.

Note also the low values in the valididty diagonals for the INTER- 
VIEW method. Even for behavior in the home setting, what the parent or child says is occurring is not very highly related to what was observed In that environment. The only exception was the number of people in the play-group. The parents' estimates of their children's solitary play were particularly poor.

A second step in the analysis is to establish that the measures of convergent validity discriminate from measures of purportedly different traits. This means that the values in the validity diagonals should be larger than the values of the other traits measured by that method (the heterotrait-heteromethod values--the triangles of values on either side of the validity diagonal). In other words, the validity values should be higher than similar values for measures having neither the trait nor the method in common.

An examination of Table I reveals two things. First, there is a fairly consistent pattern in the significant off-diagonal values and the second measure of the trait, Rejecting Interaction (a), the measure, "ignoring interaction.". It correlates particularly with duration of play in other observed settings and with the proportion of peers in the playgroup inside the dwelling unit. Note that this pattern does not occur for the measures of the trait, "Play-Group," when the INTERVIEW method was used. This suggests that there is a mild tendency that the longer a child plays at one thing or the more children he is playing with, the more likely a child is to ignore other children during that time. The second thing is that there are several significant off-diagonal values that follow no pattern. of these, only one is a possible threat to convergent validity, since only one is related to a significant diagonal value. That one, the relation between the number of settings used in the 
home environment and the percent of time inside the dwelling in solitary play, is a lower value than the corresponding validity value, percent of time in solitary play both inside the dwelling and in the home environment.

The third step in the analysis is to be sure that the validity value is not spurious due to some common effect of the method of obtaining the measures. This means that the values in the validity diagonal should be larger than the values of the correlations of the trait with other traits measured by the same method (the heterotrait-monomethod values). In other words, the values for a trait should be closer for other methods for measuring the same trait than when compared to the same method for measuring other traits.

An examination of Table I shows three things. First, there is a consistent effect, across all methods, of a moderate to strong relationship between both measures of the trait, "Play-Group." The values range from a high of 0.80 to a low of 0.45 . In other words, the two measures of the size of the play-group measure roughly the same thing. Considering that the second measure, the number of peers in the group, is more frequently represented in the validity diagonals by significant values, it seems $11 k e l y$ to be the dominant measure in testing the hypotheses. Second, there is a consistent lack of relationship, across all methods, between the two measures of the 'trait,' "Rejecting Interaction." The values range from a low of 0.07 to a high of 0.34 . In other words, the two measures do not measure the same thing but are two distinct components. Considering that the second measure, "ignoring interaction," is significantly correlated with measures of other traits, the more useful measure will probably be the first one, "refusing overtures for interac- 
tion," since it is independent of all the other measures. Third, there are several measures in the heterotrait-monomethod triangles that are interrelated. The number and percent of pears in the play-group in the school environment is significantly negatively related to the percent of time there which was spent in solitary play. The more a child plays alone, the fewer peers he plays with. Note that this relationship does not hold elsewhere (although the pattern recurs in observation in the home environment), suggesting that the effect may be due to the group-pressure of the school situation: some children are popular and are included in many children's play-groups; others are not popular, and play alone or with a few friends. This is not to say that popularity caused the high peer-interaction, or that the high peer-interaction caused the popularity, but only that, observing the situation already in progress (and presumably in some sort of equilibrium), the two go together. There are also several correlated measures inside the dwelling environment. Percent of time in solitary play correlates highly with the duraition of play and the number of settings used (these two are also correlated). In other words, inside the dwelling unit playing alone is an absorbing and wide-ranging activity for young children. Note that this relationship is not suggested at all in the other methods.

The last step in the analysis is to check for the same patterning among the variables in all the clusters. Actually, this has been done throughout the first three steps. Consistently, patterns may be found that are common to observation in the day-care setting and observation in the home setting, but which are less strong in observation inside the dwelling unit.

In summary, it can be seen that there is little evidence for con- 
vergent validity of the measures of the traits used in this study. The only measures that show clear (but moderate) signs of convergent validity. are those which deals with the size of the play-group. Of these measures, the stronger of the two is the measure of the number of peers in the playgroup, although the differences are minor. It can also be seen that there is some clear clustering of measures. Ignoring interaction, duration of activity, and percent of peers in the play-group tend to be related across settings. This suggests that the division of traits into variables may be inaccurate: these three measures may constitute a trait that reflects the optimization of group interaction. The same could be said of the number of settings, duration of activity, and time in solitary play inside the dwelling unit. And in the school setting, solitary play is inversely related to peer-group interaction.

\section{MATRIX OF CORREIAATIONS: INDEPENDENT VARIABLES}

The multitrait-multimethod matrix is not entirely suitable for the analysis of the measures of the independent variables used in this study. It will be recalled that, to have a multitrait-multimethod matrix, one must employ more than one trait and more than one method. While there are multiple measures among the independent variables, only one method was used to obtain the measures. As a result, convergent validity of traits cannot be established, although discriminant validity may still be examined in Table II. There will be two steps to this analysis.

An examination of the autocorrelation triangles (the correlation of measures of the same variable) shows that the measures of each dimension are highly intercorrelated. Although all but one of the measures of the 
TABLE II

MATRIX OF CORRELATIONS: INDEPENDENT VARIABLES
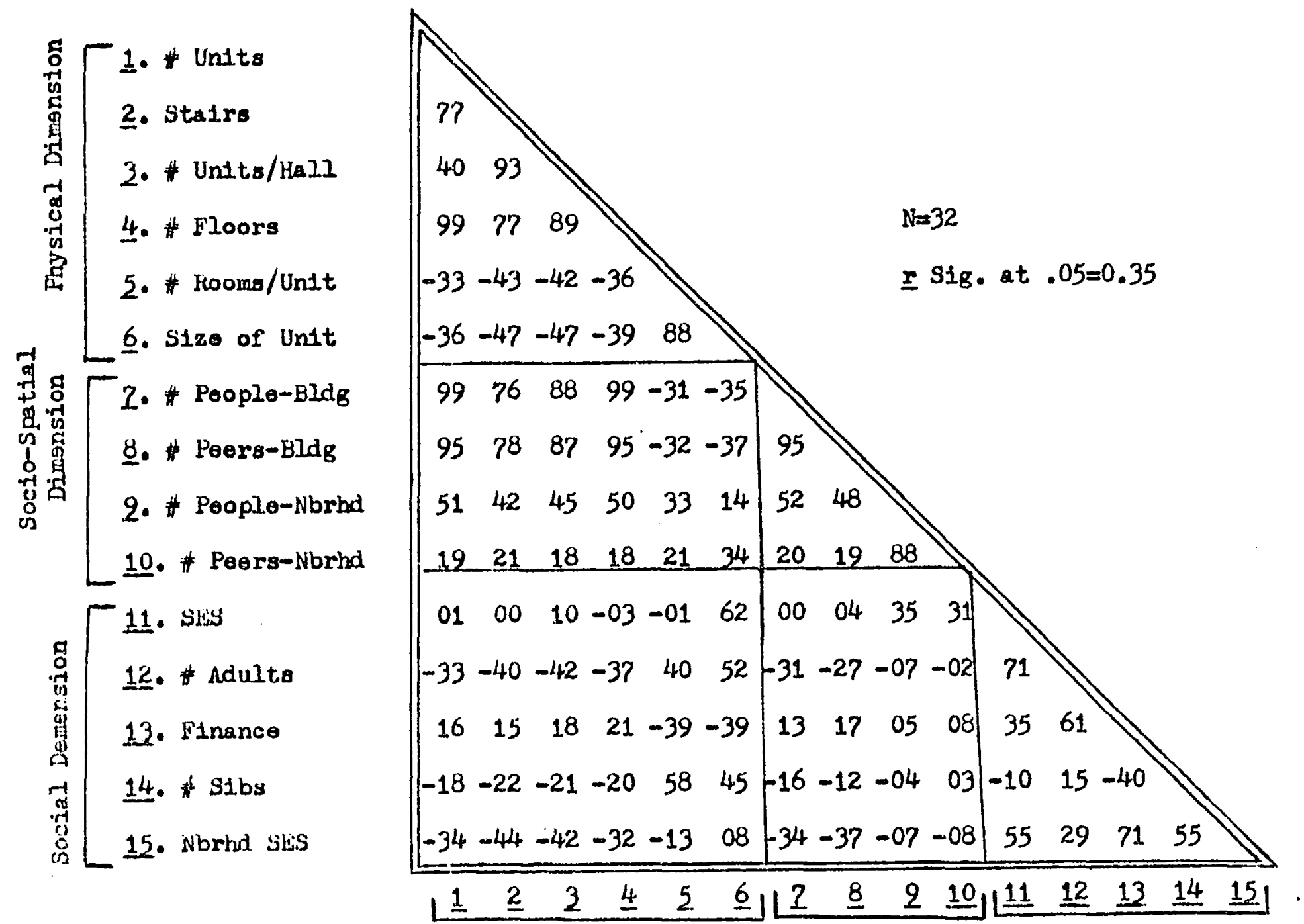
physical dimension are significantly correlated, the measures still fall clearly into two groups. The first four (number of units in the building, presence of an inside stairway, units per hall, and number of floors) are all descriptive of the type of building and are highly intercorrelated. The next two (number of rooms and size of unit) are descriptive of the dwelling unit, and are both highly correlated and exhibit similar patterns of low correlation with the measures of the building-type. Similarly, the measures of the socio-spatial dimension also fall into two groups. The first two neasures (number of people and number of peers in the building) are descriptive of the population in the immediate environs of the dwel1ing unit, and are also highly correlated. The number of people in the child's range is mildly correlated with the measures of the population in the building, also. Finally, the measures of the social dimension show a multidimensional grouping. The number of adults caring for the child is strongly related to social class and to financial ranking (which is also related to social class), and appears to be descriptive of the childcare characteristics of the family. The number of siblings is positively related to neighborhood social class and inversely related to financial rank (which is also positively related to neighborhood social class), and neighborhood social class is also correlated with family social class. This grouping appears to be descriptive of family social class.

An examination of the rectangles containing the correlations between the variables throws more light on the groupings. There are very high correlations between the first group of measures of the physical dimension (descriptive of the building type) and the first group of measures of the socio-spatial dimension (descriptive of the building population). Further, both groups follow similar patterns in their relationship to the social 
dimension. While some relationship between the physical and socio-spatial dimension was expected, these relationships are so great that they lead inevitably to the conclusion that the measures which are descriptive of the building population act as if they belong to the physical dimension. The measures which are descriptive of population in the immediate environs of the child's dwelling unit, however, while correlated to the measures of physical dimension, are only moderately correlated and they do not follow a similar pattern in relation to the social dimension. This suggests that the socio-spatial dimension is more distinguishably measured by the descriptors of the neighborhood population. Finally, the high correlation between the number of peers and the total number of people in both the building and the child's neighborhood suggest that, even if there were a difference in effect between contact-potential (number of people) and interaction-potential (numbers of peers), it probably will not be discovered in this study, or will at best appear as a very small difference, due to the high interrelationship of those measures.

\section{SUMMARY STATISTICS: DWELLING-TYPE}

Throughout the discussion in the previous chapters, the problems were considered on two levels: the effect of dwelling-type (single-family vs. multi-family dwellings) on behavior and the effect of the communication-facilitating characteristics of the housing environment. It remains to be seen which of the characteristics of the housing environment are distinguishable on the basis of dwelling-type. The mean values of each characteristic for single-family dwellings and multi-family dwellIngs, and the significance of the differences between the means, are pre- 
sented in Table III.

The variable, "Dwelling Type," clearly distinguishes between measures of the physical dimension of the housing environment and the population of the building. But it does not distinguish between measures of . the socio-spatial dimension of the housing environment, i.e., the popula-. tion in the immediate environs of the housing unit. In other words, the neighborhood environments observed in this study were similar, which suggests that multi-family and single-family dwellings must have been present in the neighborhoods of both groups. There were no neighborhoods composed entirely of apartment blocks. This means that, whatever the results which are obtained in analyzing the hypotheses, they carry with them the restriction that they cannot be generalized to housing environments in which the entire neighborhood is composed of either low-density singlefamily dwellings or high-density multi-family dwellings. The results will not necessarily apply in Midtown Manhattan. Further, it will be possible in this study to find an effect due to the housing environment (for instance, due to the number of peers in the neighborhood) which does not bear on the original question of what effect apartment living has on children.

There are a few other differences in the social dimension. The childcare characteristics are different between the two groups. Apartment families are more likely to be single-parent families, and the source of income is more likely to be from hourly wages rather than a monthly salary. There were also significantly more girls among the apartment families. Finally, apartment families were more mobile. They had lived in their present unit significantly less time than the families in singlefamily dwellings, although there was no difference in the time the fam- 
TABLE III

SUTMARY STETISTICS OF INDEPSWDENT VARIABLES

BY DWELITNG TYPE

\begin{tabular}{|c|c|c|c|c|}
\hline \multirow[b]{2}{*}{ VARIABIE } & \multirow[b]{2}{*}{ MEASURE } & \multicolumn{2}{|c|}{ MFATS } & \multirow[b]{2}{*}{$\begin{array}{c}t- \\
\text { VALUE }\end{array}$} \\
\hline & & $\begin{array}{l}\text { SINGLE- } \\
\text { FAMIIYY }\end{array}$ & $\begin{array}{l}\text { IUUTI- } \\
\text { FAMIIIY }\end{array}$ & \\
\hline \multirow{2}{*}{\multicolumn{2}{|c|}{$\begin{array}{l}\text { Physical Dimension: } \\
\text { Dwelling Unit \#Room/Unit } \\
\text { Socio-Spatial Dimensions } \\
\text { Bldg Fopulation \#People/Bldg } \\
\text { NPeiers/Bldg } \\
\text { Norid Population \#Pople/Nbrind } \\
\text { Aocial Dimension: }\end{array}$}} & $\begin{array}{r}7.75 \\
20808 \\
\end{array}$ & $\begin{array}{r}3.69 \\
827.5 \\
\end{array}$ & $\begin{array}{l}5.064^{*} \\
5.171^{*}\end{array}$ \\
\hline & & $\begin{array}{r}4.38 \\
=1.94 \\
100.8 \\
17.06\end{array}$ & $\begin{array}{l}124.5 \\
10.63 \\
152.3 \\
15.44\end{array}$ & $\begin{array}{l}2.894^{*} \\
3.720^{*} \\
0.713 \\
0.262\end{array}$ \\
\hline \multirow{2}{*}{\multicolumn{2}{|c|}{ 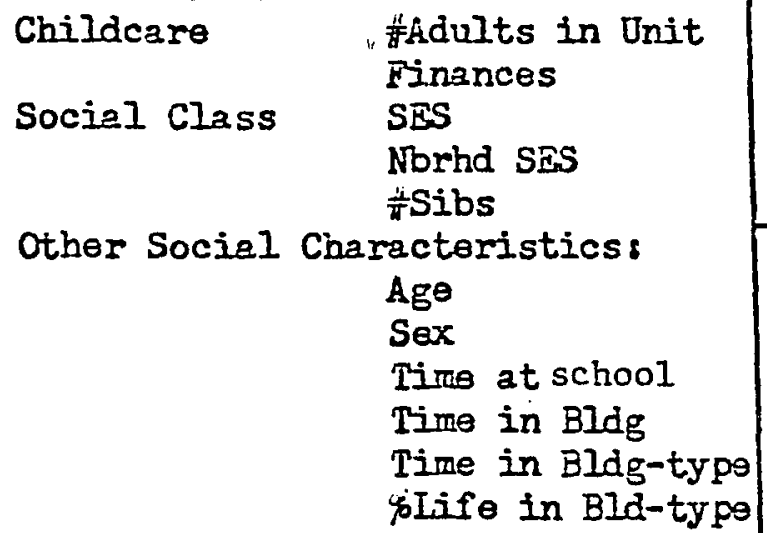 }} & $\begin{array}{l}1.94 \\
4.25 \\
2.69 \\
2.13 \\
2.19 \\
\end{array}$ & $\begin{array}{l}1.31 \\
5.25 \\
3.06 \\
1.69 \\
1.63 \\
\end{array}$ & $\begin{array}{l}3.834^{*} \\
3.464^{*} \\
0.853 \\
1.031 \\
1.490\end{array}$ \\
\hline & & $\begin{array}{r}62.3 \\
1.25 \\
15.0 \\
27.69 \\
47.81 \\
74.8\end{array}$ & $\begin{array}{r}58.0 \\
1.69 \\
14.1 \\
10.69 \\
37.94 \\
64.1\end{array}$ & $\begin{array}{l}1.829 \\
2.671^{*} \\
0.259 \\
2.671^{*} \\
1.324 \\
0.909\end{array}$ \\
\hline
\end{tabular}

* Sig. at .05 level 
ilies had been in their current building-type, nor was there a difference in the percent of the child's life spent in the current building-type. Before going on to the next section, the range of dwelling-types actually obtained in this study should be mentioned. Surprisingly, a fairly broad spectrum of the possible types was obtained. There were no children living on one-acre wooded lots. Four children lived in the same 16-story tower. The rest of the children from multi-family dwellings lived in structures from one to five stories high. The structures were scattered throughout the city, including several in the suburbs. Eight of them lived in the downtown area near the university. No children from single-family dwellings lived downtown. Several lived in the suburbs. Like the children from multi-family dwellings, most of the children from single-family dwellings lived inside the city.

\section{QUALITATIVE ANALYSIS OF MEASURES}

The purpose of the preceding quantitative analysis was to evaluate the measures and their relation to each other. This understanding can be further developed by considering information gathered in the process of collecting the quantitative data.

A constant question in any observational study is, "How much is the Observer's presence interfering with the 'normal' activity of the Subjects?" There is no way to answer this directly, lacking an inside informant (preferably an omniscient inside informant). Indirect evidence for this question can be gleaned from the Subjects' reactions directed toward the observer. The evidence for this study is mixed. Almost every child, at one time or another, did acknowledge the Observer's presence, 
either by showing him the latest creation in the craft shop, or by asking for help with a shoelace or a zipper. For the most part, however, he was ignored. Toward the end of the period of observation at the day-care center, the parent of a prospective student was being shown a classroom in which the Observer was recording. The parent was introduced to the teacher, who then introduced the parent and the child to the aides and to the other children. The teacher then started a conversation with the parent, only to have it interrupted by the question, "Is he an aide, too?" The teacher turned, realizing the observer was present, and then introduced him, "I'm sorry. He's observing the children for a project, and I forget. he's even here." As far as the observer could tell, this was a common reaction for most teachers and children. Further evidence for this assertion can be found in the protocols, which include not a few 'naughty' behaviors. For a few children, however, the observer's presence continued to be noted from the first day to the last. One little girl greeted the Observer every day with, "Get out. I don't want you here" (she would then let the observer stay, having made her presence known). Another child viewed the observer with obvious suspicion, and would glance nervously at him whenever she was talking to anyone else in his presence. These were the only individuals for whom such evidence was noted regularly. Another source of concern about the measures used in this study is Desor's test. It had to be adapted for use with children, and the question occurs of how well such young children were able to deal with it. Although four children failed to perform adequately on the test, only one appeared to be unable to comprehend it. The other three simply refused to do what was asked: one, because she was more interested in teasing the Investigator (the same one who told the observer to get out of the 
classroom); the other two, because they were more interested in playing with the configurations of a full room (rearranging what little free space remained). That the children understood the test apparatus as a room and children was confirmed on later visits. Frequently, the children would ask if the Investigator brought his "toy," and asked to play with it. The play always involved people using a room, frequently focusing on coming in and going out of the room. It is interesting to note that at least five children asked why the door drawn on the back wall didn't open (one child offered to get her scissors and fix it so it would open), and the children's play focused on getting into a room whose door would not open. A few children also asked why the room had no ceiling, although none questioned the missing wall. It should also be noted that the children did not attend to the height of the figures in relation to either the apparent age of the characters or the size of the room. When they needed an adult, one of the figures was designated as an adult. Further, when it was apparent that some figures were adults and some were children, the space between the figures did not appear to be different. The children are used to playing with dollhouses and Barbie and Ken dolls, all of which represent adults in adult-scale environments: it could be that by age 4 children are already conditioned to projecting make-believe situations on an adult scale. If an effort were to be made to standardize Desor's test, this would be a dimension worth evaluating.

A third concern is to determine what was lost by quantifying the protocols. Since there are an indefinite number of perspectives on any given position, there were probably an indefinite number of things lost. Three were noted in the process of observing. First, the specific quan- tifications lost the Individual child's 'style,' his typical way of doing 
things. This was noted especially in the way the children handled anger. One boy, rather big for his age, would 1iterally throw himself into a corner when he got angry and hold onto the walls to keep from hitting the child he was angry with. Another boy used rather complicated verbal ski11s. On one particularly striking occasion, he was upset because the aide wanted him to clean up a mess that he felt he shouldn't clean up a1one. He insulted the aide, and then ran outside. By the time he was returned to his room, he had found someone to help him clean up. When his friend got tired of it and left, he continued to work at it until the aide's attention was absorbed elsewhere, and then he left also. A third child bawled and threw things. When he was all cried out, he sat quietly for a minute, and then said to the aide, "There, now isn't that better?" A fourth sat in a chair and whimpered. The failure to quantify these differences is, to some extent, an intrinsic failing of quantification: were the range of these styles of dealing with anger known, a scale could have been devised and they could have been quantified.

But every quantification will always be limited to a) the parameters known, and b) only as many of those parameters as can be reasonably dealt with, given limitations of data and mathematical skill. Even someone as skilled as Jay Forrester could include only 17 variables in his analysis of urban dynamics, 4 and even then he manipulated no more than seven at once. Second, the depth and strength of the interactions were not measured. Some children seemed to flit from thing to thing, some to work slowly and steadily on one thing. Some had a few friends whom they played with for long periods of time and regularly; others simply had few friends and played alone. Some played in packs, not really interacting with any child in particular; others controlled the pack and maintained strong re- 
lations with each child in the pack. This dimension is present in the measures used--the number of settings used, the duration of play, the size of the play-group--but is not collected into one measure. Third, the observation of the depth of interaction led to the realization that different children used different methods for social control. Two little girls were especially adept at threatening to withdraw their friendship from the offenders. Several little boys used threats of violence. Other children used insults and name-calling. Further, not all the children exercised these forms of control; there were obvious peer-group leaders. This dimension is not included in the analysis.

Fina11y, while discussing the study with the parents, suggestions were offered for other variables in the housing environment that might Influence the children's behavior. In any event, the parents found them to be sources of Irritation. The most frequently mentioned complaint of apartment-dwellers was the lack of acoustical privacy. They were concerned either that their noise was disturbing the others in the building (hence, they tried to restrain their children's exuberance) or they were concerned that their neighbor's noise was disturbing them and their children. This dimension was not considered in this study, except insofar as it may be a correlate of the dwelling-type dichotomy. Note, in this regard, Mitchell's study, 5 referred to earlier, which pointed out that families in Hong Kong are able to adjust to restrictions on privacy that come from relatives, but not when they come from unrelated individuals. This would suggest that poor acoustical insulation in a single-family dwelling is less problematic than poor acoustical insulation in an apartment. A second complaint was that the child was restricted in range because of high amounts of traffic on the streets. Interestingly, people 
in single-family homes on busy streets did not mention this. Further, other apartment-dwelling parents said they felt they trusted their children beyond their limits (because of the difficulty of surveillance from the apartment), rather than restricting the child. In any event, some . measure of the location of busy streets might prove useful. Third, a common complaint was the lack of adequate play-space for the children. This is probably the best-publicized of the complaints about apaitmentliving for children, and in fact two of the recently built structures had provided specific play-areas for the children.

\section{SUMRARY}

To summarize this chapter, three things may be said about the quality of the measures used:

First, while the measures of the dependent variable have high face validity, i.e., by their definitions they should be valid measures, convergent validity between sinilar measures could not be established. While any of three alternatives may. explain such a failure, the high face validity suggests that the lack of convergent validity is due to the absence of a unified trait, that perhaps there is no consistency in behavior across the situations which were observed. This possibility will be explored further in the next chapter.

Second, the measures of the independent variables seemed to fall into five groups of highly intercorrelated measures. The intercorrelations were so high that if an effect were found with one measure, it could be expected to be found with the others as well. It should be noted that the number of peers in the building and the number of peers in the child's neighborhood were not highly intercorrelated, and both are key measures. 
The first is a key measure of the socio-spatial dimension which is highly correlated with the physical dimension of the housing environment, and the second is a key measure of the socio-spatial dimension only. These two measures are also the key independent measures that will be used for * analyzing the hypotheses in the next chapter.

Finally it was seen that the dwelling-type dichotomy basically distinguishes between characteristics of the physical dimension of the housing environment. It also carries with it some distinctions in the characteristics of the social dimension, the most interesting one being that people who live in apartments tend to move around more. Thus, it seems that the distinction between multi-fanily and single-family dwellings does have a relation to the communication-characteristics of the housing environment, at least in regard to the building-population characteristics. 
NOTES

1. Pierrefeu, quoted in Simone de Beauvoir, The Ethics of Ambiquity, tr. B. Frechtman, New York: Citadel, 1948, p. 129.

2. Donald T. Campbell \& Donald W. Fiske, "Convergent and discriminant validation by the multitrait-multimethod matrix," Psychological Bulletin, 1959, 56, 81-105, p. 100.

3. W.L. Hays, Basic Statistics, Belmont, Cal.: Brooks-Cole, 1967, P. 661 . 1970 .

4. Jay W. Forrester, Urban Dynamics, Cambridge, Mass: MIT Press,

5. Robert E. Mitche11, "Some social iriplications of high density housing," American Sociological Review, 1971, 36, 18-29. 


\section{RESULTS: ANALYSIS OF HYPOTHESES}

Art and Science do not establish themselves despite failure but through it.1

\section{COGNITIVE STRUCTURING}

The first hypothesis is concerned with the strategy of cognitive structuring. It states:

1) Children living in housing environments with greater num- bers of people show greater acceptance of social density than children living in housing environments with fewer people.

It will be recalled that the specification of the contact-potential of the housing environment (number of people) was due to the assumption that asymmetric or one-way contacts (i.e., an adult giving orders to a child) are as important for sucial density as symmetric contacts (interaction). The measure of the dependent variable was the child's score on Desor's test. There were two measures of the independent variable, the number of people in the building and the number of pecple in the child's range (neighborhood); and there were two alternate measures, the number of peers in the building and the number of peers in the child's range. The assumption that the contact characteristics are more important can thus be examined as well.

The correlation between Desor's test and the measures of the communication-factlitation characteristics of the housing environment are presented in Table IV. The only significant* values were for the measures 
of the building environment.

The hypothesis is supported by the data, in a qualified fashion. In the first place, only the immediate environment-the population characteristics of the building itself--is related with cognitive structuring. Since the measures of the population of the building are correlated so highly with the measures of the physical dimensions of the housing environment, it is not clear at this point whether the effect is due to the physical dimension of the building-environment, or to the socio-spatial dimension (the populations characteristics) that are based on it. In any event, it is clear that the characteristics of the child's neighborhood do not have a relationship to tolerance for density. Perhaps, were the neighborhood characteristics more distinct between apartment and singlefamily dwellings, some relationship would be found; but this study suggests that, should such a relationship occur, it would be due to the correlation with the effect of the building. Second, although the differences between the significant measures are too small for clear interpretation, the direction of the differences suggests that either the distinction between contact and interaction is not necessary for these children, or else interaction-potential is more important than contact-potential for cognitive structuring. What is supported, however, is that children living in buildings with more peers show a greater tolerance for so-

* Significance is only reported at the .05 level, no matter how large the F-value. It should be understood as "at least significant at the .05 level." The purpose of reporting significance levels here is to test against the alternate hypothesis that the statistic could be the result of chance fluctuations. Since the probability of any discrete outcome is infinitesmal, one must pred ${ }_{2}$ t a realm of outcomes and combine their probabilities. To change significance levels after examining the size of the F-value is to invalidate this procedure. 
TABLE IV

CORRELATIONS : COGNITIVE STRUCTURING AND COMMUNICATION CHARACTERISTICS

\begin{tabular}{|c|c|c|}
\hline \multirow{2}{*}{ \#Peers: } & & $\begin{array}{l}\text { Desor's } \\
\text { Test }\end{array}$ \\
\hline & $\begin{array}{l}\text { Bldg } \\
\text { Range }\end{array}$ & $\begin{array}{l}0.634 * \\
0.071\end{array}$ \\
\hline People & $\begin{array}{l}\text { Bldg } \\
\text { Range }\end{array}$ & $\begin{array}{l}0.611 * \\
0.223\end{array}$ \\
\hline
\end{tabular}

* Sig. at .05 level 
cial density than children living in buildings with fewer peers.

\section{WITHDRAWAL FROM INTERACTION}

The second set of hypotheses are concerned with withdrawal from interaction as a strategy for responding to the housing environment. The two hypotheses will be considered in turn. The first states:

2a) Children living in housing environments with a greater number of peers reject overtures for interaction more frequently than children living in housing environments with fewer peers.

This hypothesis specifies measures of the interaction-potential of the housing environment, although again the alternate measures, those of contact-potential, will also be considered. The dependent variable, rejection of overtures for interaction, is defined in two ways, by refusal of interaction and by ignoring interaction. Each of these operational definitions is measured in three situations: the day-care center (SCHOOL), the home, and inside the dwelling. It will be recalled that the subset of observations, INSIDE, was analyzed separately to account for the possibility that, since the observations at the day-care center were done only inside, the subset of observations made inside the home would be more comparable.

The correlation between the population characteristics of the building and refusing overtures for interaction are presented in Table V. Significant values were found for the relation between the population characteristics of the child's range and behavior in that environment. The correlation between the population characteristics of the building and ignoring interaction are presented in Table VI. All the measures of the communication-characteristics of the housing environment correlated signifi- 
cantly with behavior in that environment, although the number of people in the neighborhood was most highly correlated.

The hypothesis, then, is strongly supported, although the pattern underlying that support is a complex one. To begin with, the relation . of the housing environment with refusing overtures for interaction occurs only in the housing environment itself. It does not generalize to the other environment studied. All of the sets of measures follow this pattern. Second, only the communication-characteristics of the child's range are related to refusing interaction (Table V). Within that limitation, the interaction-potential (number of peers) is clearly the stronger correlate when considering behavior in the home environment, although that difference is much less when only behaviors occurring inside the dwelling are considered. Third, ignoring interaction is best predicted by the contact-potential of the child's neighborhood (Table VI), although all the measures of the home situation correlate significantly with ignoring interaction. Restating the original hypothesis, it can be said that children living in neighborhoods with more peers are more likely to reject interaction, and children living in neighborhoods with more people are more likely to ignore interaction than children living in neighborhoods with lower cormunication-potentials.

The second hypothesis dealing with the strategy of withdrawal from interaction states:

2b) The relationship between the number of peers in the housing environment and solitary play is described by an inverted-U function: at both very low and very high numbers of peers, solitary play occurs more frequently.

Again, both the specified measures of communication potential (number of 
TABLE V

CORRELATIONS: REFUSING INTERACTION

AND COMMUNICATION CHARACTERISTICS

\begin{tabular}{|c|c|c|c|}
\hline \multirow{2}{*}{ 非Peers: } & \multicolumn{3}{|c|}{ Refusing Interaction } \\
\hline & School & Home & Inside \\
\hline B1dg & 0.073 & -0.039 & 0.049 \\
\hline Range & -0.029 & $0.691 *$ & 0.887 \\
\hline BIdg & 0.063 & -0.114 & 0.057 \\
\hline Range & 0.00 & $0.478 *$ & $0.821 *$ \\
\hline
\end{tabular}

TABLE VI

CORRELATIONS: IGNORING INTERACTION AND COMMUNICATION CHARACTERISTICS

Ignoring Interaction

\begin{tabular}{|c|c|c|c|}
\hline & School & Home & Inside \\
\hline $\begin{array}{l}\text { HPeers: } \\
\qquad \text { Bldg }\end{array}$ & & & \\
\hline Range & & $0.471 *$ & $0.435 *$ \\
\hline $\begin{array}{l}\text { BIdg } \\
\text { Range }\end{array}$ & $\begin{array}{r}0.069 \\
-0.151\end{array}$ & $\begin{array}{l}0.493 * \\
0.587 *\end{array}$ & $\begin{array}{l}0.517 * \\
0.615 *\end{array}$ \\
\hline
\end{tabular}

* Sig. at .05 leve1 
peers) and the alternate (number of people) will be considered. The dependent variable, solitary play, has four measures: the parent's esti-mate of time spent in solitary play and the percent of the time in the protocols spent in solitary play for each of the three situations: the . day-care center, the home, and inside the dwelling.

To test this hypothesis, a curve-fitting procedure, described earlier, was employed. The procedure results in two statistics per measure: the zero-order correlation between the measure of communication potential and the measure of solitary play, and the first-order regression coefficient which enters the squared value of the measure of solitary play. The values for these statistics are presented in Table VII. None of them is significant.

There is no evidence to support hypothesis $2 b$, either as stated or in a simpler, linear model for the relationship.

\section{FILTERING POTENTTIAL INTERACTION}

The third set of hypotheses are concerned with filtering potential interaction as a strategy for responding to the housing environment. The three hypotheses will be considered in turn. The first is:

3a) Children living in housing environments with a greater number of peers allocate less time to each behavior episode than children living in housing environments with fewer peers.

As before, measures of both the interaction-potential (specified in the hypothesis) and measures of the contact-potential will be compared. The dependent variable is measured by the average duration of behavior episodes in each of the three situations: the day-care center, the home, and inside the dwelling. 
TABLE VII

CURVE FITTING: SOLITARY PLAY AND COMMUNICATION CHARACTERISTICS

\begin{tabular}{|c|c|c|c|c|c|c|c|c|c|}
\hline & \multicolumn{6}{|c|}{$\%$ Solitary } & \multirow{2}{*}{\multicolumn{2}{|c|}{$\begin{array}{l}\text { Parent's } \\
\text { Estimate }\end{array}$}} \\
\hline & & \multicolumn{2}{|c|}{ School } & \multicolumn{2}{|c|}{ Home } & \multicolumn{2}{|c|}{ Instde } & & \\
\hline & & $\begin{array}{l}\text { zero } \\
\text { order }\end{array}$ & $\begin{array}{l}\text { first- } \\
\text { order }\end{array}$ & $\begin{array}{l}\text { zero- } \\
\text { order }\end{array}$ & $\begin{array}{l}\text { first- } \\
\text { order }\end{array}$ & $\begin{array}{l}\text { zero- } \\
\text { order }\end{array}$ & $\begin{array}{l}\text { first- } \\
\text { order }\end{array}$ & $\begin{array}{l}\text { zero- } \\
\text { order }\end{array}$ & $\begin{array}{l}\text { first- } \\
\text { order }\end{array}$ \\
\hline \#eers & $\begin{array}{l}\text { Bldg } \\
\text { Range }\end{array}$ & $\begin{array}{l}0.258 \\
0.130\end{array}$ & $\begin{array}{l}0.346 \\
0.130\end{array}$ & $\begin{array}{l}0.155 \\
0.099\end{array}$ & $\begin{array}{l}0.155 \\
0.099\end{array}$ & $\begin{array}{l}0.124 \\
0.111\end{array}$ & $\begin{array}{l}0.124 \\
0.177\end{array}$ & $\begin{array}{r}0.074 \\
-0.203\end{array}$ & $\begin{array}{l}0.080 \\
0.203\end{array}$ \\
\hline \#People: & $\begin{array}{l}\text { B1dg } \\
\text { Range }\end{array}$ & $\begin{array}{l}0.289 \\
0.187\end{array}$ & $\begin{array}{l}0.344 \\
0.188\end{array}$ & $\begin{array}{l}0.151 \\
0.00\end{array}$ & $\begin{array}{l}0.151 \\
0.00\end{array}$ & $\begin{array}{l}0.147 \\
0.107\end{array}$ & $\begin{array}{l}0.161 \\
0.107\end{array}$ & $\begin{array}{r}0.071 \\
-0.101\end{array}$ & $\begin{array}{l}0.071 \\
0.101\end{array}$ \\
\hline
\end{tabular}


The values for these correlations are presented in Table VIII.

None of the values is significant.

The data do not support the hypothesis, although the direction of the nonsignificant correlations is in the one predicted.

The second hypothesis dealing with the strategy of filtering potentia1 interaction states:

3b) Children living in housing environments with a greater number of peers interact with a smaller proportion of the potential playmates in the environment than children living in housing environments with fewer peers.

Measures of both the interaction potential (specified by the hypothesis) and measures of the contact-potential will be compared. There are four sets of measures of the dependent variable. The first set of measures, the percent of peers in the play-group, is the measure specified by the hypothesis. However, as was explained earlier, it cannot be taken as given that a distinction between contact and interaction is necessary for studying children's interaction. As a result, the measure "percent of people in play-group," will also be used. Further, designing the measures as a ratio pernits an absolute comparison of the measures, since they are all reported in equivalent units (percentages). However, perhaps there is some absolute number of people that is significant for interaction; perhaps interaction is scaled not in terms of some ratio available contacts but rather in terms of some absolute optimal number. Thus, two more measures are added: number of peers and number of people in the play-group. All four of these sets are measured in each of three situations: the day-care center, the home, and inside the dwelling. The values are reported in Table IX through Table XII. 
TABLE VIII

CORRELATIONS: DURATION AND

COMMUNICATIONS CHARACTERISTICS

$$
\text { Duration }
$$

\begin{tabular}{|c|c|c|c|}
\hline \multirow{2}{*}{ \#Peers: } & School & Home & Inside \\
\hline & & & -0.208 \\
\hline Range & -01250 & -0.173 & -0.262 \\
\hline $\begin{array}{l}\text { Bldg } \\
\text { Range }\end{array}$ & $\begin{array}{r}0.019 \\
-0.234\end{array}$ & $\begin{array}{l}-0.311 \\
-0.192\end{array}$ & $\begin{array}{l}-0.176 \\
-0.159\end{array}$ \\
\hline
\end{tabular}


The data do not support the hypothesis, as it is stated. Most of the correlations fail to achieve significant levels. Those that do achieve significant levels, and even the larger nonsignificant correlations, are in the opposite direction to that predicted by the hypothesis: To the extent that there is any relationship, it is that children in environments with high communication-potentials engage in more interaction. This is in keeping with the finding that cognitive structuring is occurring as an adaptive strategy--the children are not only tolerant of greater densities, they also play with greater numbers of people. The very high correlations with the number of people in the inside play-group when related to the number of peers in the child's range (but the very low correlations with percent of people in the inside play-group) suggests that in the areas where there are a lot of children they play inside, apparently under the parent's watchful eye (since there is not a high correlation with the number of peers in the play-group). Also note that the communication characteristics of the building have a relation with measures in the day-care situation as well as in the home situation.

Thus, there is no support for the hypothesis that high potential interaction is filtered by decreasing the number or proportion of people interacted with. There is weak and sketchy evidence that high interaction potential results in adaptation by the assimilation of that higher level of interaction into behavior patterns.

The third hypothesis dealing with the strategy of filtering potential interaction states:

3c) Children living in housing environments with a greater number of peers use fewer settings than children living in housing environments with fewer peers. 
TABLE IX

CORRELATIONS: \%PEERS IN PLAY-GROUP
AND COMMUNICATION CHARACTERISTICS

\%Peers

\begin{tabular}{l|l|l|l|}
\multicolumn{1}{c}{ \#Peers: } & \multicolumn{1}{c}{ School } & \multicolumn{1}{c}{ Home } & \multicolumn{1}{c}{ Inside } \\
\cline { 2 - 4 } $\begin{array}{c}\text { Bldg } \\
\text { Range }\end{array}$ & 0.237 & -0.062 & 0.266 \\
\#People & 0.143 & -0.035 & 0.186 \\
\cline { 2 - 4 } Bldg & 0.294 & 0.00 & 0.259 \\
Range & 0.138 & 0.031 & 0.265 \\
\hline
\end{tabular}

TABLE X

CORRELATIONS: 非PEERS IN PLAY-GROUP AND COMMUNICATION CHARACTERISTICS

\begin{tabular}{|c|c|c|c|}
\hline \multirow{2}{*}{ ת } & \multicolumn{3}{|c|}{ gpeodle } \\
\hline & School & Home & Inside \\
\hline $\begin{array}{l}\text { BIdg } \\
\text { Range }\end{array}$ & $\begin{array}{l}0.335 \\
0.153\end{array}$ & $\begin{array}{c}-0.065 \\
0.00\end{array}$ & $\begin{array}{l}0.232 \\
0.101\end{array}$ \\
\hline $\begin{array}{l}\text { Bldg } \\
\text { Range }\end{array}$ & $\begin{array}{l}0.376 * \\
0.243\end{array}$ & $\begin{array}{l}0.028 \\
0.035\end{array}$ & $\begin{array}{l}0.234 \\
0.206\end{array}$ \\
\hline
\end{tabular}

* Sig. at .05 level 
TABLE XI

CORRELATIONS: \#PEERS IN PLAY-GROUP

AND COMMUNICATION CHARACTERISTICS

\begin{tabular}{l|l|l|l|}
\multicolumn{3}{c}{ \#Peers } \\
\multirow{2}{*}{ \#Peers: } & School & Home & Inside \\
\cline { 2 - 5 } Bldg & 0.216 & -0.133 & 0.176 \\
Range & 0.112 & -0.055 & 0.197 \\
\cline { 2 - 4 } \#People: & & & \\
Bldg & 0.227 & -0.124 & 0.094 \\
Range & 0.129 & -0.061 & 0.159 \\
\hline
\end{tabular}

TABLE XII

CORRELATIONS : \#PEOPLE IN PLAY-GROUP AND COMMUNICATION CHARACTERISTICS

\begin{tabular}{|c|c|c|c|}
\hline \multirow{2}{*}{ \#Peers: } & \multicolumn{3}{|c|}{ \#People } \\
\hline & School & Home & Inside \\
\hline $\begin{array}{l}\text { B1dgeers: } \\
\text { Bange }\end{array}$ & $\begin{array}{l}0.294 \\
0.059\end{array}$ & $\begin{array}{l}0.00 \\
0.00\end{array}$ & $\begin{array}{l}-0.039 \\
0.903 *\end{array}$ \\
\hline $\begin{array}{l}\text { ifreople: } \\
\text { Bldg } \\
\text { Range }\end{array}$ & $\begin{array}{l}0.294 \\
0.180\end{array}$ & $\begin{array}{l}0.055 \\
0.00\end{array}$ & $\begin{array}{l}0.049 \\
0.843 *\end{array}$ \\
\hline
\end{tabular}


Measures of both the interaction-potential (specified by the hypothesis) and measures of the contact-potential will be compared. The dependent variable is measured by the average number of settings used by the child in each of three situations: the day-care center, the home, and inside the dwelling, and it is also measured by the area of the child's range. The values for these correlations are presented in Table XIII.

The hypothesis is not supported by the data. Although the direction of some of the correlations is that predicted by the hypothesis, the magnitudes are so low as to discourage any speculation. The only strong correlations are between the number of peers in the child's range and the size of the range: the larger the range, the more children in it. This finding is not as trivial as might appear at first. It was not known whether the range would be used to filter interaction. If it were to serve that purpose, then there would be no correlation between the number of peers and size of range: all children would maintain an optimal level of social stimulation, expanding or contracting their range to maintain that optimal level. It will be recalled that this is Calhoun's thesis, ${ }^{2}$ among others. The evidence in this study does not support the thesis, but suggests instead that range is not a function of the number of social interactions it contains.

To summarize briefly before going on to consider other findings: It has been found that the first and second hypotheses are supporte? by the results of this study. It appears that cognitive structuring and some withdrawal from interaction are used as strategies for adapting to the communication-characteristics of the housing environment. There was little evidence that filtering of potential interactions was used an an adaptive strategy. The possibility remains, however, that different 
TABLE XIII

\section{CORRELATIONS : 非SETTINGS AND COMMUNICATION CHARACTERISTICS}

\begin{tabular}{|c|c|c|c|c|}
\hline & \multicolumn{4}{|c|}{ Settings } \\
\hline & School & Home & Inside & Range \\
\hline $\begin{array}{l}\text { \#Peers: } \\
\text { Bldg } \\
\text { Range }\end{array}$ & $\begin{array}{r}0.194 \\
-0.150\end{array}$ & $\begin{array}{l}-0.149 \\
-0.099\end{array}$ & $\begin{array}{l}-0.167 \\
-0.198\end{array}$ & $\begin{array}{r}-0.260 \\
0.786^{*}\end{array}$ \\
\hline $\begin{array}{l}\text { \#People: } \\
\text { Bldg } \\
\text { Range }\end{array}$ & $\begin{array}{l}0.113 \\
0.00\end{array}$ & $\begin{array}{l}-0.031 \\
-0.053\end{array}$ & $\begin{array}{l}-0.145 \\
-0.173\end{array}$ & $\begin{array}{r}-0.195 \\
0.582 \star\end{array}$ \\
\hline
\end{tabular}


groups may use different strategies. Such a possibility cannot be ruled out by this study.

\section{OTHER FINDINGS}

An analysis of some of the other measures collected in this study throws additional light on the relationship between the housing environment and children's behavior.

In considering the strategy of cognitive structuring, it was pointed out that it was not clear whether the relationship was due to the socio-spatial dimension or the physical dimension of the housing environment, since the two were so highly correlated. The correlation between Desor's test and the number of units in the building (a key measure of the physical dimension) was 0.621 (Table XIV). This correlation is, however, smaller than the correlation of the number of peers in the building with Desor's test, which was 0.634 . The difference is very small and could easily be due to random variance in the measures. But it does suggest that, if the difference holds constant in retests, the effect is due to the socio-spatial dimension of the housing environment.

The analysis of cognitive structuring can be extended by considering the interrelated effects of other measures that are also correlated with a child's score on Desor's test. The only other significant correlations are with Age of Child and Size of Unit (Table XIV). Returning to the matrix of correlations for the independent variables (Table II), it can be seen that the correlation between the Size of the Unit and the Number of peers in the building was -0.37 . While no correlation coefficient was reported for Age of Child and the Number of peers in the building, the t-Test for differences of means for the dwelling-type dichotomy 
TABLE XIV

CORRELATIONS: COGNITIVE STRUCTURING AND CONTROL VARIABLES

$$
\begin{aligned}
& \text { 非位s Age Size of Unit } \\
& \begin{array}{ll|l|l|l|}
\hline \text { Desor's Test } 0.621 * & -0.355^{*} & -0.383^{*} \\
\hline
\end{array} \\
& \text { * Sig. at .05 level }
\end{aligned}
$$

TABLE XV

REGRESSION ANALYSIS:

COGNITIVE STRUCTURING

Desor's Test
\#Peers $0.634^{*}$
Age $0.703^{*}$
Size $0.712 *$
* Sig. at .05 1evel


(Table III) was not significant, suggesting that the correlation between Age and Number of peers would be small, if any. These three correlates of cognitive structuring can be interrelated through a multiple regression. It would be expected that Number of Peers, correlated most highly with Desor's test, would be entered first in a stepwise regression, and that Age, which is probably the less correlated with Number of peers, would be entered second, with the Size of unit being entered last. Table XV shows that this expected result was obtained. The three measures together account for half the variance (a Multiple $\underline{R}$ Square of 0.506 ) in cognitive structuring; with the Number of peers alone accounting for fourfifths (a Multiple $\underline{R}$ Square of 0.402 ) of the relationship.

There was some concern at the outset that exposure to the situations could be a confounding variable in the study. The confounding effect could operate through either of two channels. Following Sonnenfeld's suggestion that'natives differ from nonnatives, ${ }^{3}$ possibly the simple fact of having moved could influence the child's perception. Recall that it was found in Table III that multi-family dwellers moved more recently than single-family dwellers. Second, the confounding effect could also operate through awareness of one's environment: perhaps children who have woved recently have met fewer friends or have found fewer places to play. A parallel set of possibilities could be posited for the number of months the child has been going to the day-care center: perhaps the newer children have fewer friends and have found fewer places to play at the center. The child's exposure to his current situation was defined in three ways: by the percent of his life that has been spent in the current type of building (thus controlling for differences in age), by the number of months he has spent in the culrent type of building, and by the number of 
months he has lived in his current building. This last measure is more crucial for the second mode of confounding an exposure effect, since it focuses on familiarity with the characteristics of the specific situation, rather than adaptation to the general type of situation. The result of these three measures are reported in Table XVI through XVIII. The child's exposure to the day-care center was defined only by the number of months at the day-care center: Since all the children were in the day-care situation currently, and since the situation is not a dwelling environment, the native/nonnative distinction did not seem to apply. Those results are reported in Table XIX.

The only significant values in any of the tables are in the "Duration" row. In the first three tables (concerned with exposure to the housing environment), the significant correlations are with duration of behavior episodes in the housing environment. In the fourth table (concerned with exposure to the day-care environment), the significant correlation is with the duration of behavior episodes in the day-care environment. Note that the highest values for exposure to the housing environment occur with the number of months in the current building.

In other words, there is strong evidence that there is a moderate relation between duration of activity and familiarity with the environment in which it occurs. It will be recalled that there were no significant differences between multi-family and single-family dwellers regarding esposure to the day-care center; but there was a significant difference in exposure to the current housing environment (multi-family dwellers having had less exposure) (Table III). It will also be recalled that no significant relationship was found for the duration of activity when related to the communication-structure of the housing environment (Table VIII). 
TABLE XVI

CORRELATIONS: DEPENDENT MEASURES

AND \% LIFE IN BLDG-TYPE

\begin{tabular}{|c|c|c|c|c|}
\hline $\begin{array}{l}\text { Dependent } \\
\text { Measures I }\end{array}$ & Interview & School & Home & Inside \\
\hline Desor & -0.133 & & & \\
\hline P-Solitary & 0.023 & & & \\
\hline$\%$ Solitary & & 0.064 & 0.125 & 0.137 \\
\hline Refuse & & 0.099 & 0.232 & 0.170 \\
\hline Ignore & & 0.038 & 0.246 & 0.170 \\
\hline Duration & & 0.070 & $0.418 *$ & 0.066 \\
\hline Range & -0.079 & & & \\
\hline Settings & & 0.214 & $-0: 173$ & -0.278 \\
\hline$\%$ Peers & & -0.049 & 0.115 & -0.208 \\
\hline \# Peers & & -0.099 & $-0: 165$ & -0.058 \\
\hline$\%$ People & & 0.129 & 0.038 & -0.276 \\
\hline \# People & & 0.110 & 0.037 & 0.139 \\
\hline
\end{tabular}


TABLE XVII

CORRELATIONS: DEPENDENT MEASURES

TIME IN BLDG-TYPE

Dependent

Measures

Interview School Home Inside

Desor

$-0.169$

\begin{tabular}{ll} 
P-Solitary & -0.081 \\
\hline
\end{tabular}

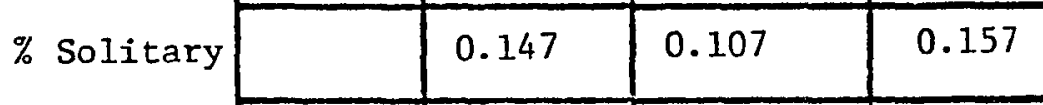

Refuse

Ignore

Duration

Range

Settings

$\%$ Peers

\# Peers

\% People

\# People

\begin{tabular}{|l|l|l|l|}
\hline & 0.134 & 0.279 & 0.159 \\
\hline & 0.093 & 0.233 & 0.159 \\
\hline & 0.165 & $0.369 *$ & 0.279 \\
\hline & & & \\
\hline & 0.148 & -0.089 & -0.293 \\
\hline & 0.020 & 0.114 & -0.213 \\
\hline & -0.089 & 0.102 & -0.156 \\
\hline & 0.183 & 0.097 & -0.254 \\
\hline & 0.129 & 0.00 & 0.111 \\
\hline
\end{tabular}

* Sig. at .05 level 
TABLE XVIII

CORRELATIONS: DEPENDENT MEASURES

AND TIME IN BLDG

\begin{tabular}{|c|c|c|c|c|}
\hline $\begin{array}{l}\text { Dependent } \\
\text { Measures }\end{array}$ & Interview & School & Home & Inside \\
\hline Desor & -0.241 & & & \\
\hline P-Solitary & 0.051 & & & \\
\hline$\%$ Solitary & & -0.142 & -0.090 & -0.132 \\
\hline Refuse & & -0.080 & 0.273 & 0.00 \\
\hline Ignore & & 0.202 & -0.091 & 0.249 \\
\hline Duration & & 0.111 & $0.519 *$ & $0.455^{*}$ \\
\hline Range & 0.336 & & & \\
\hline Settings & & 0.00 & -0.205 & -0.154 \\
\hline$\%$ Peers & & -0.073 & 0.158 & -0.062 \\
\hline \# Peers & & -0.234 & 0.166 & 0.058 \\
\hline$\%$ People & & 0.193 & -0.084 & -0.348 \\
\hline \# People & & -0.019 & 0.00 & -0.030 \\
\hline
\end{tabular}


TABLE XIX

CORRELATIONS: DEPENDENT MEASURES

AND TIME AT HGC

\begin{tabular}{|c|c|c|c|c|}
\hline $\begin{array}{l}\text { Dependent } \\
\text { Measures }\end{array}$ & Interview & School & Home & Inside \\
\hline Desor & -0.050 & & & \\
\hline P-Solitary & -0.175 & & & \\
\hline$\%$ Solitary & & 0.038 & 0.256 & -0.082 \\
\hline Refuse & & -0.101 & 0.221 & 0.341 \\
\hline Ignore & & -0.082 & 0.251 & 0.222 \\
\hline Duration & & $-0.356 *$ & -0.174 & -0.279 \\
\hline Range & 0.243 & & & \\
\hline Settings & & -0.048 & 0.125 & 0.140 \\
\hline$\%$ Peers & , & 0.038 & -0.269 & 0.00 \\
\hline \# Peers & & 0.125 & -0.209 & 0.107 \\
\hline \% People & & -0.204 & -0.154 & 0.042 \\
\hline \# People & & 0.00 & -0.187 & 0.347 \\
\hline
\end{tabular}


although a tendency was found in the data that suggested support. This finding of a relationship due to exposure suggests that, while there may be no significant relationship due solely to the communication-characteristics of the environment, when exposure to that environment is considered, such a relationship may be found. In other words, single-family and multifamily children may still differ in the duration of their behavior episodes, although the relationship is due only in part to the socio-spatial dimension of the housing environment. A set of $t$-Tests were computed (Table $\mathrm{XX}$ ). No significant differences were found between single-family and multi-family childrer for duration of activity in either the day-care or inside the dwelling situations. A significant difference was found for duration of activity in the home situation. These results can be considered to give limited support to the hypothesis that the duration of activity is related to (a) exposure (b) to the communication-characteristics of the housing environment.

There has also been some suggestion in the literature that certain types of interactions are related to children's sex roles. 4 They are not supported in this study (Table XXI). A set of t-Tests to test the difference in children's behavior by sex revealed no significant differences. These results should not be taken as totally invalidating such studies, however. The University community is atypical of the larger American culture, and it is likely that the traditional sex-roles are purposely and determinedly not transmitted to the children at the University's day-care center.

Finally, some effort was made to determine whether the children themselves were aware of single-family/multi-family differences among themselves. The children's choice of playmates at the day-care center 
TABLE XX

t-TEST: DURATION OF EPISODES FOR SINGLE-FAMILY AND MULTI-FAMILTY CHILDREN

\begin{tabular}{|c|c|c|c|}
\hline & School & Home & Inside \\
\hline Duration & 1.115 & $2.509 *$ & 0.743 \\
\hline
\end{tabular}

* Sig. at .05 leve1

TABLE XXI

t-TEST: DIFFERENCES IN DEPENDENT

MEASURES BETWEEN BOYS AND GIRLS

\begin{tabular}{|c|c|c|c|c|}
\hline $\begin{array}{l}\text { Dependent } \\
\text { Measures }\end{array}$ & Interview & School & Home & Inside \\
\hline Desor & 0.238 & & & \\
\hline P-Solitary & 0.111 & & & \\
\hline$\%$ Solitary & & 1.207 & 0.885 & 0.972 \\
\hline Refuse & & 0.221 & 0.745 & 0.907 \\
\hline Ignore & & 0.565 & 0.591 & 0.688 \\
\hline Duration & & 0.482 & 0.578 & 0.516 \\
\hline Range & 1.189 & & & \\
\hline Settings & & 0.578 & 0.305 & 1.451 \\
\hline$\%$ Peers & & 0.388 & 0.728 & 0.695 \\
\hline \# Peers & & 0.359 & 0.691 & 1.028 \\
\hline \% Prople & & 0.472 & 0.110 & 1.039 \\
\hline \# People & & 0.691 & 0.594 & 1.131 \\
\hline
\end{tabular}


was examined to see whether the children chose their friends from among their own group. The first chi-square analysis (Table XXII) examined the total number of interactions, rejecting no choices. No significant differences were found. However, when the choice of reighbors (other children living within the child's range) were excluded (Table XXIII), it was found that multi-family children play with other multi-f"mily children significantly more than they play with children from single-family dwellings, unless the single-family children happen to be neighbors. In other words, it appears that the children are aware of the differences in each other, differences which are related to the housing environment.

\section{DISCUSSION}

The results of the analysis of the hypotheses will now be turned to a discussion of the broader issues raised earlier.

In the third chapter, a conceptual distinction was made between the physical and socio-spatial dimensions of the housing environment, based on the work of Terrence Lee and Chalsa Loo.5 The usefulness of such a distinction in this study can now be evaluated. It was found in Chapter $V$ (Table II) that one set of measures which pruportedly measured the socio-spatial dimension of the housing environment was highly correlated to measures of the physical dimension, both in the magnitude of the correlation coefficients and in the similarity of patterns of correlations with measures of the social dimension. A second set of socio-spatial measures was not part of this pattern. In the analysis of the first hypothesis, dealing with the strategy of cognitive structuring, it was found that the measure of cognitive structuring was correlated with the measure of the socio-spatial dimension of the housing environment which was highly correlated with measures of the physical dimension (Table IV). In the anal- 
TABLE XXII

CHI-SQUARE: INTERACTION

CHOICES. ALL CHOICES

\begin{tabular}{|c|c|c|}
\hline & $\begin{array}{l}\text { Multi- } \\
\text { Family }\end{array}$ & $\begin{array}{l}\text { Single-- } \\
\text { Family }\end{array}$ \\
\hline $\begin{array}{l}\text { Multi - } \\
\text { Family }\end{array}$ & 74 & 48 \\
\hline $\begin{array}{l}\text { Single- } \\
\text { Family }\end{array}$ & 62 & 50 \\
\hline & & \\
\hline
\end{tabular}

Chi-square $=1.7980$ $\mathrm{df}=1$

Not Significant at .05 level

TABLE XXIII

CHI-SQUARE : INTERACTION

CHOICES. MINUS NBRS

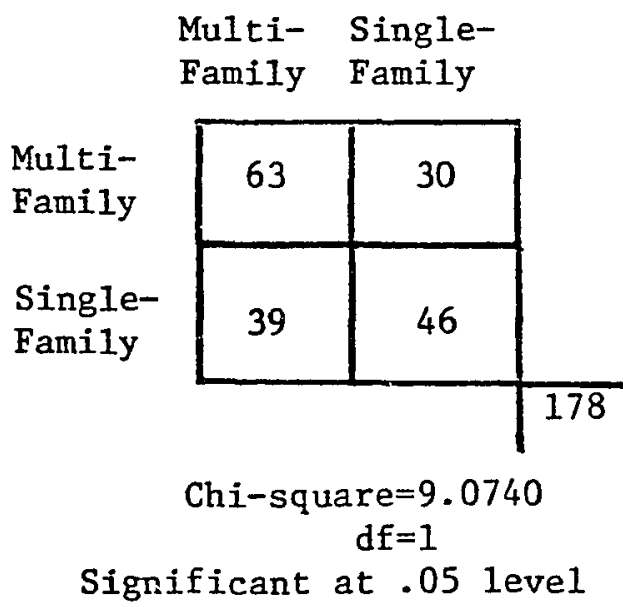


ysis of the second hypothesis, dealing with rejection of interaction, a clear effect due to socio-spatial measures was found, since the most powerful measures were from the set of measures that was not highly correlated with the measures of the physical dimension (Table V and Table VI). However, a t-Test failed to find a significant difference between singlefamily and multi-family dwellings on those measures of the socio-spatial dimension (Table III). In other words, where there is clear evidence of the significance of a distinction between the physical and socio-spatial dimensions of the housing environment, the usefulness of the measures for the purposes of this study is unclear, since those measures are not distinguishable in the two classes of housing environments which were considered. Where the measures are clearly distinguishable (e.g., characteristics of the building population) by dwelling-type, their high covariance with measures of the physical dimension makes discr Jery of clearly significant differences unlikely, although the data does tend to support the possibility that there are real differences.

In the same article mentioned above, Lee concluded on the basis of his research that an individual's range is a function of distance and is not influenced by the number of people within it. 6 Calhoun, on the other hand, suggested that an individual's territory is a function of the social interactions it contains, that one extends or contracts one's range in order to maximize reinforcing interactions. 7 While not originally designed to test between these two hypotheses, the results of this study happen to throw some light on it. It was found (Table IIII) that the size of a child's range is highly correlated with the number of people in the range, especially the number of children. In other words, the larger the range the more potential contacts and the more potential inter- 
actions it contains. This is in agreement with Lee's findings. It is not, however, a refutation of Calhoun's position. In the first place, it is not clear in what sense a child's range is comparable to an adult's: a child's range may be artificially restricted, both by parental commands and by environmental barriers that are not present for adults. Further, there is some support for Calhoun's position (or that part of Calhoun's position that is derived from Cristaller's theory) in Plattner's article, 8 which showed a macro-level structure that is similar to what Calhoun predicted. It should be pointed out that Plattner's model was based on economic and geographical variables, and Calhoun's is based on psychological variables. In any event, the final word has yet to be said in regard to Calhoun's theory.

Another conceptual distinction that must be evaluated was the distinction between interaction-potential and contact-potential. The distinction was derived from the work of Harvey Cox and Lyn Lofland, 9 and it was suggested that a distinction might be made by children between peopleto-be-played-with (peers) and others (adults). This distinction was offered as only an hypothesis, which would be evaluated in the course of analyzing the hypotheses. The correlation matrix (Table II) shows that the measures of contact-potential and interaction-potential were highly correlated, suggesting that the distinction, as measured, would not be useful. In hypothesis 1, however, a small difference was found in the relation of the contact and interaction measures with a measure of cognitive structuring (Table IV). A similar situation was found in analyzing hypothesis 3 in regard to the duartion of activity (Table VIII).

These findings amount to nothing more than tendencies. However, the analysis of hypothesis 2 in regard to rejecting interaction found 
clear differences between measures of contact potential and measures of interaction potential ( $T a b l e ~ V$ and Table VI). It was found that the contact potential of the housing environment has a significant relation with ignoring interaction, and the interaction potential of the housing environment has a significant relation with rejecting interaction. This difference, however, may be due to cultural constraints: children in our culture are not generally permitted to tell adults to "go away." The findings, then, do support the distinction between contact and interaction. The support, however, is weak and suggests that the effective difference between the two classes of measures is minimal for most behaviors.

Finally, some consideration must be given to the question of whether the behaviors observed in this study are "functional unities." In analyzing the multitrait-multimethod matrix, it was found that there was little convergence between the measures (Table I). Measures of a 'trait' in one environment had little relation to similar measures of that 'trait' in a different environment. The exceptions to this general rule were the measures of play-group size, where a moderate relationship was found. The analysis of the hypotheses conforms to that sumary. Whenever a significant relationship was found between the housing environment and behavior, it was always with behavior occurring in that same environment. The only exception was when the size of the child's play-group was examined. It was found that the housing environment related significantly to the size of the play-group at the day-care center as well. It was also noted that the direction of the relationshlp (the greater the communication characteristics of the housing environment, the larger the play-group) does not support the hypothesis that a strategy of filtering interaction is employed, but rather supports the hypothesis that cognitive structuring has 
occurred.

This finding, that cognitive structuring occurs in children as an adaptation to the housing environment, if it is confirmed by further research, is the most significant finding of this study. It helps explain why some people like big cities and some people like sleepy little towns. It supports Sonnenfeld's distinction between natives and nonnatives, 10 since it suggests that cognitive schema really are structured by the environment. And it even supports Sommer's suggestion that children 'imprint' to the environment they were raised in:11 the early environment might provide the basic cognitive structure against which all later environmental experiences are evaluated. It provides a beginning-point from which to understand how people experience the city. 
1. Simone de Beauvoir, The Ethics of Ambiguity. B. Frechtman, tr., New York: Citadel, 1948, p. 130 .

2. John B. Calhoun, "Space and the strategy of 1ife," in A. Esser; ed., Behavior and Environment, New York: Plenum, 1971.

3. Joseph Sonnenfeld, "Variable values in space and 1andscape: an inquiry into the nature of environmental necessity," Journal of Social Issues, 1966, 22(4), 71-82.

4. Brian C. Bates, Effects of Social Density on the Behavior of Nursery School Children, unpublished Ph.D. dissertation, University of Oregon, 1970; Martha C. Gustin, "Relationhsip between play area and cognitive style in pre-school children," Child Study Journa1, 1974, 3, 51-67.

5. Terrence Lee, "Urban neighborhoods as a socio-spatial schema," Human Relations, 1968, 21, 241-267; Chalsa Loo, "Important issues in-researching the effects of crowding on humans," Representative Research in Social Psychology, 1973, 4, 219-226.

6. Lee, op. cit.

7. Calhoun, op. cit.

8. Stuart Plattner, "Rural market networks," Scientific American, $1975, \underline{232}(5), 66-79$.

9. Harvey Cox, The Secular City, New York: Macmillan, 1966;

Lyn Lofland, A World of Strangers, New York: Basic Books, 1973.

10. Sonnenfeld, op. cit.

11. Robert Sommer, "Man's proximate environment," Journal of Social Issues, $1966,22(4), 59-70$. 
CHAPTER VII

SURIARY AND SUGGESTIONS FOR ADDITIONAL RESEARCH

Out of all things there comes a unity, and out of a unity all things. 1

\section{SURMARY OF FIINDINGS}

Three hypotheses, some with multiple statements, were tested in this study.

The first hypothesis dealt with the strategy of cognitive structuring in adaptation to the housing environment. It was found that children living in buildings with more peers show a greater acceptance of social density than children living in buildings with fewer peers. This relationship was found for the socio-spatial measures of the building environment, but not for the socio-spatial measures of the child's neigh- . borhood. It was further found that this strategy of cognitive structuring expressed itself in behavior. Children living in buildings with more peers also played with more people both inside the home and at the daycare center. This cross-situational relationship suggests that the strategy of cognitive structuring is a "functional unity," or a consistent pattern of the child's personality.

The second set of hypotheses dealt with the strategy of withdrawal from interaction in adaptation to the housing environment. The first statement of the general hypothesis dealt with rejection of interaction. It was found that children Iiving in neighborhoods with more peers were 
more likely to refuse overtures of interaction, and children living in neighborhoods with more people were more likely to ignore interaction than children living in neighborhoods with lower communication-potentials. It was noted that while this is a relationship with the housing environment, it will not distinguish between children in single-family and multi-family dwellings, since there were no significant differences between the two groups in the population characteristics of their neighborhoods. The second statement of the general hypothesis dealt with the amount of solitary play the children engaged in. No significant differences were found.

The third set of hypotheses dealt with the strategy of filtering potential interaction in adaptation to the housing environment. The first statement of the general hypothesis dealt with the allocation of time. No simple relationship was found between the communication characteristics of the housing environment and duration of the children's activity, although there was a tendency in support of the hypothesis that the greater the number of people in the building, the less time the children spent at any activity. Since it was noted that children from multi-family dwellings, on the average, had lived in their current dwelling a shorter time than children from single-family dwellings, it seemed likely that an interactive effert might be found. When the hypothesis was examined, then, in terms of dwelling-type, a significant relationship was found. The second statement of the general hypothesis dealt with the size of the play-g,roup. No support was found for the hypothesis that high interaction-potential is filtered by decreasing the number or proportion of people interacted with. In fact, evidence to the contrary was found. The third statement of the 
general hypothesis dealt witn the use of settings. No support was found for the hypothesis that high interaction-potential is filtered by use of fewer settings.

In summary, the first hypothesis was strongly supported, and it appears that the relationship is cross-situational. There was some support for some facets of the other two hypotheses, and the relationship appears to be due to the impact of the immediate environment. That only some of the findings were significant was not surprising: the intention, after al1, was to evaluate Milgram's theory, ${ }^{2}$ and to attempt to determine which of the several strategies he listed would be most fruitful for further research. That information was gained by this study.

Finally, it should be noted that the findings in this study are based on correlations. They indicate a relationship between two variables. A relationship between two variables is not the same as an effect of one upon the other. While it is unlikely that the child's behavior had an effect on the choice of housing environment (other, perhaps, than the mere fact of his being born--there is, after all, that conviction that apartments are nasty places to raise children), there is a third possibility other than the one that the housing environment had an effect on the crild's behavior. There may be some third variable, not measured in this study, to which both of these variables are related and which is responsible for their covariance. With this caveat in mind, we turn to suggestions for additional research.

\section{SUGGESTIONS FOR ADDITIONAL RESEARCH}

From the outset, the necessity for replication of this study has 
been rocognized. Relatively homogeneous groups from other social environments--for example, working class children, children from larger cities with larger apartment districts than Portland has, children who do not attend a day-care center--would be used as the subjects in such replications. In the course of this study, other dimensions of future replications were also uncovered.

Some refinement of the measures is now possible. Because the measures of the independent variables cluster together so strongly, it might be useful to derelop an index of measures in each cluster. While the development of an index of dwelling-types (rather than a simple dichotomy) would make the analysis more sensitive than a single dichotomy, the key variable in each cluster should probably still be examined individually as well. The single-family/multi-family dichotomy makes intuitive sense, it will be the schema people will use to understand the findings, and it will be useful to be able to know to what extent the intuitive relationship is modified when more dimensions are taken into account.

The findings of this study suggest that there were really two sets of measures of the dependent variables. The first set, composed of Desor's test and the measures of play-group size, were measures of what might be a personality triat which has trans-situational relationship with the home environment. The second set, composed of the other behavioral measures such as refusing overtures for interaction or duration of activity, were measures of pressure on behavior due to the immediate environment. Much more work will be needed to plumb the depths of either of these sets of measures. Future studies might do well to concentrate on specifying one or the other of these sets and be content with rudimentary measures of the 
other set (for purposes of control or for examining interactive effects.) It would also be worthwhile to examine some of the variables which were uncovered in the course of the study. An index of the depth of interaction might well be developed. It could be constructed not only from the measures used in this study, but also from a measure of the number of enclosed episodes. The provision of play-space in the home environment and the degree of acoustical privacy might also be profitably studied.

Greater sophistication in technique should also be introduced in the replications. It is not clear what effect the scale of the episode has on the behavior found in the field observation: are the differences between multi-family and single-family children such that only a very fine-grained resolution can discover them? If this is so, what does it mean? Is a very fine-grained effect also a less signigicant effect? This is not the place to attempt to answer that last question, but I suspect the answer will be similar to the answer to the question, "What are the effects of the intangibles in an economic analysis?"

Some effort should also be expended to enlarge our understanding of Desor's test. The validity of the test (other than its face validity) has never been considered. How well are subjects able to actually "put themselves" in the room represented by the apparatus? Some of the children put more than thirty figures in a space representing a room 9x18 feet. Would they feel "not crowded" if they were actually in such a situation? We also have no estimates of test-retest reliability. How stable are the scores for Desor's test? If they are unstable, what is the standard error, and how is it to be explained, what are its correlates? Third, there is only a little research on the effect of different configurations on 
performance. What are the psychological equivalents in dimensions:

Desor discussed the relationship between square and rectangular dimensions, but the limits have not been tested. Nor do we know how much of a change in size is necessary to get twice as many (or half as many) fig- • ures in a room. Finally, no developmental studies have been done. We do not know if the relevant dimensions of the apparatus for adults are also relevant for children, or hri the child's response changes with mental development. Desor's test was found to be a useful measure in this study. Were it better understood, it could become a very powerful tool in the arsenal of the environmental psychologist and the urban theorist.

Another refinement of technique would be to establish the reliability of all the measures. This would involve two steps, establishing the reliability of the observer and establishing the stability of the behavior observed. In this study, the reliability of the coding was estab1ished by comparing the Investigator's coding to the coding of an independent coder. Similarly, the reliability of the observation could be established by comparing the protocols of the Investigator to the protocols of an independent observer. This procedure could be problematic in a field setting, since the ideal would be for the independent observer to spot-check from behind a one-way mirror, or at least to spot-check from time to time. But this would certainly have been a disruptive procedure in the day-care center, and probably would be disruptive in any but the more public settings. In the early period of observation, when the Observer is establishing his presence and his routine rather than collecting protocols for later analysis, a pretest for inter-observer reliability could be performed: since the routine is already disrupted by the pre- 
sence of one stranger, perhaps the presence of a second stranger would result in only minimal additional disruption. The second step in establishing reliability is to test for the stability of the measures. In this study, the use of the mean value for several observations as the test measure minimized much of the variability.

The standard deviation of the test measures could be used as one estimate of reliability. However, a wide variance may be a stable characteristic of some measures. The best estimate would be derived from either a test-retest or split-halves measure of reliability. Either of these techinques would require a large number of observations, larger than the number used in this study. Consequently, estimates of the stability of the measures would probably not be attempted until toward the end of a program of research when precisely the right measures are being used, because the gathering of enough observations will be a time-consuming and expensive process.

Besides these sophistications in technique, there are several tactical recomendations for future research. It would be useful, now, to replicate the study with children who are not attending a day-care center. Besides the problem of obtaining subjects, discussed earlier, there would also be the problem of finding some sort of common, or at least comparable, non-home-environment setting in which to observe the children. The comparison between the HOME and SCHOOL environments permitted an analysis of whether a relationship was due to environmental pressure or was trans-situational, and the advantage of that comparison should not be given up. The advantage to studying non-day-care children will lie in the potential for comparison with the subjects of this study. Very few 
differences were found in style of interaction (e.g., use of settings, solitary play), but it cannot be said whether this failure was because there is no relationship, or because the day-care experience has effaced it. When such a replication is performed, it would probably prove advantageous to develop an index for "maximizing interaction," composed of the number of settings used, the duration of play, and the size of the playgroup. It would also be useful to consider a measure of the ratio of enclosing to enclosed episodes.

It would also be very useful if a greater separation between the measures of the physical and socio-spatial dimensions of the building environment could be obtained. This would require children living in buildings roughly the same in the number of units with different numbers of people and children (or, vice-versa, different numbers of units with the same population size). Large apartments are hard to find: two or three bedrooms seem to be the largest. This does not permit much flexibility in family size; yet, assuming equal vacancy rates, it is only through family. size that a greater separation of the dimensions is possible. It is a puzzling problem.

Not at all puzzling is the need to study the effect of the housing environment on children's behavior in a developmental fashion. Having found a relationship in fairly young children, it sti11 remains to be seen whether and how this relationship is carried forward in the process of development. Cross-sectional studies of development might serve to follow the strength of the relationship found here. But a longitudinal, or at least an accelerated longitudinal, ${ }^{3}$ approach will be necessary to follow the transformation of the reilationship with developmental changes, 
whether cognitive, social, or emotional.

Firally, there is the problem of sample-size. The restriction of sample-size due to the need for a moderately homogeneous group would probably be alleviated somewhat by gathering the data in cities with larger apartment-dwelling populations. However, given the conviction that apartments are nasty places to raise children, the restriction on sample-size will probably not be avoided even there. The one alternative is to devise measures which can be administered to groups, or at least measures taken form a standard apparatus which can be administered by many assistants. Desor's test, of course, is just such a measure. One reason for the interview of parents was to see if measures obtained in that fashion would be correlated enough to the measures obtained from observation, so that the former could reasonably be used as a surrogate for the latter. The results were not encouraging. In any event, if standardized, groupadministered measures can be devised, school systems could be contacted and the entire school-age population of a city tested. With so large a sample, the non-systematic inter-group differences could be expected to have minimal impact on the study. Further, such a large sample would be ideal for cross-sectional developmental study.

This study began with the question, "How do people experience the city they live in, and how do the respond to that experience?" The question has not been answered, but at least we have a better idea of where to look for the answer. 
NOTES

1. Heraclitus of Ephesus, quoted in G.S. Kirk \& J.E. Raven, The Presocratic Philosophers, Cambridge: Cambridge University Press, $\overline{1971}$, p. 191.

2. Stanley Milgram, "The experience of living in cities," Science, $1970,167,1461-1468$.

3. R.Q. Bel1, "An experimental test of the accelerated longitudinal approach," Child Development, 1954, 25, 281-286. 
REFERENCES

Allen, Marjorie. Planning for Play. Boston: MIT Press, 1969.

Austin, Martha C. "Relationship between play area and cognitive style in pre-school children," Child Study Journal, 1974, 3, 51-67.

Barker, Roger G. "The Stream of Behavior as an Empirical Phenomenon," in R. Barker, ed., The Stream of Behavior. New York: Appleton, Century, Crofts, 1963.

Barker, Roger G. \& Herbert F. Wright. Midwest and Its Children. Hamden, Conn.: Archon, 1955.

Bates, Brian C. Effects of Social Density on the Behavior of Nursery School Children. unpublished Ph.D. dissertation, University of Oregon, 1970.

Bauer, Catherine. "Social questions in housing and community planning," Journal of Social Issues, 1951, I(1-2), 1-33.

Baum, Andrew \& Stuart Valins. "Architecture, social interaction, and crowding," Transactions of the New York Academy of Sciences, 1974, 36, 793-799.

Bechte1, Robert B. "A behavioral comparison of urban and small town environment," in J. Archea \& C. Eastman, eds., EDRA2. Pittsburgh, Pa., October, 1970.

Beck, Robert. "Spatial meaning and the properties of the environment," in D. Lowenthal, ed., Environmental Perception and Behavior. Chicago: University of Chicago, Department of Geography, Research Paper 109, 1967.

Becker, Franklin D. Design for living: the Residents' View of MultiFamily Housing. Ithaca, New York: Center for Urban Development, Cornel1 University, May, 1974.

Bell, R.Q. "An experimental test of the accelerated longitudinal approach," Child Development, 1954, 25, 281-286.

Bengtsson, Arvid. Environmental Planning for Children's Play. New York: Praeger, 1970 .

Blake, Robert, C.C. Rhead, B. Wedge, \& J.S. Mouton. "Housing architecture and social interaction," Sociometry, 1956, 19, 133-139. 
Blalock, Hubert M. Social Statistics, 2nd Ed. New York: McGraw-Hill, 1972.

Bossard, James H.S. "The law of family interaction," American Journal of Sociology, 1945, 50, 292-294.

Brolin, B. "Mass housing: Social research and design," Architectural Forum, 1968, 129, 66-71.

Brower, Sidney. "Streetfront and Sidewalk," Landscape Architecture, $1973,63(4), 364-369$.

Bruner, Jerome S. Beyond the Information Given. New York: Norton, 1973.

Burgess, Ernest W. "The growth of the city: an introduction to a research project," in R.E. Park, E.W. Burgess, \& R.D. Mckenzie, eds. The City. Chicago: University of Chicago Press, 1925.

Calhoun, John B. "The role of space in animal sociology," Journal of Social Issues, $1966,22(4), 46-58$.

-_._- "Space and the strategy of life," in A. Esser, ed., Behavior and Environment. New York: Plenum, 1971.

Campbell, Donald T. \& Donald W. Fiske. "Convergent and discriminant validation by the multitrait-multimethod matrix," Psychological Bulletin, 1959, 56, 81-105.

Carnahan, Douglas, Walter Gove \& Oner R. Galle. "Urbanization, population density, and overcrowding: Trends in the quality of life in urban America," Social Forces, 1974, 53, 62-72.

Carrothers, Gerald A.P. "An historical review of the gravity and potential concepts of human interaction," Journal of the American Institute of Planners, 1956, 22, 94-102.

Carstairs, George M. "Overcrowding and human aggression," in H.D. Graham \& T.R. Curr, eds. Violence in America: Historical and Comparative Perspectives. Washington, D.C.: U.S. Government Printing Office, 1969.

Center for Population Research and Census. "1970 Census tract data for Clackamas, Multnomah, and Washington counties." Portland State University (mimeo, n.d.)

- "Population pyramids by census tract for Portland part of Portland SMSA." Portland State University (mimeo, n.d.)

Chapin, F. Stuart. "An experiment on the social effects of good housing," American Sociological Review, 1940, 5, 868-879.

"The psychology of housing," Social Forces, 1951, 30, 11-15. 
Choldin, Harvey M. "Population density and social relations." Paper presented at meeting of Population Association of America, Toronto, April 14, 1972.

Chombart de Lauwe, Paul. Famille et Habitation, Tome I. Paris: Editions du Centre National de la Recherche Scientifique, 1959.

Famille et Habitation, Tome II. Paris: Editions du Centre National de la Recherche Scientifique, 1960.

Cole, Michael, John Gay, Joseph A. Glick, Donald W. Sharp. The Cultural Context of Learning and Thinking. New York: Basic Books, 1971.

Cooper, Clare. "The house as symbol," Design and Environment, 1972, 11, 30-37.

Cox, Harvey. The Secular City. New York: Macmillan, 1966.

Dattner, Richard. Design for Play. New York: Van Nostrand, 1969.

Dean, John P. "Housing design and family values," Land Economics, 1953, 29, $128-141$.

Desor, Jeanette A. The Psychology of Crowding: An Experimental Investigation. unpublished Ph.D. Dissertation. Cornell University, 1969.

- "Toward a psychological theory of crowding," Journal of Personality and Social Psychology, 1972, 21, 79-83.

Dickman, Harold. "The perception of behavioral units," in R. Barker, ed., The Stream of Behavior. New York: Appleton, Century, Crofts, 1963.

Draper, N.R: \& H. Smith. Applied Regression Analysis. New York: Wiley, 1966.

Dreiser, Theodore. Sister Carrie. New York: Doubleday, 1900.

Dubos, Rene. "Environmental determinants of human life," in D. Glass, ed., Environmental Influences. New York: Russell Sage, 1968.

Esser, Aristide H. "A biosocial perspective on crowding," In J.F. Wohlwill \& D.H. Carson, eds., Environment and the Social Sciences. Washington, D.C.: American Psychological Association, 1972.

Faris, Robert E.L. \& H. Warren Dunham. Mental Disorders in Urban Areas. Chicago: University of Chicago Press, 1939.

Festinger, Leon, Stanley Schachter, \& Kurt W. Back. Social Pressure in Informal Groups. New York: Harper, 1950.

Fischer, Claude S. "Urbanism as a Way of Life': A review and an agenda," Sociological Methods and Research, 1972, I, 187-242. 
Forrester, Jay W. Urban Dynamics. Cambridge: MIT Press, 1970.

Franke, J. \& J. Bortz. "Contributions to the application of psychology In city planning. I." Zeitschrift fur experimentelle und Angewandte Psychologie, 1972, 19, 76-1-8.

Fraser, Thomas M. "Density, intensity, and stress," Ekistics, 1972, 33; 269.

Freedman, Jonathan, Simon Klevansky \& Paul Ehrlich. "The effect of crowding on human task performance," Journal of Applied Social Psychology, 1971, 1, 7-25.

Fried, Marc \& Peggy Gleicker. "Some sources of residential satisfaction in an urban slum," Journal of the American Institute of Planners, 1961,$27 ; 305-315$.

Friedberg, M. Paul, with Ellen Perry Berkeley. Play and Interplay. New York: Macmillan, 1970.

Galle, O.R., W.R. Gove, \& J.M. McPherson. "Population, density, and pathology: What are the relations for man?" Science, 1972, 176, 23-30.

Glaser, Barney G. \& Anselm L. Strauss. The Discovery of Grounded Theory. Chicago: Aldine, 1967.

Griffit, William \& Russell Veitch. "Hot and crowded: Influences of population density and temperature on interpersonal affective behavior," Journal of Personality and Social Psychology, 1971, 17, 92-98.

Grootenboer, E.A. "The relation of housing to behavior disorder," American Journal of Psychiatry, 1962, 119, 469-472.

Guilford, J.P. Fundamental Statistics in Psychology and Education, 4th ed. New York: McGraw-Hill, 1965.

Gutman, Robert. "Site planning and social behavior," Journal of Social Issues, $1966, \underline{22}(4), 103-115$.

Haar, Charles M. "Wayne Township: Zoning for whom?--In brief reply," Harvard Law Review, 1954, 67, 986.

- Land Use Planning. Boston: Little, Brown, 1959.

Hall, Edward T. The Hidden Dimension. Garden City: Doubleday, 1966.

Hartman, Chester. "The limitations of public housing: Relocation choices in a working-class community," Journal of the American Institute of Planners, 1963, 29, 283-296. 
Hartman, Chester W. "Social values and housing orientation," Journal of Social Issues, $1963,19(2), 113-131$.

Hays, W.L. Basic Statistics. Belmont, Cal.: Brooks-Cole, 1967.

Hebb, Donald 0. The Organization of Behavior. New York: Wiley, 1949. Saunders Co., $\frac{\text { Textbook }}{1966 .}$ of Psychology, 2nd ed. Philadelphia: W.B. $466-477$.

Holme, Anathea \& Peter Massie. Children's P1ay: A Study of Needs and Opportunities. London: Michael Joseph, Ltd., 1970.

Hu11, Clark L. Principles of Behavior. New York: Appleton, Century, Crofts, 1943.

Husser1, Edmund. Cartesian Meditations, tr. Dorion Cairns. The Hague: Nijhoff, 1960.

Hutt, C. \& M. Vaizey. "Differential effects of group density on social behavior," Nature, 1966, 209, 1371-1372.

Jacobs, Jane. The Death and Life of Great American Cities. New York: Vintage, 1961 .

Jephcot, Pearl. Homes in High Flats. Edinburgh: 01iver \& Boyd, 1971.

Junker, Buford. Field Work. Chicago: University of Chicago Press, 1960.

Kaplan, Abraham. The Conduct of Inquiry. San Francisco: Chandler, 1964.

Kaplan, Stephen, Rachel Kaplan, John Wendt. "Rated preference and complexity for natural and urban visual material," Perception and Psychophysics, $1972,12(4), 354-356$.

Kiefer, Christie W. "Personality and Social Change in a Japanese Danchi," Dissertation Abstracts, 1968, 29, 1246-b.

Keller, Suzanne. The Urban Neighborhood. New York: Random House, 1968.

Keyfitz, Nathan. "Population density and style of social life," Bioscience, $1966,16,868-873$.

Klausner, Samuel Z. On Man and His Environment. San Francisco: JosseyBass, 1971.

Kohler, Wolfgang. Gestalt Psychology. New York: Liveright, 1929. 
Laurendeau, Monique \& Adrien Pinard. The Development of the Concept of Space in the Child. New York: International Universities Press, 1970.

Lawrence, John E.S. "Science and sentiment: Overview of research on crowding and human behavior," Psychological Bulletin, 1974, 81, 712-720.

LeCompte, William F. "Behavior-settings as data-generating units for the environmental planner and architect," in J. Lang, C. Burnette, W. Moleski, D. Vachon, Designing for Human Behavior: Architecture and the Behavioral Sciences. Stroudsburg, Pa.: Dowden, Hutchinson, \& Ross, INc., 1974.

Lee, Terrence. "Urban Neighborhoods as a socio-spatial schema," Human Relations, 1968, 21, 241-267.

Lipman, Marvin. "Housing and environment," Habitat, 1969, 12(2), 2-6.

Lofland, Lyn. A World of Strangers. New York: Basic Books, 1973.

Loo, Chalsa. "Effects of Spatial Density on Social Behavior of Children," Dissertation Abstracts, 1971, 32, 4189-b.

-_- "Important issues in researching the effects of crowding on humans," Representative Research in Social Psychology, 1973, 4, 219-226.

Loring, William C., Jr. "Housing characteristics and social disorganization," Social Problems, 1956, 3, 160-168.

Lowentha1, David. "Geography, experience, and imagination: Toward a geographical epistemology," Annals of Association of American Geographers, $1961,51,241-260$.

Lowenthal, David. "Introduction: Environmental perception and behavior," in D. Lowentha1, Environmental Perception and Behavior. Chicago: University of Chicago, Department of Geography, Research Paper 109, 1967 .

Lynch, Kevin. The Image of the City. Cambridge, Mass.: MIT Press, 1960.

Marsden, Halsey M. "Crowding and animal behavior," in J.F. Wohlwill \& D.H. Carson, eds., Environment and the Social Sciences. Washington D.C.: American Psychological Association, 1972.

Marsha11, Nancy C. Orientations Toward Privacy: Environmental and Personality Components. unpublished $\mathrm{Ph} . \mathrm{D}$. dissertation, University of California at Berkeley, 1969.

Marx, Leo. The Machine in the Garden. New York: Oxford, 1964. 
Maslow, A.H. \& N.L. Mintz. "Effects of aesthetic surroundings. I." Journal of Psychology, 1956, 41, 247-254.

McGuire, Carson \& George D. White. The Measurement of Social Status. Research Paper in Human Development 非3, Department of Educational Psychology, University of Texas, 1955.

Meier, Richard A. A Communication Theory of Urban Growth. Cambridge, Mass.: MIT Press, 1962.

Merleau-Ponty, Maurice. Phenomenology of Perception. London: Routledge \& Kegan Paul, 1962.

Merton, Robert K. "The social psychology of housing," in W. Dennis, ed., Current Trends in Social Psychology. Pittsburgh: University of Pittsburgh Press, 1948.

Milgram, Stanley. "The experience of living in cities," Science, 1970, $167,1461-1468$.

Miller, J. Marshall. "Residential density: Relating people to space rather than to ground area," Journal of the American Institute of Planners, 1961, 27, 77-78.

Mitche11, Robert E. "Some social implications of high density housing," American Sociological Review, 1971, 36, 18-29.

Mumford, Lewis. The City in History. New York: Harcourt, Brace, \& World, 1961.

Natsoulas, Thomas. "The subjective, experiential element in perception," Psychological Bulletin, 1974, 81, 611-631.

Papanek, Victor. Design for the Real World. New York: Pantheon, 1971.

Parr, A.E. "Environmental design and psychology," Landscape, 1964, 14(2), 15-18.

-Psychological aspects of urbanology," Journal of Social Issues, $1966,22(4), 39-45$.

Piaget, Jean \& Barbel Inhelder. The Child's Conception of Space. New York: Norton, 1967.

Pillorge, George. "Intensity of Development and Livability of MultiFamily Housing Projects: a Review" Journal of the American Institute of Planners, 1964, 30, 257-259.

Plant, J.S. "Family living space and personality development," in N.W. Bell \& E.F. Vogel, eds., A Modern Introduction to the Family. New York: Macmillan, 1960. 
Plattner, Stuart. "Rural market networks," Scientific American, 1975, $232(5), 66-79$.

Rapoport, Amos. House Form and Culture. Englewood Cliffs: PrenticeHa11, 1969.

Riemer, Svend. "Sociological theory of home adjustment," American Sociological Review, 1943, 8, 272-278.

Robbins, Michae1 C. "Perceptual environment and pattern-preferences," Perceptual and Motor Skills, 1968, 26, 545-546.

Rosow, I. "The social effects of the physical environment," Journal of the American Institute of P1anners, 1961, 27, 127-133.

Schatzman, Leonard \& Anselm L. Strauss. Field Research: Strategies for a Natural Sociology. Englewood-Cliffs: Prentice-Ha11, 1973.

Schmitt, Robert C. "Density, health, and social disorganization," Journal of the American Institute of Planners, 1966, 32, 38-40.

Schooler, Carmi. "Social antecedents of adult psychological functioning," American Journal of Sociology, 1972, 78, 299-322.

Schorr, Alvin C. Slums and Social Insecurity. Washington, D.C.: U.S. Government Printing Office, 1963.

Simmel, Georg. "The Metropolis and Mental Life," in The Sociology of Georg Simnel, K. Wolff, ed., Glencoe: Free Press, 1950.

Smith, Dorothy E. "Household space and family organization," Pacific Sociological Review, 1971, 14, 53-78.

Sommer, Robert. "Man's proximate environment," Journal of Social Issues, $1966,22(4), 59-70$.

Sonnenfeld, Joseph. "Variable values in space and landscape: an inquiry into the nature of environmental necessity," Journal of Social Issues, $1966, \underline{22(4), 71-82 .}$

Stevenson, Anne, Elaine Martin, Judith o'Nei11. High Living: A Study of Family Life in Flats. Melbourne: Melbourne University, 1967.

Stokols, Daniel. "A social-psychological model of human crowding phenomena," Journal of the American Institute of Planners, 1972, 38, $72-83$.

Studer, Raymond \& David Stea. "Architectural programming and human behavior," Journal of Social Issues, 1966, 22(4), 127-136.

Terrien, Frederic W. \& Donald L. Mil1s. "The effects of changing size upon the internal structure of organizations," American Sociologica1 Revièw, 1955, 20, 11-13. 
U.S. Bureau of Census. Census of Population and Housing: 1970 Block Statistics. Washington, D.C.: U.S. Government Printing office, 1971.

- Census of Population and Housing: 1970 Census Tracts. Washington, D.C.: U.S. Givernment Printing office, 1972.

Wade, Glenn R. A Study of Free Play Patterns of Elementary School Age Children on Playground Equipment. unpublished Master's thesis, The Pennsylvania State University, 1968.

Wells, Brian W.P. "The psycho-social influence of building environment: Sociometric findings in large and small office spaces," Building Science, $1965, \underline{1}, 153-165$.

Werner, Heinz. Comparative Psychology of Mental Development. Chicago: Follet, 1948 .

White, L.E. "The outdoor play of children living in flats," in L. Kuper, ed., Living in Towns. London: Cresset Press, 1953.

White, Morton \& Lucia White. The Intellectual Versus the City. New York: Mentor, 1962.

Willmott, Peter \& Michael Young. Family and Class in a Londor Suburb. London: Routledge \& Kegan Paul, 1960.

Wilner, Daniel M., Rosabelle Walkley, Thomas Pinkerton, Matthew Tayback. The Housing Environment and Family Life. Baltimore: John Hopkins, 1962 .

Wilson, Granville. "British blame rise in youth crime rates on greedy, indulgent, affluent parents," The Oregonian, September 29, 1974, p. 3-E.

Wines, James. "Notes from a passing car: The problem of art in a mobile environment," Architectural Forum, 1973, 139(2), 66-75.

Winsborough, Halliman $\mathrm{H}$. "The social conseçuences of high population density," Law and Contemporary Problens, 1965, 30, 120-126.

Winter, Mary. Apartment and Single Family Dwellings as They Affect the Play Activities of Pre-School Children. unpublished Master's thesis, The Pennsylvania State University, 1966.

Wirth, Louis W. "Urbanism as a way of life," American Journal of Sociology, 1938, 44, 1-24.

Witkin, H.A., A.R. Goodenough, S.A. Kays. "Stability of cognitive style from childhood tc young adulthood," Journal of Personality and Social Psychology, 1967, 7, 291-300. 
Wohlwi1l, Joachim. The Study of Behavioral Development. New York: Academic Press, 1973.

Wolpert, Julian. "Migration as an adjustment to environmental stress," Journal of Social Is sues, $1966,22(4), 92-102$.

Woodworth, Robert S. Psychology, Rev. Ed. New York: Holt, Rinehart \& • Winston, 1929.

Yancey, W.L. "Architecture, interaction, and social control," in J.F. Wohlwill \& D.Carson, eds., Environment and the Social Sciences. Washington, D.C.: American Psychological Association, 1972.

Zlutnik, Steven \& Irwin Altman. "Crowding and human behavior," in J.F. Wohlwill \& D. Carson, eds., Environment and the Social Sciences. Washington, D.C.: American Psychological Association, 1972. 
APPENDICES

APPENDIX A. First Letter to Parents

APPENDIX B. Second Letter to Parents

APPENDIX C. Interview Checklist

APPENDIX D. Instructions for Coding Protocols

APPENDIX E. McGuire-White Scale 
Appendix A

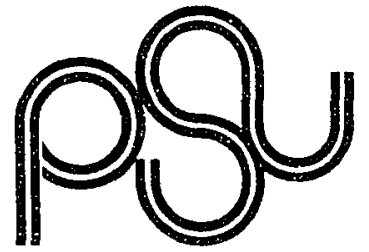

13 January 1975

2

Dear Parent:

I am writing to ask you and your child to take part in a study I am doing.

I am getting my Doctorate in Urban Studies, and for my dissertation I want to look at how children adjust to living in apartments or singlefamily homes. Unlike the larger Eastern cities, Portland has few apartment areas where there are also children; so I have come to the Helen Gordon Center, where there are children, many of whom live in apartments scattered all over the city.

I w11l use as little of your time as is possible--certainly no more than half an hour. I want to ask you a few questions about what you do for relaxation, and I will then ask your child similar questions. Since I would prefer that your answers be spontaneous and uncontaminated by my expectations, I'd rather not go into specifics about my study until after I've asked my questions; but then I'd be more than happy to talk to you about it. When the study is completed, I'1l be sending out a letter describing the results.

The study will be written in such a way that no child can be identified; I will not even be using family names in my own records. I will try to arrange my interviewing schedule to be as convenient for you as is possible.

Un1ess I hear otherwise, I will assume you are willing to participate in the study and in a week I will be contacting you to arrange a time--late afternoons on weekdays, or on the weekend--to come and ask my questions.

If you have any questions, now or at any time in the study, I have an office on campus in room 2, Francis Manor (229-4056); my home phone number is 228-2919.

Sincerely, 
Appendix B

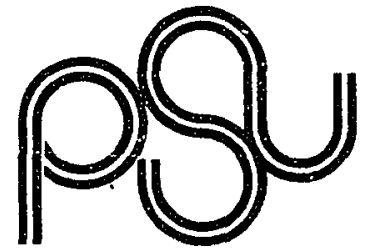

2 April 1975

UNIVERSITY

p.o. box 751

portland, oregon

97207

$503: 229-40 \div 3$

Urban Studies

graduate program

\section{Dear Parents:}

My research on the adjustment of children to apartments or single-family homes is at a point that I need to talk to you about it. I had planned to do this earlier in the research, but my committee felt it would be better to wait until now. I would like to arrange a convenient time for me to visit.

The visit will be short, certainly no more than half an hour. I will want to ask some basic descriptive questions--number of rooms in the home, number of children, etc. I will also want to ask a few questions about how you spend spare time, and how your child spends it. Then I will want to ask your child how he/she spends spare time. It is important that I ask your child these questions at home, since "being there" will probably serve as a memory-aid for him/her.

After I have asked my questions, I would be glad to answer any of yours. In fact, after the study is over and I've looked at the results, I would be glad to discuss the entire study with as many of you parents as are interested.

I expect that the best times to visit would be from 3:00--7:00 on weekdays, and 9:00 AM--7:00 PM on weekends. I would like to begin the visits this weekend (April $5 \& 6$ ). I will be telephoning you to set up an appointment, or if there is a time that is particularly convenient you could contact me. My office is room 2, Francis Manor. My office phone is 229-4056; my home phone is 228-2919.

I don't wish to inconvenience anyone; at the same time, I'd prefer to complete the visits before the term is too far along and either you or I get bogged down with work. I would like to emphasize that I am available for these appointments at whatever time is most convenient for you.

$$
\text { Sincerely, }
$$

Anthony J. Filipovitch 
Appendix C

INTARVIEN CHECEIIST

\begin{tabular}{|c|c|c|}
\hline CODes: & NAWS: & $\begin{array}{l}\text { Mos, at HGE : } \\
\text { DNTS: }\end{array}$ \\
\hline
\end{tabular}

1. Paystexl Disonsion of housins Intioneont

- a. Number of units in butlding (20-21)

1) Inside staimay? (22)

- 11) Untts por belliay (23-24)

- 111) Number of floors in structure (25-26)

- b. sumier of rooms in unit (27-28)

- - _c. Size of unit $(29-32)$

2. Socio-Spotial Ditension of Housing Environment

- - a. Murber of poopio in bullding (33-35)

- - b. Numior of poors in butloling $(36-37)$

- c. Nivber of poers in nolghbornood (39-39)

- - d. Tros Ifvod in busiding (40-41)

- - . Itoo 1100d in butlding-typo $(42-43)$

3. Soc1sl Dimonsion of Housing Bnvinonmont
- Aurber of childron in hoso (44)
- b. Number or adults carling for chlld (45)
- c. Parant accupation (45)
- d. Grandperants occupation, ir perents aro sturionts (47)
- o. SES (48)

4. Chlld's Pley (Parents)

- a. Percont of time in solitary or parallel play (49-50)

- - b. Number of pars pleyod with $(51-52)$

- - _c. Where does your child usurlly pleg? (53-56) (Cospato area)

-

5. Dosor's Test (Paronts)

"Imegine this is a room ware poople rolex togather. Aut as many poople in It a you cen whout mating it crowd."

$-(57-58)$

6. Child's Play (Child)

- 2. mpto do you play with? fisoso co thoy 137e?" (59-50)

- b. "Bore do you usually pieg?" (61-62)

7. Dosor's Test ( $\mathrm{Cbild}$ )

"Frotend this is a room were childron aro playing. And protond thet these aro chlidran. Put som of thoso chtlizen in the room so thay aro playing. but don"t use so men that thoy ary uncomfariablo and eroudad togother." $(6 j-64)$ 


\section{APPENDIX D}

\section{INSTRUCTIONS FOR CODING PROTOCOLS}

"Behavior episode" is the name given to any unit of the stream of behavior; it is what someone would tell you he is doing, if you asked him. The coding will be devoted to determining the exact bounds of behavior episodes. You will be given a set of protocols which are intended to cnntain one behavior episode, although some actions may be included at the beginning or at the end, or hoth, of a protocol that are not part of the episode.

Read each protocol through. Decide what the goal of the behavior appears to be. Then, re-read the protocol, examining each action in turn. To be included in the behavior episode, each action must conform to the three guidelines listed below. Once a behavior episode begins, it is ended when the flow of the episode is terminated (by success of failure) or interrupted.

\section{Guidelines for Determining Behavior Episodes}

I. Constancy of Direction--Does the action fall within the flow of the episode?

A. Criteria:

1. Continuation of earlier action

2. Renewal of earlier action after forced digression

3. Action preparing for attainment of goal

4. Sustained action suddenly ceases when goal is reached

5. Action articulated with all others (i.e., different actions, but continuity of flow)

6. Action following shortest path to goal (e.g., turning away from goal to get around an obstacle)

7. Action, while varied, parallels movement in setting 
B. Ambiguity

When in doubt, code for a longer episode (In absence of positive indication of change, continue).

II. Normal Behavior Perspective--Can the person perceive it as anit?

A. Criteria

1. Change in behavior perspective generally parallels change in direction.

2. Determined by intended terminal position

B. Ambiguity

When doubt, code for the shorter episode (In absence of positive indicators to the contrary, stop).

III. Superior Whole Potency--Does the goal of the episode absorb more attention than its parts?

A. Criteria

1. If an action has more than one goal, at least one subgoal must flow in the same direction as the whole episode.

2. If an action has only one goal and that goal is not in the same direction as the whole episode, that action, no matter how brief, terminates the episode.

B. Ambiguity

When in doubt, code for a longer episode (In absence of positive indication of change, continue). 


\section{APPENDIX E}

THE MCGUIRE-WHITE SCALE FOR THE MEASUREMENT OF SOCIAL STATUS*

The McGuire-Thite Scale for the Measurement of Social Status is a three-part index, composed of scales for Education, Occupation, and Source of Income. The scales are similar to those used by warner, updated and simplified. The scales were used in this study merely to provide a simplified system for ranking families; no attempt was made to translate the ranks into a specific socio-economic level. Accordingly, the scales could be used independently. The Education Scale was not used, since almost all the parents were currently enrolled in the University.

SOURCE OF INCOME SCALE

The categories of the Source of Income Scale include:

1. Inherited Savings and investments; "old money."

2. Earned wealth; "new money."

3. Profits, fees royalties; executives with profit sharing.

4. Salary, comission, regular monthly or yearly income.

5. Hourly wages, piecework.

6. Odd-jobs, private relief

7. Public relief or charity

*Carson McGuire \& George D. White, The Measurement of Social Status, Research Paper in Human Development "3, Department of Educational Psychology, University of Texas, 1955. 
Professional Propriotors Businesgmen White Collax

Blue Collar Service

1. Lawyer, Judge, Large business Top bxecs. CPA, Newspaphysictan, valued over of Corps., per editor professor $\$ 100,000$ Banks, otc.

2. Is teacher, Business Managers, Accountant, other over valued at Supervisors Insurance, 4 yr. degreo \$50-100 T Supervisors Insurance,
Real Estate

3. GS teacher, Business other with valued at 4 -year degreo $\$ 10-50 \mathrm{~T}$

4.

Branch Hgr. Bank \&

Salesman of Postal clerks Brand goods

Stenogra pher

Salesperson

Clerk in

grocery or

varlety store

6.

7. Reputed Lawbreakers

\begin{tabular}{|c|c|}
\hline BLue Colvar & Sorvice \\
\hline \multicolumn{2}{|l|}{$\begin{array}{l}\text { Sma II } \\
\text { contractor }\end{array}$} \\
\hline $\begin{array}{l}\text { Foreman, } \\
\text { Haster crafts- } \\
\text { man }\end{array}$ & $\begin{array}{l}\text { Police } \\
\text { - Captain }\end{array}$ \\
\hline $\begin{array}{l}\text { Apprentice, } \\
\text { Skilled } \\
\text { Labor }\end{array}$ & Policeman \\
\hline $\begin{array}{l}\text { Seml-skilled } \\
\text { labor }\end{array}$ & $\begin{array}{l}\text { taxidriver, } \\
\text { waitress }\end{array}$ \\
\hline $\begin{array}{l}\text { Unsktlled } \\
\text { labor } \\
\text {. }\end{array}$ & $\begin{array}{l}\text { Domestio \& } \\
\text { kitchen } \\
\text { help }\end{array}$ \\
\hline
\end{tabular}

